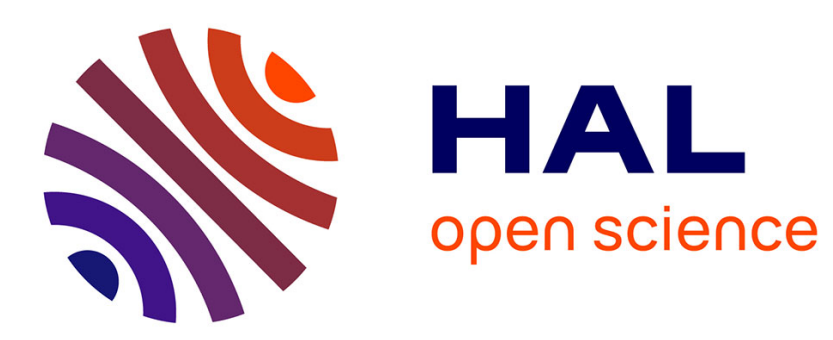

\title{
Passive and Self-propelled Locomotion of an Elastic Swimmer in a Perfect Fluid
}

\author{
Alexandre Munnier
}

\section{To cite this version:}

Alexandre Munnier. Passive and Self-propelled Locomotion of an Elastic Swimmer in a Perfect Fluid. SIAM Journal on Applied Dynamical Systems, 2011, 10 (4), pp.1363-1403. 10.1137/100805455 . hal-00509657v2

\author{
HAL Id: hal-00509657 \\ https://hal.science/hal-00509657v2
}

Submitted on 31 Aug 2010

HAL is a multi-disciplinary open access archive for the deposit and dissemination of scientific research documents, whether they are published or not. The documents may come from teaching and research institutions in France or abroad, or from public or private research centers.
L'archive ouverte pluridisciplinaire HAL, est destinée au dépôt et à la diffusion de documents scientifiques de niveau recherche, publiés ou non, émanant des établissements d'enseignement et de recherche français ou étrangers, des laboratoires publics ou privés. 


\title{
PASSIVE AND SELF-PROPELLED LOCOMOTION OF AN ELASTIC SWIMMER IN A PERFECT FLUID
}

\author{
ALEXANDRE MUNNIER*
}

\begin{abstract}
In this paper we are interested in studying the free motion of a hyperelastic body (also called swimmer) immersed in a perfect fluid. We derive the Euler-Lagrange equations from the Least Action Principle of Lagrangian Mechanics and prove that they are well-posed when the number of elastic modes is finite. The recourse to a strain energy density function in the modeling allows many different constitutive equations for the hyperelastic material to be considered. We perform numerical simulations, aiming to study passive locomotion (i.e. locomotion at zero energy cost). As a first quite surprising result, we observe that the swimmer does not even have to be elastic to experience passive locomotion in its idealized environment. Indeed, we provide an example of deformable (but non elastic) swimmer, for which the fluid-body system behaves as an oscillating mechanical system. The shape changes caused solely by the hydrodynamical forces on the body's boundary turn out to be periodic strokes resulting in locomotion. This phenomenon can be seen as a generalization, to deformable bodies, of the famous D'Alembert's paradox (1752) [11], claiming that the drag force is zero on a rigid solid moving with constant velocity. Many other examples of passive locomotion, involving different types of hyperelastic swimmers, are studied. A special interest is devoted to the study of energy and impulse exchanges between the fluid and the body.

In the last section, we assume that the swimmer has the ability to modify its shape by means of internal forces. We prove that in this case, the equations of motion are still well-posed and we illustrate again with numerical simulations that, starting from rest, self-propelled locomotion can be achieved.
\end{abstract}

Key words. Passive locomotion, elastic swimmer, perfect fluid, D'Alembert's paradox.

AMS subject classifications. 74F10, 76Z10, 73C20, 76B40.

1. Introduction. The study of the self-propelled locomotion in a fluid by shape changes has been drawing the attention of mathematicians for a decade. Although, at first, most of these works have mainly aimed to improve the academic understanding of the locomotion of fish and aquatic mammals, a challenging application emerged and sharpened the interest of scientists: the design of biomimetic robots. Indeed, such swimming devices propelled and steered by shape changes would be more efficient, stealthier and more maneuverable than if propeller-driven.

From the pioneering works of Galdi [12, 13], many mathematical models have been proposed. Besides the modeling, authors have been mainly concerned with the well-posedness of the equations of motion. Let us mention [15] in which the authors consider the dynamics of an articulated body in a perfect potential fluid and [26] which provides a model for a fish-like swimming body in a viscous fluid. We refer to [21, 22] and [23] for a more comprehensive bibliography on this topic. It is worth noticing that in all of these articles, the deformations of the swimmer do not result from the interaction with the surrounded fluid but are prescribed as functions of time. It is also the case in [23] and [7], although in these papers the authors prove that there is a one-to-one correspondence between the shape changes and the internal forces, within the body, they are caused by.

Although crucial for the design of autonomous underwater vehicles, theoretical results on controllability or motion-planning are rare so far. Actually, only two types of fluid are simple enough to allow controllability results: very viscous fluids driven by steady-state Stokes equations and perfect fluids with potential flow. Furthermore, most of the published results in this field concern so-called articulated bodies as in the works of Alouges, DeSimone and Lefebvre [1] (dealing with a threesphere mechanism swimming in a high Reynolds number fluid) or those of Melli, Rowley and Rufat

*Institut Elie Cartan UMR 7502, Nancy-Université, CNRS, INRIA, B.P. 239, F-54506 Vandoeuvre-lès-Nancy Cedex, France (alexandre.munnier@iecn.u-nancy.fr), and INRIA Lorraine, Projet CORIDA. 
[20]. We deal with a more complex model in [7], where we prove the approximated controllability of a amoeba-like swimming body in a perfect fluid.

These models, to be more realistic, would require being supplemented with a dynamic for the elasticity of the body. The main reason this has not been done before is that coupling the equations of Fluid Dynamics (usually written within the Eulerian framework) to the equations of elasticity (expressed within the Lagrangian formalism) is a difficult task. The existence of solutions for such a coupled system of equations has been investigated quite recently in a series of papers $[2,5,10,3,8]$. In all of these articles, the fluid is assumed to be viscous and incompressible; the flow being governed by Navier-Stokes equations.

In this paper, we propose a 2D model for the dynamic interaction between an ideal fluid and a deformable swimmer, which can be elastic or not; the fluid-body system filling the whole space. The model of deformable body we consider is inspired by that of Shapere and Wilczek introduced in [27] and further discussed in [9]. The Euler-Lagrange equations are obtained by applying the least action principle of Lagrangian Mechanics to the overall fluid-body system. This idea goes back to the works of Thomson, Tait and Kirchhoff in their studies of the motion of rigid solids in a perfect fluid, as described in the book of Lamb [17]. The main advantage of this method is to avoid the troublesome calculation of the pressure on the body's surface. Furthermore, since the elastic swimmer and the fluid are treated as forming together one dynamical system, we also avoid the tricky task of writing the dynamic coupling conditions between the equations of Fluid Mechanics, Solid Mechanics and Elasticity taking place on the body's surface.

Our model of elasticity, based on the use of a strain energy density function, is simple enough to allow locomotion properties to be investigated but still allows many different constitutive equations for the hyperelastic material to be considered. We show that the Euler-Lagrange equations of the system can be reduced to a system of ODEs. When the number of elastic modes is finite, the system of ODEs is also finite and we prove that it admits a unique smooth solution, well defined for all time. Then we show through numerical simulations that, by choosing properly the initial conditions for the fluid-body system, passive locomotion is possible. It means than within the isolated fluid-body system (under no external applied forces), the swimmer undergoes elastic deformations resulting in locomotion. This motion is endless because the system is conservative. The displacement is due to the constant exchange of momentum between the shape changes and the fluid motion. A particular example is worth being highlighted, involving a deformable (non elastic) swimmer. Starting with zero initial rigid velocity, the shape changes (caused solely by the hydrodynamical forces on the body's boundary) produce time periodic strokes making the body swim. This phenomenon can be seen as a generalization, to deformable bodies, of the famous D'Alembert's paradox (1752) [11], claiming that the drag force is constantly zero on a rigid solid moving with constant velocity. In our example, the hydrodynamical forces are not zero for all time but turn out to be zero on average, over a stroke. Notice that examples of passive locomotion have already been obtained in [16] with toy models consisting in one-dimensional interconnected springs and mass, submerged in a perfect fluid.

At last, we consider that the swimmer has the ability to modify its shape under the action of internal forces. The Euler Lagrange equations have to be modified accordingly and we prove that they are still well-posed. We perform again numerical simulations to illustrate some selfpropelled locomotion strategies. This is, to our knowledge, the first example of elastic shape changing swimming body.

The paper is organized as follows: in the next Section, we address the modeling of a shape changing swimmer in a perfect fluid. The Euler-Lagrange equations are obtained in Section 3. Sec- 
tion 4 is devoted to the modeling of the elasticity and to the numerical study of passive locomotion. Self-propelled locomotion of the elastic body is investigated in Section 5. The numerics of this paper is supplemented with a web page containing videos and additional material. It is located at http://www.iecn.u-nancy.fr/ munnier/passive_locomotion/index.html.

2. Modeling. Although slightly different, most of the material of this section has already been introduced and detailed in [7]. For the sake of convenience, we will recall the definitions and important results and refer to [7] for the proofs.

The modeling requires considering a physical space and a computational space. Both are identified either with $\mathbf{R}^{2}$ or with the complex field $\mathbf{C}$. To describe $2 \mathrm{D}$ vectors, we will mix the complex notation $z=z_{1}+i z_{2}\left(i^{2}=-1\right)$ with the real one $z=\left(z_{1}, z_{2}\right)^{T}$ or use the polar coordinates $(r, \theta)$ with $z=r e^{i \theta}$ when necessary.

The computational space is endowed with a frame $\left(\mathbf{E}_{1}, \mathbf{E}_{2}\right), D$ stands for the open unit disk and $\Omega:=\mathbf{C} \backslash \bar{D}$.

We introduce two frames in the physical space: a Galilean fixed one $\left(\mathbf{e}_{1}, \mathbf{e}_{2}\right)$ and a moving one $\left(\mathbf{e}_{1}^{*}, \mathbf{e}_{2}^{*}\right)$, the origin of which coincides at any time with the center-of-mass of the elastic body. Therefore, the coordinates of the center-of-mass of the body are $\mathbf{r}:=\left(r_{1}, r_{2}\right)^{T}$ in $\left(\mathbf{e}_{1}, \mathbf{e}_{2}\right)$ and $(0,0)^{T}$ in $\left(\mathbf{e}_{1}^{*}, \mathbf{e}_{2}^{*}\right)$. More generally, the quantities will be denoted with asterisks when expressed in the moving frame: for instance, the domain occupied by the body is $\mathcal{B}$ in $\left(\mathbf{e}_{1}, \mathbf{e}_{2}\right)$ and $\mathcal{B}^{*}$ in $\left(\mathbf{e}_{1}^{*}, \mathbf{e}_{2}^{*}\right)$ while $\mathcal{F}:=\mathbf{R}^{2} \backslash \overline{\mathcal{B}}$ and $\mathcal{F}^{*}:=\mathbf{R}^{2} \backslash \overline{\mathcal{B}}^{*}$ both stand for the domain of the fluid.

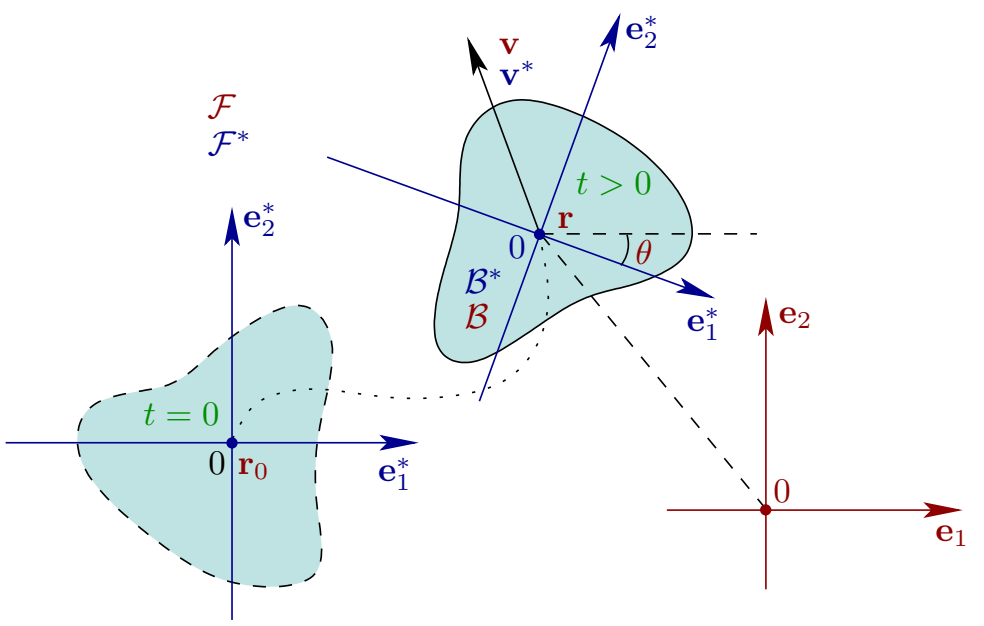

FIG. 2.1. The frame $\left(\mathbf{e}_{1}, \mathbf{e}_{2}\right)$ is fixed and Galilean and the frame $\left(\mathbf{e}_{1}^{*}, \mathbf{e}_{2}^{*}\right)$ is attached to the swimmer and moves along with it. The moving frame is centered at the body's center of mass, the coordinates of which are $\mathbf{r}=\left(r_{1}, r_{2}\right)^{T}$ in the Galilean frame. Quantities are denoted with an asterisk when they are expressed in the moving frame $\left(\mathbf{e}_{1}^{*}, \mathbf{e}_{2}^{*}\right)$.

2.1. Shape changes. Let $\mathcal{S}$ be the Banach space consisting of the complex sequences ${ }^{1} \mathbf{c}:=$ $\left(c_{k}\right)_{k \leq 1}, c_{k}=a_{k}+i b_{k}, a_{k}, b_{k} \in \mathbf{R}$ such that $c_{0}=0$ and $\|\mathbf{c}\|_{\mathcal{S}}:=\sum_{k \leq 1}|k|\left(\left|a_{k}\right|+\left|b_{k}\right|\right)<+\infty$. We will require also the classical Hilbert space $\ell^{2}(\mathbf{C})$, the norm of which is $\|\mathbf{c}\|_{\ell^{2}(\mathbf{C})}:=\sqrt{\sum_{k \leq 1}\left|c_{k}\right|^{2}}$.

\footnotetext{
${ }^{1}$ The indices of the sequences range from $-\infty$ to 1 . It may be surprising but will be justified later on.
} 
The shape changes of the body are described by means of a set of $\mathcal{C}^{1}$ functions $\chi(\mathbf{c}): \bar{D} \rightarrow \overline{\mathcal{B}}^{*}$ depending on the parameter $\mathbf{c} \in \mathcal{S}$ (c will be called in the sequel the shape variable) and defined in complex notation by:

$$
\chi(\mathbf{c})(z):=c_{1} z+\sum_{k \geq 1} c_{-k} \bar{z}^{k}, \quad(z \in \bar{D}),
$$

where $\bar{z}=z_{1}-i z_{2}$ is the complex conjugate of $z=z_{1}+i z_{2}$. Since the domain of the body is defined by $\overline{\mathcal{B}}^{*}:=\chi(\mathbf{c})(\bar{D})$ and even though it does not appear in the notation, $\mathcal{B}^{*}$ depends on the shape variable. The shape variable lives in:

$$
\mathcal{D}:=\left\{\mathbf{c}:=\left(c_{k}\right)_{k \leq 1} \in \mathcal{S}: \sup _{z \in \partial D}\left|\sum_{k \geq 1} k c_{-k} z^{k}\right|<\left|c_{1}\right|\right\},
$$

which is an open subset of $\mathcal{S}$, an infinite dimensional Banach space, so the number of degrees of freedom corresponding to the body's deformations is also infinite. We next introduce the function $\phi(\mathbf{c})$ that maps $\bar{\Omega}$ onto $\overline{\mathcal{F}}^{*}$. It is defined for all $\mathbf{c} \in \mathcal{D}$ by:

$$
\phi(\mathbf{c})(z):=c_{1} z+\sum_{k \leq-1} c_{k} z^{k}, \quad(z \in \bar{\Omega}) .
$$

Since $\bar{z}=1 / z$ for all $z \in \partial D$, we deduce that $\left.\chi(\mathbf{c})\right|_{\partial D}=\left.\phi(\mathbf{c})\right|_{\partial \Omega}$ and the map defined below is continuous in $\mathbf{C}$ for any $\mathbf{c} \in \mathcal{D}$ :

$$
\Phi(\mathbf{c})(z):= \begin{cases}\chi(\mathbf{c})(z) & \text { if } z \in D, \\ \phi(\mathbf{c})(z) & \text { if } z \in \bar{\Omega} .\end{cases}
$$

The main properties of $\chi, \phi$ and $\Phi$ are summarized in the following proposition whose the proof is given in [7]:

Proposition 2.1. For all $\mathbf{c} \in \mathcal{D}, \chi(\mathbf{c}): \bar{D} \rightarrow \overline{\mathcal{B}}^{*}$ and $\phi(\mathbf{c}): \bar{\Omega} \rightarrow \overline{\mathcal{F}}^{*}$ are both well-defined (the series in (2.1) and (2.2) converge for all $z$ ) and invertible. Besides, $\left.\chi(\mathbf{c})\right|_{D}$ is a $\mathcal{C}^{1}$ diffeomorphism, $\left.\phi(\mathbf{c})\right|_{\Omega}$ is a conformal mapping and $\Phi(\mathbf{c})$ is an homeomorphism from $\mathbf{C}$ onto $\mathbf{C}$.

The main interest in using the shape variable is that we can now describe the shape changes by means of a smooth function of time $t \in[0, T] \mapsto \mathbf{c}(t) \in \mathcal{D}(T>0)$. We denote $\dot{\mathbf{c}}=\left(\dot{c}_{k}\right)_{k \leq 1}$ its time derivative defined for all $t \in[0, T]$, with $\dot{c}_{k}:=\dot{a}_{k}+i \dot{b}_{k}(k \leq 1)$. We denote also $\dot{\chi}(\mathbf{c})$ the time derivative of $\chi(\mathbf{c})$ i.e., the mapping $z \in \bar{D} \mapsto \dot{c}_{1} z+\sum_{k \geq 1} \dot{c}_{-k} \bar{z}^{k} \in \mathbf{C}$.

\subsection{Physical quantities.}

2.2.1. Volume, mass and inertia momentum. From the relation $x^{*}:=\chi(\mathbf{c})(z),(z \in D)$, we deduce that the area elements $\mathrm{d} x^{*}$ and $\mathrm{d} z$ of respectively $\mathcal{B}^{*}$ and $D$ can be deduced one from the other by the identity:

$$
\mathrm{d} x^{*}:=J(\mathbf{c})(z) \mathrm{d} z, \quad\left(z \in D, x^{*}:=\chi(\mathbf{c})(z)\right),
$$

where $J(\mathbf{c})(z):=|\operatorname{det} \mathbb{F}(\mathbf{c})(z)|$ and $\mathbb{F}(\mathbf{c})(z):=D \chi(\mathbf{c})(z)$ is the deformation tensor. It entails that the density $\varrho^{*}$ of the body $\mathcal{B}^{*}$ can be deduced from a given constant density $\varrho_{0}>0$ according to the conservation-of-mass principle:

$$
\varrho^{*}\left(x^{*}\right)=\frac{\varrho_{0}}{J(\mathbf{c})\left(\chi(\mathbf{c})^{-1}\left(x^{*}\right)\right)}, \quad\left(x^{*} \in \mathcal{B}^{*}\right) .
$$


The element of mass in $D$ is then defined by $\mathrm{d} m_{0}:=\varrho_{0} \mathrm{~d} z$, and likewise, $\mathrm{d} m^{*}:=\varrho^{*} \mathrm{~d} x^{*}$ is the element of mass in $\mathcal{B}^{*}$. The volume of the body is given by $\operatorname{Vol}(\mathcal{B})=\int_{D} J(\mathbf{c})(z) \mathrm{d} z$. We can establish first (we refer again to [7] for details) that for all $\mathbf{c} \in \mathcal{D}$ :

$$
J(\mathbf{c})(z)=\left|c_{1}\right|^{2}-\left|\sum_{k \geq 0}(k+1) c_{-k-1} \bar{z}^{k}\right|^{2}, \quad(z \in \bar{D}),
$$

and next derive the expression of the volume of the body in terms of the shape variable:

$$
\operatorname{Vol}(\mathcal{B})=\pi\left(\sum_{k \leq 1} k\left|c_{k}\right|^{2}\right)
$$

Due to the incompressibility, the volume of the fluid has to remain constant. We draw the same conclusion for the volume of the swimmer because it is nothing but the complementary of the volume of the fluid. According to (2.5), it means that the function $t \in[0, T] \mapsto\left|c_{1}(t)\right|^{2}-\sum_{k \geq 1} k\left|c_{-k}(t)\right|^{2}$ has to be constant. This observation leads us to introduce for all $\mu \in \mathbf{R}_{+}$, the closed subsets of $\mathcal{S}$ :

$$
\mathcal{A}(\mu):=\left\{\mathbf{c} \in \mathcal{S}: \sum_{k \leq 1} k\left|c_{k}\right|^{2}=\mu^{2}\right\}
$$

Remember that a sufficient condition for the map $\chi(\mathbf{c})$ to be injective is that $\mathbf{c}$ belongs to $\mathcal{D}$. We define then also:

$$
\mathcal{A}^{\bullet}(\mu):=\mathcal{A}(\mu) \cap \mathcal{D}, \quad\left(\mu \in \mathbf{R}_{+}\right) .
$$

Differentiating with respect to time identity (2.5), we get an equivalent formulation for the conservation of the body's volume:

$$
\sum_{k \leq 1} k\left(\dot{a}_{k} a_{k}+\dot{b}_{k} b_{k}\right)=0, \quad(t \geq 0)
$$

The mass of the body is:

$$
m:=\int_{\mathcal{B}^{*}} \mathrm{~d} m^{*}=\int_{D} \mathrm{~d} m_{0}=\pi \varrho_{0} .
$$

Notice that for a neutrally buoyant swimmer, we get the equality $\varrho_{f} \operatorname{Vol}(\mathcal{B})=m$ where $\varrho_{f}>0$ is the given constant density of the fluid. In this case, the densities $\varrho_{f}$ and $\varrho_{0}$ would be linked through the relation:

$$
\varrho_{f} \mu^{2}=\varrho_{0}
$$

The inertia momentum depends on the shape of the body and reads $I(\mathbf{c}):=\int_{\mathcal{B}^{*}}\left|x^{*}\right|^{2} \mathrm{~d} m^{*}$, or equivalently upon a change of variables $I(\mathbf{c}):=\int_{D}|\chi(\mathbf{c})(z)|^{2} \mathrm{~d} m_{0}$. It can also be easily computed in terms of the shape variable:

$$
I(\mathbf{c})=\pi \varrho_{0}\left(\sum_{k \leq 1} \frac{\left|c_{k}\right|^{2}}{|k|+1}\right) .
$$


2.2.2. Physically allowable shape changes. The motion of the swimmer in the fluid is the superimposition of the shape changes and a rigid motion. To obtain a proper decomposition, we impose on the shape changes to let unchanged the linear and the angular momenta of the body with respect to its attached frame $\left(\mathbf{e}_{1}^{*}, \mathbf{e}_{2}^{*}\right)$. For the linear momentum, this condition reads:

$$
\frac{d}{d t}\left(\int_{\mathcal{B}^{*}} x^{*} \mathrm{~d} m^{*}\right)=\mathbf{0}, \quad(t \geq 0),
$$

which can be rewritten, upon a change of variables and taking into account (2.3):

$$
\int_{D} \dot{\chi}(\mathbf{c}) \mathrm{d} m_{0}=\mathbf{0}, \quad(t \geq 0)
$$

For the angular momentum, we introduce for any $x:=\left(x_{1}, x_{2}\right)^{T} \in \mathbf{R}^{2}$ the notation $x^{\perp}=\left(-x_{2}, x_{1}\right)^{T}$ and the same arguments yield:

$$
\int_{D} \dot{\chi}(\mathbf{c}) \cdot \chi(\mathbf{c})^{\perp} \mathrm{d} m_{0}=0, \quad(t \geq 0) .
$$

Condition (2.12) is actually intrinsically satisfied for any smooth shape function $t \in[0, T] \mapsto \mathbf{c}(t) \in$ $\mathcal{S}$. Condition (2.13) can be rewritten in terms of the shape variable as:

$$
\sum_{k \leq 1} \frac{1}{|k|+1}\left(\dot{b}_{k} a_{k}-\dot{a}_{k} b_{k}\right)=0, \quad(t \geq 0) .
$$

This constraint together with (2.8) lead us to introduce the notion of allowable shape variable:

Definition 2.2 (Physically allowable shape variable). A continuously differentiable function $t \in[0, T] \mapsto \mathbf{c}(t) \in \mathcal{S}$ (for some real positive number $T$ ) is said to be physically allowable when:

1. There exists $\mu>0$ such that $\mathbf{c}(t) \in \mathcal{A}^{\bullet}(\mu)$ for all $t \geq 0$.

2. Constraint (2.14) is satisfied for all $t \in] 0, T[$.

2.3. Rigid Motion, Velocity. The (unknown) rigid motion of the swimmer is described by elements $\mathbf{q}:=(\mathbf{r}, \theta)^{T}$ of $\mathcal{Q}:=\mathbf{R}^{2} \times \mathbf{R} / 2 \pi$ where $\mathbf{r}:=\left(r_{1}, r_{2}\right)^{T} \in \mathbf{R}^{2}$ is a vector giving the position of the center-of-mass of the body and $\theta \in \mathbf{R} / 2 \pi$ an angle giving its orientation with respect to $\left(\mathbf{e}_{1}, \mathbf{e}_{2}\right)$ (see Fig. 2.1). Denoting by $R(\theta) \in \mathrm{SO}(2)$ the rotation matrix of angle $\theta$, we get the relations $R(\theta) \mathbf{e}_{j}=\mathbf{e}_{j}^{*}(j=1,2)$.

Let the shape changes be frozen for a while and consider a physical point attached to the body undergoing only a rigid motion. Then, there exists a smooth function $t \in[0, T] \mapsto \mathbf{q}(t):=$ $(\mathbf{r}(t), \theta(t)) \in \mathcal{Q}(T>0)$ such that the coordinates of the point in $\left(\mathbf{e}_{1}, \mathbf{e}_{2}\right)$ be given by $x=R(\theta) x_{0}+\mathbf{r}$ $\left(x_{0} \in \mathbf{R}^{2}\right.$ being the coordinates at the time $\left.t=0\right)$. Next, compute the time derivative of this expression and denote by $\dot{\mathbf{q}}:=(\dot{\mathbf{r}}, \omega) \in \mathbf{R}^{3}$ the time derivative of $\mathbf{q}$. From the classical identity $\partial_{\theta} R(\theta) R(\theta)^{T} x=x^{\perp}$ for all $x=\left(x_{1}, x_{2}\right)^{T} \in \mathbf{R}^{2}$, we deduce that the Eulerian velocity of the point is $\mathbf{v}_{r}(x)=\omega(x-\mathbf{r})^{\perp}+\dot{\mathbf{r}}$. It can also be expressed in the moving frame $\left(\mathbf{e}_{1}^{*}, \mathbf{e}_{2}^{*}\right)$ and $\operatorname{reads} \mathbf{v}_{r}^{*}\left(x^{*}\right)=$ $\omega\left(x^{*}\right)^{\perp}+\dot{\mathbf{r}}^{*}$ where $\dot{\mathbf{r}}^{*}:=R(\theta)^{T} \dot{\mathbf{r}}$. This leads us to introduce also the notation $\dot{\mathbf{q}}^{*}:=\left(\dot{\mathbf{r}}^{*}, \omega\right)^{T} \in \mathbf{R}^{3}$.

Let us go back to the general case where the shape changes are taken into account. The coordinates in $\left(\mathbf{e}_{1}, \mathbf{e}_{2}\right)$ of a physical point attached to the swimmer are given by: $x=R(\theta) \chi(\mathbf{c})\left(z_{0}\right)+$ $\mathbf{r}$, where at the time $t=0, \mathbf{c}(0)=\mathbf{c}_{0} \in \mathcal{D}$ and $x(0)=x_{0}=\chi\left(\mathbf{c}_{0}\right)\left(z_{0}\right)$ for some $z_{0} \in D$. Observe that we can always assume that at the time $t=0, \mathbf{q}=\mathbf{q}_{0}:=(\mathbf{0}, 0)^{T}$. We deduce that the Eulerian velocity at a point $x$ of $\mathcal{B}$ is equal to:

$$
\left.\mathbf{v}(x)=\omega(x-\mathbf{r})^{\perp}+\dot{\mathbf{r}}+R(\theta) \dot{\chi}(\mathbf{c})\left[\chi(\mathbf{c})^{-1}\left(R(\theta)^{T}(x-\mathbf{r})\right)\right)\right] .
$$


When expressed in the moving frame $\left(\mathbf{e}_{1}^{*}, \mathbf{e}_{2}^{*}\right)$ it reads:

$$
\mathbf{v}^{*}\left(x^{*}\right)=\left(\omega x^{* \perp}+\dot{\mathbf{r}}^{*}\right)+\dot{\chi}(\mathbf{c})\left(\chi(\mathbf{c})^{-1}\left(x^{*}\right)\right), \quad\left(x^{*} \in \mathcal{B}^{*}\right) .
$$

2.4. Potential flow. The fluid is assumed to be incompressible and inviscid with constant density $\varrho_{f}>0$. Its element of mass is $\mathrm{d} m_{f}^{*}:=\varrho_{f} \mathrm{~d} x^{*}$ in $\mathcal{F}^{*}$ and $\mathrm{d} m_{f}^{0}:=\varrho_{f} \mathrm{~d} z$ in $\Omega$. The Eulerian velocity of the fluid in $\left(\mathbf{e}_{1}^{*}, \mathbf{e}_{2}^{*}\right)$, denoted by $\mathbf{u}^{*}$ is equal to the gradient of a potential function $\varphi$ i.e. $\mathbf{u}^{*}=\nabla \varphi$ in $\mathcal{F}^{*}$. The incompressibility of the fluid entails that $\nabla \cdot \mathbf{u}^{*}=0$ and hence that

$$
\Delta \varphi=0 \text { in } \mathcal{F}^{*} .
$$

The classical non-penetrating or slip boundary condition for inviscid fluids leads to the identity $\mathbf{u}^{*} \cdot n=\mathbf{v}^{*} \cdot n$ on $\partial \mathcal{F}^{*}$ where $n$ stands here and subsequently for the unit normal to $\partial \mathcal{B}^{*}=\partial \mathcal{F}^{*}$ directed toward the interior of $\mathcal{B}^{*}$. This condition yields a Neumann boundary condition for the potential function:

$$
\partial_{n} \varphi=\mathbf{v}^{*} \cdot n \text { on } \partial \mathcal{F}^{*} .
$$

The boundary value problem (2.16) admits a weak (or variational) solution in the weighted Sobolev space $H_{N}^{1}\left(\mathcal{F}^{*}\right):=\left\{u \in \mathcal{D}^{\prime}\left(\mathcal{F}^{*}\right): u /\left[\sqrt{|x|^{2}+1} \log \left(2+|x|^{2}\right)\right] \in L^{2}\left(\mathcal{F}^{*}\right), \partial_{x_{j}} u \in L^{2}\left(\mathcal{F}^{*}\right), j=1,2\right\}$ where $\mathcal{D}^{\prime}\left(\mathcal{F}^{*}\right)$ is the space of the distributions in $\mathcal{F}^{*}$. This solution is unique up to an additive constant.

Notice that the potential function depends on both $\dot{\mathbf{c}}$ (linearly through its boundary data) and $\mathbf{c}$ (non-linearly through the domain $\mathcal{F}^{*}$ ).

2.5. Lagrangian of the fluid-swimmer system. We neglect the gravity, so the Lagrangian function reduces to the kinetic energy of the fluid-swimmer system. Because of relations (2.12) and (2.13), there is a decoupling between the kinetic energy of the body resulting from its rigid motion and those resulting from its shape changes:

$$
K^{b}:=\frac{1}{2} m\left|\dot{\mathbf{r}}^{*}\right|^{2}+\frac{1}{2}|\omega|^{2} I(\mathbf{c})+\frac{1}{2} \int_{\mathcal{B}^{*}}\left|\dot{\chi}(\mathbf{c})\left(\chi(\mathbf{c})^{-1}\left(x^{*}\right)\right)\right|^{2} \mathrm{~d} m^{*},
$$

the last term being the kinetic energy of deformation. It can be further expanded as follows:

$$
\int_{\mathcal{B}^{*}}\left|\dot{\chi}(\mathbf{c})\left(\chi(\mathbf{c})^{-1}\left(x^{*}\right)\right)\right|^{2} \mathrm{~d} m^{*}=\int_{D}|\dot{\chi}(\mathbf{c})(z)|^{2} \mathrm{~d} m_{0}=\pi \varrho_{0} \sum_{k \leq 1} \frac{\left|\dot{c}_{k}\right|^{2}}{|k|+1} .
$$

On the other hand, the kinetic energy of the fluid reads:

$$
K^{f}:=\frac{1}{2} \int_{\mathcal{F}^{*}}\left|\mathbf{u}^{*}\right|^{2} \mathrm{~d} m_{f}^{*}=\frac{1}{2} \int_{\mathcal{F}^{*}}|\nabla \varphi|^{2} \mathrm{~d} m_{f}^{*} .
$$

eventually, the Lagrangian function of the system fluid-body reduces to:

$$
L:=K^{b}+K^{f},
$$

and turns out to be a function of $\left(\dot{\mathbf{q}}^{*}, \mathbf{c}, \dot{\mathbf{c}}\right) \in\left(\mathbf{R}^{2} \times \mathbf{R}\right) \times \mathcal{D} \times \mathcal{S}$. More precisely, for any fixed $\mathbf{c} \in \mathcal{D}, L(\mathbf{c})$ is a quadratic form in $\left(\dot{\mathbf{q}}^{*}, \dot{\mathbf{c}}\right)$. It is worth remarking that it does not depend on $\mathbf{r}$ and $\theta$ because of the symmetry of our model with respect to the position and orientation of the body in the fluid. 


\section{Euler-Lagrange Equations.}

3.1. Elementary potentials. Using the conformal mapping $\phi(\mathbf{c})(\mathbf{c} \in \mathcal{D})$, we define $\xi(z):=$ $\varphi(\phi(\mathbf{c})(z))(z \in \Omega)$ where $\varphi$ is the potential function. The function $\xi$ is harmonic in $\Omega$. Besides, the properties of conformal mappings allow us to write that

$$
K^{f}=\frac{1}{2} \int_{\mathcal{F}^{*}}|\nabla \varphi|^{2} \mathrm{~d} m_{f}=\frac{1}{2} \int_{\Omega}|\nabla \xi|^{2} \mathrm{~d} m_{f}^{0}
$$

So from now on we will refer to $\xi$ as being the potential function in place of $\varphi$.

3.1.1. Expressions of the elementary potentials. We wish now to decompose $\xi$ into a linear combination of elementary potentials, each one being associated with a degree of freedom of the system. To this purpose, we introduce the functions $\xi_{j}^{r}(\mathbf{c})(j=1,2,3)$ and $\xi_{k}^{a}(\mathbf{c}), \xi_{k}^{b}(\mathbf{c})(k \leq 1$, $k \neq 0)$ as being harmonic in $\Omega$ and satisfying the following Neumann boundary conditions:

$$
\begin{aligned}
& \partial_{n} \xi_{1}^{r}(\mathbf{c})(z)=-\Re\left(z \phi^{\prime}(\mathbf{c})(z)\right), \\
& \partial_{n} \xi_{2}^{r}(\mathbf{c})(z)=-\Im\left(z \phi^{\prime}(\mathbf{c})(z)\right), \\
& \partial_{n} \xi_{3}^{r}(\mathbf{c})(z)=-\Im\left(\overline{\phi(\mathbf{c})(z)} z \phi^{\prime}(\mathbf{c})(z)\right), \quad(z \in \partial D),
\end{aligned}
$$

and

$$
\begin{aligned}
& \partial_{n} \xi_{k}^{a}(\mathbf{c})(z)=-\Re\left(z^{k+1} \phi^{\prime}(\mathbf{c})(z)\right)-k a_{-k}, \\
& \partial_{n} \xi_{k}^{b}(\mathbf{c})(z)=-\Im\left(z^{k+1} \phi^{\prime}(\mathbf{c})(z)\right)-k b_{-k}, \quad(z \in \partial D) .
\end{aligned}
$$

To simplify the notation, let us assume that $c_{k}:=a_{k}+i b_{k}$ is not only defined for $k \leq 1$ but for all $k \in \mathbf{Z}$ with $c_{k}=0$ for $k \geq 2$ and $k=0$. After some easy algebra, we get that for all $k \in \mathbf{Z}$ and all $\theta \in \mathbf{R} / 2 \pi$ :

$$
\begin{array}{r}
\Re\left(z^{k+1} \phi^{\prime}(\mathbf{c})\left(e^{i \theta}\right)\right)+k a_{-k}=\sum_{j \geq 1}\left[(j-k) a_{j-k}-(j+k) a_{-j-k}\right] \cos (j \theta) \\
+\left[(k-j) b_{j-k}-(j+k) b_{-j-k}\right] \sin (j \theta), \\
\Im\left(z^{k+1} \phi^{\prime}(\mathbf{c})\left(e^{i \theta}\right)\right)+k b_{-k}=\sum_{j \geq 1}\left[(j-k) b_{j-k}-(j+k) b_{-j-k}\right] \cos (j \theta) \\
+\left[(j-k) a_{j-k}+(j+k) a_{-j-k}\right] \sin (j \theta),
\end{array}
$$

and

$$
\begin{aligned}
\Im\left(\overline{\phi(\mathbf{c})\left(e^{i \theta}\right)} e^{i \theta} \phi^{\prime}(\mathbf{c})\left(e^{i \theta}\right)\right)=\sum_{k \geq 1} k\left(\sum_{j \in \mathbf{Z}}\left[a_{j} b_{j+k}-b_{j} a_{j+k}\right]\right) \cos (k \theta) \\
+k\left(\sum_{j \in \mathbf{Z}}\left[a_{k+j} a_{j}+b_{k+j} b_{j}\right]\right) \sin (k \theta) .
\end{aligned}
$$


We deduce the following expressions for the elementary potentials defined in $\Omega:=\mathbf{R}^{2} \backslash \bar{D}$ (in polar coordinates):

$$
\begin{aligned}
& \xi_{1}^{r}(\mathbf{c})(r, \theta)=\sum_{j \geq 1}\left(\mu_{0, j}^{1} \cos (j \theta)+\mu_{0, j}^{2} \sin (j \theta)\right) r^{-j}, \\
& \xi_{2}^{r}(\mathbf{c})(r, \theta)=\sum_{j \geq 1}\left(\nu_{0, j}^{1} \cos (j \theta)+\nu_{0, j}^{2} \sin (j \theta)\right) r^{-j}, \\
& \xi_{3}^{r}(\mathbf{c})(r, \theta)=\sum_{j \geq 1}\left(\alpha_{j}^{1} \cos (j \theta)+\alpha_{j}^{2} \sin (j \theta)\right) r^{-j}, \\
& \xi_{k}^{a}(\mathbf{c})(r, \theta)=\sum_{j \geq 1}\left(\mu_{k, j}^{1} \cos (j \theta)+\mu_{k, j}^{2} \sin (j \theta)\right) r^{-j}, \\
& \xi_{k}^{b}(\mathbf{c})(r, \theta)=\sum_{j \geq 1}\left(\nu_{k, j}^{1} \cos (j \theta)+\nu_{k, j}^{2} \sin (j \theta)\right) r^{-j},
\end{aligned}
$$

where the sequences of real numbers $\left(\alpha_{k}^{1}\right)_{k \geq 1}$ and $\left(\alpha_{k}^{2}\right)_{k \geq 1}$ are defined by:

$$
\begin{array}{rlrl}
\alpha_{k}^{1} & =\sum_{j \in \mathbf{Z}} b_{j+k} a_{j}-a_{j+k} b_{j}, & \alpha_{1}^{2}=-\sum_{j \in \mathbf{Z}} a_{j+1} a_{j}+b_{j+1} b_{j}, \\
\alpha_{k}^{2}=\sum_{j \in \mathbf{Z}} a_{j+k} a_{j}+b_{j+k} b_{j}, & \alpha_{k}^{2}=-\sum_{j \in \mathbf{Z}} a_{j+k} a_{j}+b_{j+k} b_{j},
\end{array}
$$

and the sequences $\left(\mu_{k, j}^{l}\right)_{j \geq 1}$ and $\left(\mu_{k, j}^{l}\right)_{j \geq 1}(l=1,2, k \in \mathbf{Z}, j \geq 1)$ by:

$$
\begin{array}{ll}
\mu_{k, j}^{1}=(k / j+1) a_{k+j}+(k / j-1) a_{k-j}, & \nu_{k, j}^{1}=(k / j+1) b_{k+j}+(k / j-1) b_{k-j}, \\
\mu_{k, j}^{2}=(k / j+1) b_{k+j}-(k / j-1) b_{k-j}, & \nu_{k, j}^{2}=-(k / j+1) a_{k+j}+(k / j-1) a_{k-j} .
\end{array}
$$

From now on, we will denote, for any $k \in \mathbf{Z}, \boldsymbol{\mu}_{k}:=\left(\left(\mu_{k, j}^{1}\right)_{j \geq 1},\left(\mu_{k, j}^{2}\right)_{j \geq 1}\right)$ (a pair of real sequences) and likewise $\boldsymbol{\nu}_{k}:=\left(\left(\nu_{k, j}^{1}\right)_{j \geq 1},\left(\nu_{k, j}^{2}\right)_{j \geq 1}\right)$ and $\boldsymbol{\alpha}:=\left(\left(\alpha_{j}^{1}\right)_{j \geq 1},\left(\alpha_{j}^{2}\right)_{j \geq 1}\right)$.

3.1.2. Kirchhoff's law. In this paragraph, we are concerned with expressing the potential $\xi$ as a linear combination of the elementary potentials. We wish also to prove that they are regular with respect to the shape variable. To this purpose, we introduce $\xi^{r}:=\dot{r}_{1}^{*} \xi_{1}^{r}+\dot{r}_{2}^{*} \xi_{2}^{r}+\omega \xi_{3}^{r}$ and for all $\mathbf{c} \in \mathcal{D}$ and $\dot{\mathbf{c}} \in \mathcal{S}$ :

$$
\left\langle\xi^{d}(\mathbf{c}), \dot{\mathbf{c}}\right\rangle=\sum_{k \leq 1} \dot{a}_{k} \xi_{k}^{a}(\mathbf{c})+\dot{b}_{k} \xi_{k}^{b}(\mathbf{c})
$$

Observe that in this sum, $\xi_{0}^{a}(\mathbf{c})=\xi_{0}^{b}(\mathbf{c})=0$ for all $\mathbf{c} \in \mathcal{D}$. The following lemma generalizes Kirchhoff's law to the infinite dimensional case we are dealing with:

Lemma 3.1. For all $\mathbf{c} \in \mathcal{D}$ and $\dot{\mathbf{c}} \in \mathcal{S}$, the function $\left\langle\xi^{d}(\mathbf{c}), \dot{\mathbf{c}}\right\rangle$ is well defined as element of $H_{N}^{1}(\Omega)$. It is harmonic in $\Omega$ and satisfies the Neumann boundary condition:

$$
\partial_{n}\left\langle\xi^{d}(\mathbf{c}), \dot{\mathbf{c}}\right\rangle=\sum_{k \leq 1} \dot{a}_{k} \partial_{n} \xi_{k}^{a}(\mathbf{c})+\dot{b}_{k} \partial_{n} \xi_{k}^{b}(\mathbf{c}), \quad \text { on } \partial D .
$$

Besides, for all $\mathbf{c} \in \mathcal{D}$, the linear map $\dot{\mathbf{c}} \in \mathcal{S} \mapsto\left\langle\xi^{d}(\mathbf{c}), \dot{\mathbf{c}}\right\rangle \in H_{N}^{1}(\Omega)$ is continuous. For any $\mathbf{c} \in \mathcal{D}$, all the elementary potentials $\xi_{j}^{r}(\mathbf{c})(j=1,2,3)$ and $\xi_{k}^{a}(\mathbf{c}), \xi_{k}^{b}(\mathbf{c})(k \leq 1)$ are well defined as elements 
of $H_{N}^{1}(\Omega)$ so they can be considered as functions of $\mathbf{c} \in \mathcal{D}$ valued in $H_{N}^{1}(\Omega)$. The last assertion of the lemma tells us that for all $\mathbf{c} \in \mathcal{D}, \xi^{d}(\mathbf{c})$ can be seen as a linear continuous mapping from $\mathcal{S}$ into $H_{N}^{1}(\Omega)$. So, $\xi^{d}$ can be seen as a function of $\mathbf{c}$ valued in $\mathcal{L}\left(\mathcal{S}, H_{N}^{1}(\Omega)\right)$. Using the notation of the Appendix B, we claim:

THEOREM 3.2. The following properties hold:

1. Decomposition: For any allowable shape function $t \in[0, T] \mapsto \mathbf{c}(t) \in \mathcal{D}$ :

$$
\xi=\xi^{r}+\left\langle\xi^{d}(\mathbf{c}), \dot{\mathbf{c}}\right\rangle \quad \text { in } H_{N}^{1}(\Omega) .
$$

2. Regularity: $\xi_{j}^{r} \in \mathcal{P}\left(\mathcal{D}, H_{N}^{1}(\Omega)\right)(j=1,2,3), \xi_{k}^{a}, \xi_{k}^{b} \in \mathcal{P}\left(\mathcal{D}, H_{N}^{1}(\Omega)\right)(k \leq 1)$ and $\xi^{d} \in$ $\mathcal{P}\left(\mathcal{D}, \mathcal{L}\left(\mathcal{S}, H_{N}^{1}(\Omega)\right)\right)$.

As already mentioned, we refer to [7] for the proofs of Lemma 3.1 and Theorem 3.2.

3.2. Mass matrices. The notion of mass matrix can be defined as the polarization of the kinetic energy of the system, seen as a quadratic form. Remember that in our case, the kinetic energy coincides with the Lagrangian function defined in Subsection 2.5 and that, for any fixed $\mathbf{c} \in \mathcal{D}, L(\mathbf{c})$ is a quadratic form in $\left(\dot{\mathbf{q}}^{*}, \dot{\mathbf{c}}\right) \in \mathbf{R}^{3} \times \mathcal{S}$. We define then $\mathbb{M}(\mathbf{c})$ as being the bilinear symmetric form on $\left(\mathbf{R}^{3} \times \mathcal{S}\right) \times\left(\mathbf{R}^{3} \times \mathcal{S}\right)$ such that:

$$
L\left(\mathbf{c}, \dot{\mathbf{q}}^{*}, \dot{\mathbf{c}}\right)=\frac{1}{2}\left\langle\mathbb{M}(\mathbf{c}),\left(\dot{\mathbf{q}}^{*}, \dot{\mathbf{c}}\right),\left(\dot{\mathbf{q}}^{*}, \dot{\mathbf{c}}\right)\right\rangle .
$$

Then we decompose it into $\mathbb{M}^{r}(\mathbf{c})$, a bilinear symmetric form on $\mathbf{R}^{3} \times \mathbf{R}^{3}$ (that can be identified with an actual $3 \times 3$ symmetric matrix $), \mathbb{N}(\mathbf{c})$ a bilinear form on $\mathcal{S} \times \mathbf{R}^{3}$ and $\mathbb{M}^{d}(\mathbf{c})$ a bilinear symmetric form on $\mathcal{S} \times \mathcal{S}$ such that:

$$
\left\langle\mathbb{M}(\mathbf{c}),\left(\dot{\mathbf{c}}, \dot{\mathbf{q}}^{*}\right),\left(\dot{\mathbf{c}}, \dot{\mathbf{q}}^{*}\right)\right\rangle=\left\langle\mathbb{M}^{r}(\mathbf{c}), \dot{\mathbf{q}}^{*}, \dot{\mathbf{q}}^{*}\right\rangle+\left\langle\mathbb{M}^{d}(\mathbf{c}), \dot{\mathbf{c}}, \dot{\mathbf{c}}\right\rangle+2\left\langle\mathbb{N}(\mathbf{c}), \dot{\mathbf{c}}, \dot{\mathbf{q}}^{*}\right\rangle .
$$

We are interested in determining explicit expressions for $\mathbb{M}^{r}(\mathbf{c}), \mathbb{N}(\mathbf{c})$ and $\mathbb{M}^{d}(\mathbf{c})$ and in studying their dependence with respect to the control variable $\mathbf{c}$.

3.2.1. Mass matrix related to the rigid motion. First, we consider $\mathbb{M}^{r}(\mathbf{c})$, the classical mass matrix of the body associated to its rigid motion. The decomposition of the potential function obtained in $\S 3.1 .2$ leads us to introduce the symmetric $3 \times 3$ matrix:

$$
\mathbb{M}^{r}(\mathbf{c}):=\left[\begin{array}{ccc}
m & 0 & 0 \\
0 & m & 0 \\
0 & 0 & I(\mathbf{c})
\end{array}\right]+\left[\begin{array}{ccc}
\int_{\Omega} \nabla \xi_{1}^{r}(\mathbf{c}) \cdot \nabla \xi_{1}^{r}(\mathbf{c}) \mathrm{d} m_{f}^{0} & \cdots \int_{\Omega} \nabla \xi_{1}^{r}(\mathbf{c}) \cdot \nabla \xi_{3}^{r}(\mathbf{c}) \mathrm{d} m_{f}^{0} \\
\vdots & \vdots \\
\int_{\Omega} \nabla \xi_{3}^{r}(\mathbf{c}) \cdot \nabla \xi_{1}^{r}(\mathbf{c}) \mathrm{d} m_{f}^{0} & \cdots \int_{\Omega} \nabla \xi_{3}^{r}(\mathbf{c}) \cdot \nabla \xi_{3}^{r}(\mathbf{c}) \mathrm{d} m_{f}^{0}
\end{array}\right]
$$

where we recall that $m>0$ is the constant mass of the body and $I(\mathbf{c})$ is its inertia momentum given in (2.11). The kinetic energy resulting from the rigid displacement of the body can be written as the matrix-vectors product: $(1 / 2)\left(\dot{\mathbf{r}}^{*}, \omega\right) \mathbb{M}^{r}(\mathbf{c})\left(\dot{\mathbf{r}}^{*}, \omega\right)^{T}$. The latter matrix in the right hand side of (3.9) is usually referred to as an added mass matrix, relating here to the rigid motion of the body.

We are dealing now with the kinetic energy in connexion with the shape changes, considering separately the infinite and finite dimensional cases.

3.2.2. Infinite dimensional case. To give the expressions of the elements of the matrices $\mathbb{N}(\mathbf{c})$ and $\mathbb{M}^{d}(\mathbf{c})$, we define $\mathbf{a}^{j}=\left(a_{k}^{j}\right)_{k \leq 1}$ and $\mathbf{b}^{j}=\left(b_{k}^{j}\right)_{k \leq 1} \in \ell^{2}(\mathbf{C})(j \leq 1)$, the complex sequences 
such that $a_{k}^{j}=\delta_{k}^{j}$ (the Kronecker symbol) and $b_{k}^{j}=i \delta_{k}^{j}$. Using the canonical basis $\left\{\mathbf{f}^{1}, \mathbf{f}^{2}, \mathbf{f}^{3}\right\}$ of $\mathbf{R}^{3}$ and the Schauder basis $\left\{\mathbf{a}^{j}, \mathbf{b}^{j}, j \leq 1\right\}$ of $\ell^{2}(\mathbf{C})$, we can now define, for any $1 \leq k \leq 3$ and $j \leq 1$ :

$$
\begin{aligned}
\left\langle\mathbb{N}(\mathbf{c}), \mathbf{a}^{j}, \mathbf{f}^{k}\right\rangle & :=\int_{\Omega} \nabla \xi_{k}^{r}(\mathbf{c}) \cdot \nabla \xi_{j}^{a}(\mathbf{c}) \mathrm{d} m_{f}^{0}, \\
\left\langle\mathbb{N}(\mathbf{c}), \mathbf{b}^{j}, \mathbf{f}^{k}\right\rangle & :=\int_{\Omega} \nabla \xi_{k}^{r}(\mathbf{c}) \cdot \nabla \xi_{j}^{b}(\mathbf{c}) \mathrm{d} m_{f}^{0} .
\end{aligned}
$$

The entries of $\mathbb{M}^{d}(\mathbf{c})$ are, for all $j, k \leq 1$ and $j, k \neq 0$ :

$$
\begin{aligned}
\left\langle\mathbb{M}^{d}(\mathbf{c}), \mathbf{a}^{j}, \mathbf{a}^{k}\right\rangle & :=\int_{\Omega} \nabla \xi_{j}^{a}(\mathbf{c}) \cdot \nabla \xi_{k}^{a}(\mathbf{c}) \mathrm{d} m_{f}^{0}+\frac{\pi \varrho_{0} \delta_{k}^{j}}{|k|+1}, \\
\left\langle\mathbb{M}^{d}(\mathbf{c}), \mathbf{b}^{j}, \mathbf{b}^{k}\right\rangle & :=\int_{\Omega} \nabla \xi_{j}^{b}(\mathbf{c}) \cdot \nabla \xi_{k}^{b}(\mathbf{c}) \mathrm{d} m_{f}^{0}+\frac{\pi \varrho_{0} \delta_{k}^{j}}{|k|+1}, \\
\left\langle\mathbb{M}^{d}(\mathbf{c}), \mathbf{a}^{j}, \mathbf{b}^{k}\right\rangle & :=\int_{\Omega} \nabla \xi_{j}^{a}(\mathbf{c}) \cdot \nabla \xi_{k}^{b}(\mathbf{c}) \mathrm{d} m_{f}^{0} .
\end{aligned}
$$

The elements corresponding to $j=0$ or $k=0$ are all equal to 0 .

3.2.3. Finite dimensional case. We assume now that $\mathbf{c}$ and $\dot{\mathbf{c}}$ have only a finite number of (possibly) non-zero elements, say the $N+1$ firsts $(N \geq 0)$. As explained in the Appendix A, in this case $\mathbf{c}, \dot{\mathbf{c}} \in \mathcal{S}_{N}$ and we identify this space with $\mathbf{R}^{2(N+1)}$. The bilinear mappings $\mathbb{N}(\mathbf{c})$ and $\mathbb{M}^{d}(\mathbf{c})$ can then be identified with actual matrices of sizes $3 \times 2(N+1)$ and $2(N+1) \times 2(N+1)$ respectively. Thus, we have:

$$
\mathbb{N}(\mathbf{c}):=\left[\begin{array}{l}
\int_{\Omega} \nabla \xi_{1}^{r}(\mathbf{c}) \cdot \nabla \xi_{1}^{a}(\mathbf{c}) \mathrm{d} m_{f}^{0} \cdots \int_{\Omega} \nabla \xi_{1}^{r}(\mathbf{c}) \cdot \nabla \xi_{-N}^{b}(\mathbf{c}) \mathrm{d} m_{f}^{0} \\
\int_{\Omega} \nabla \xi_{2}^{r}(\mathbf{c}) \cdot \nabla \xi_{1}^{a}(\mathbf{c}) \mathrm{d} m_{f}^{0} \cdots \int_{\Omega} \nabla \xi_{2}^{r}(\mathbf{c}) \cdot \nabla \xi_{-N}^{b}(\mathbf{c}) \mathrm{d} m_{f}^{0} \\
\int_{\Omega} \nabla \xi_{3}^{r}(\mathbf{c}) \cdot \nabla \xi_{1}^{a}(\mathbf{c}) \mathrm{d} m_{f}^{0} \cdots \int_{\Omega} \nabla \xi_{3}^{r}(\mathbf{c}) \cdot \nabla \xi_{-N}^{b}(\mathbf{c}) \mathrm{d} m_{f}^{0}
\end{array}\right],
$$

while $\mathbb{M}^{d}(\mathbf{c})$ reads:

$$
\begin{aligned}
& \mathbb{M}^{d}(\mathbf{c}):=\left[\begin{array}{cccc}
\int_{\Omega} \nabla \xi_{1}^{a}(\mathbf{c}) \cdot \nabla \xi_{1}^{a}(\mathbf{c}) \mathrm{d} m_{f}^{0} & \cdots & \int_{\Omega} \nabla \xi_{1}^{a}(\mathbf{c}) \cdot \nabla \xi_{-N}^{b}(\mathbf{c}) \mathrm{d} m_{f}^{0} \\
\int_{\Omega} \nabla \xi_{1}^{b}(\mathbf{c}) \cdot \nabla \xi_{1}^{a}(\mathbf{c}) \mathrm{d} m_{f}^{0} & \cdots & \int_{\Omega} \nabla \xi_{1}^{b}(\mathbf{c}) \cdot \nabla \xi_{-N}^{b}(\mathbf{c}) \mathrm{d} m_{f}^{0} \\
\vdots & & & \vdots \\
\int_{\Omega} \nabla \xi_{-N}^{a}(\mathbf{c}) \cdot \nabla \xi_{1}^{a}(\mathbf{c}) \mathrm{d} m_{f}^{0} & \cdots & \int_{\Omega} \nabla \xi_{-N}^{a}(\mathbf{c}) \cdot \nabla \xi_{-N}^{b}(\mathbf{c}) \mathrm{d} m_{f}^{0} \\
\int_{\Omega} \nabla \xi_{-N}^{b}(\mathbf{c}) \cdot \nabla \xi_{1}^{a}(\mathbf{c}) \mathrm{d} m_{f}^{0} & \cdots & \int_{\Omega} \nabla \xi_{-N}^{b}(\mathbf{c}) \cdot \nabla \xi_{-N}^{b}(\mathbf{c}) \mathrm{d} m_{f}^{0}
\end{array}\right]+ \\
& \pi \varrho_{0} \operatorname{diag}(1 / 2,1 / 2,1 / 2,1 / 2,1 / 3,1 / 3,1 / 4,1 / 4, \ldots, 1 / N, 1 / N) .
\end{aligned}
$$

In these definitions, the index $j$ of $\nabla \xi_{j}^{a}$ and $\nabla \xi_{j}^{b}$ in the integrals ranges from 1 to $-N$ and there is no term corresponding to the index $j=0$. Remark that in the finite dimensional case, the overall mass matrix $\mathbb{M}(\mathbf{c})$ can also be identified with an actual $(3+2(N+1)) \times(3+2(N+1))$ bloc matrix defined by:

$$
\mathbb{M}(\mathbf{c}):=\left[\begin{array}{cc}
\mathbb{M}^{r}(\mathbf{c}) & \mathbb{N}(\mathbf{c}) \\
\mathbb{N}(\mathbf{c})^{T} & \mathbb{M}^{d}(\mathbf{c})
\end{array}\right]
$$


3.2.4. Regularity of the Mass Matrices and Lagrangian function. As a straightforward consequence of Theorem 3.2 we get, with the notation of Appendix B (see [7] for details):

Theorem 3.3. $\mathbb{M}^{r} \in \mathcal{P}\left(\mathcal{D}, \mathcal{L}_{2}\left(\mathbf{R}^{3} \times \mathbf{R}^{3}\right)\right), \mathbb{N} \in \mathcal{P}\left(\mathcal{D}, \mathcal{L}_{2}\left(\mathbf{R}^{3} \times \mathcal{S}\right)\right)$ and $\mathbb{M}^{d} \in \mathcal{P}\left(\mathcal{D}, \mathcal{L}_{2}(\mathcal{S} \times \mathcal{S})\right)$. It entails that $\mathbb{M} \in \mathcal{P}\left(\mathcal{D}, \mathcal{L}_{2}\left(\left(\mathbf{R}^{3} \times \mathcal{S}\right) \times\left(\mathbf{R}^{3} \times \mathcal{S}\right)\right)\right)$ and the Lagrangian function is smooth in all of its variables. Furthermore, the mapping $\mathbf{c} \in \mathcal{D} \mapsto \mathbb{M}^{r}(\mathbf{c})^{-1} \in \mathcal{L}\left(\mathbf{R}^{3}, \mathbf{R}^{3}\right)$ is also well-defined and analytic.

3.2.5. Explicit computation of the mass matrices. The entries of the mass matrices defined in Subsection 3.2 can be now easily deduced from the expressions (3.6) of the elementary potentials. Indeed, let us consider for instance the first element of the matrix $\mathbb{M}^{r}(\mathbf{c})$. Applying Green's formula, we get:

$$
\int_{\Omega} \nabla \xi_{1}^{r}(\mathbf{c}) \cdot \nabla \xi_{1}^{r}(\mathbf{c}) \mathrm{d} m_{f}=-\varrho_{f} \int_{\partial D} \xi_{1}^{r}(\mathbf{c}) \frac{\partial \xi_{1}^{r}}{\partial r}(\mathbf{c}) \mathrm{d} \sigma
$$

and then:

$$
\int_{\Omega} \nabla \xi_{1}^{r}(\mathbf{c}) \cdot \nabla \xi_{1}^{r}(\mathbf{c}) \mathrm{d} m_{f}=\pi \varrho_{f} \sum_{j \geq 1} j\left|\mu_{0, j}\right|^{2}
$$

For any two pair of sequences $\boldsymbol{v}:=\left(\left(v_{j}^{1}\right)_{j \geq 1},\left(v_{j}^{2}\right)_{j \geq 1}\right)$ and $\varsigma:=\left(\left(\varsigma_{j}^{1}\right)_{j \geq 1},\left(\varsigma_{j}^{2}\right)_{j \geq 1}\right)$ of real numbers, we introduce the notation:

$$
\boldsymbol{v} \cdot \boldsymbol{\varsigma}:=\sum_{k \geq 1} k\left(v_{k}^{1} \varsigma_{k}^{1}+v_{k}^{2} \varsigma_{k}^{2}\right) \quad \text { and } \quad|\boldsymbol{v}|^{2}:=\boldsymbol{v} \cdot \boldsymbol{v} .
$$

Taking into account the expressions (3.9), (2.11) and (3.6), we can give the expression of the mass matrices in a convenient short form:

$$
\mathbb{M}^{r}(\mathbf{c})=\varrho_{0} \pi\left[\begin{array}{ccc}
1 & 0 & 0 \\
0 & 1 & 0 \\
0 & 0 & \sum_{k \leq 1} \frac{\left|c_{k}\right|^{2}}{|k|+1}
\end{array}\right]+\varrho_{f} \pi\left[\begin{array}{ccc}
\left|\boldsymbol{\mu}_{0}\right|^{2} & \boldsymbol{\mu}_{0} \cdot \boldsymbol{\nu}_{0} & \boldsymbol{\mu}_{0} \cdot \boldsymbol{\alpha} \\
\boldsymbol{\mu}_{0} \cdot \boldsymbol{\nu}_{0} & \left|\boldsymbol{\nu}_{0}\right|^{2} & \boldsymbol{\nu}_{0} \cdot \boldsymbol{\alpha} \\
\boldsymbol{\mu}_{0} \cdot \boldsymbol{\alpha} & \boldsymbol{\nu}_{0} \cdot \boldsymbol{\alpha} & |\boldsymbol{\alpha}|^{2}
\end{array}\right]
$$

and likewise the elements (3.10) of the mass matrix $\mathbb{N}$ read, for all $k \leq 1, k \neq 0$ :

$$
\begin{gathered}
\left\langle\mathbb{N}(\mathbf{c}), \mathbf{a}^{k}, \mathbf{f}^{j}\right\rangle= \begin{cases}\varrho_{f} \pi \boldsymbol{\mu}_{0} \cdot \boldsymbol{\mu}_{k} & \text { if } j=1 \\
\varrho_{f} \pi \boldsymbol{\nu}_{0} \cdot \boldsymbol{\mu}_{k} & \text { if } j=2 \\
\varrho_{f} \pi \boldsymbol{\alpha} \cdot \boldsymbol{\mu}_{k} & \text { if } j=3,\end{cases} \\
\left\langle\mathbb{N}(\mathbf{c}), \mathbf{b}^{k}, \mathbf{f}^{j}\right\rangle= \begin{cases}\varrho_{f} \pi \boldsymbol{\mu}_{0} \cdot \boldsymbol{\nu}_{k} & \text { if } j=1 \\
\varrho_{f} \pi \boldsymbol{\nu}_{0} \cdot \boldsymbol{\nu}_{k} & \text { if } j=2 \\
\varrho_{f} \pi \boldsymbol{\alpha} \cdot \boldsymbol{\nu}_{k} & \text { if } j=3 .\end{cases}
\end{gathered}
$$

At last, the expressions of the elements (3.11) of $\mathbb{M}^{d}(\mathbf{c})$ read, for all $j, k \leq 1$ with $j, k \neq 0$ :

$$
\begin{aligned}
\left\langle\mathbb{M}^{d}(\mathbf{c}), \mathbf{a}^{j}, \mathbf{a}^{k}\right\rangle & =\varrho_{f} \pi \boldsymbol{\mu}_{j} \cdot \boldsymbol{\mu}_{k}+\frac{\pi \varrho_{0} \delta_{k}^{j}}{k+1}, \quad\left\langle\mathbb{M}^{d}(\mathbf{c}), \mathbf{a}^{j}, \mathbf{b}^{k}\right\rangle(\mathbf{c})=\varrho_{f} \pi \boldsymbol{\mu}_{j} \cdot \boldsymbol{\nu}_{k}, \\
\left\langle\mathbb{M}^{d}(\mathbf{c}), \mathbf{b}^{j}, \mathbf{b}^{k}\right\rangle & =\varrho_{f} \pi \boldsymbol{\nu}_{j} \cdot \boldsymbol{\nu}_{k}+\frac{\pi \varrho_{0} \delta_{k}^{j}}{k+1} .
\end{aligned}
$$


3.3. Equation of motion. Following the method explained in [17, chap VI, pages 160-201], we introduce $\mathbf{P}$ and $\Pi$ the translational and angular impulses as well as $\mathbf{L}$ and $\Lambda$, the impulses relating to the deformations:

$$
\left[\begin{array}{l}
\mathbf{P} \\
\Pi
\end{array}\right]:=\mathbb{M}^{r}(\mathbf{c})\left[\begin{array}{c}
\dot{\mathbf{r}}^{*} \\
\omega
\end{array}\right] \quad \text { and } \quad\left[\begin{array}{l}
\mathbf{L} \\
\Lambda
\end{array}\right]:=\langle\mathbb{N}(\mathbf{c}), \dot{\mathbf{c}}\rangle
$$

In these identities, both left hand side terms can be identified with elements of $\mathbf{R}^{3}$. We compute that for all $\dot{\mathbf{p}}:=(\dot{\mathbf{c}}, \tilde{\omega})^{T} \in \mathbf{R}^{3}$ :

$$
\frac{d}{d t} \frac{\partial}{\partial \dot{\mathbf{q}}}\left[\begin{array}{c}
\dot{\mathbf{r}}^{*} \\
\omega
\end{array}\right] \cdot \dot{\mathbf{p}}-\frac{\partial}{\partial \mathbf{q}}\left[\begin{array}{c}
\dot{\mathbf{r}}^{*} \\
\omega
\end{array}\right] \cdot \dot{\mathbf{p}}=\left[\begin{array}{c}
\tilde{\omega}\left(\dot{\mathbf{r}}^{*}\right)^{\perp}-\omega\left(\dot{\mathbf{c}}^{*}\right)^{\perp} \\
0
\end{array}\right] .
$$

We next easily obtain that:

$$
\frac{d}{d t} \frac{\partial L}{\partial \dot{\mathbf{q}}} \cdot \dot{\mathbf{p}}-\frac{\partial L}{\partial \mathbf{q}} \cdot \dot{\mathbf{p}}=\frac{d}{d t}\left[\begin{array}{l}
\mathbf{P}+\mathbf{L} \\
\Pi+\Lambda
\end{array}\right] \cdot\left[\begin{array}{c}
\dot{\mathbf{c}}^{*} \\
\tilde{\omega}
\end{array}\right]+\left[\begin{array}{l}
\mathbf{P}+\mathbf{L} \\
\Pi+\Lambda
\end{array}\right] \cdot\left[\begin{array}{c}
\tilde{\omega}\left(\dot{\mathbf{r}}^{*}\right)^{\perp}-\omega\left(\dot{\mathbf{c}}^{*}\right)^{\perp} \\
0
\end{array}\right] .
$$

According to Theorem 3.3, the Lagrangian function is smooth with respect to all of its variables, allowing all of the derivatives to be computed. Invoking the least action principle, the EulerLagrange equation of motion is (see e.g. [19, Theorem 7.3.3 page 187]):

$$
\frac{d}{d t} \frac{\partial L}{\partial \dot{\mathbf{q}}} \cdot \dot{\mathbf{p}}-\frac{\partial L}{\partial \mathbf{q}} \cdot \dot{\mathbf{p}}=0, \quad \forall \dot{\mathbf{p}} \in \mathbf{R}^{3}
$$

In our case we get:

$$
\begin{aligned}
\frac{d}{d t}(\mathbf{P}+\mathbf{L})+\omega(\mathbf{P}+\mathbf{L})^{\perp} & =0, \\
\frac{d}{d t}(\Pi+\Lambda)-\dot{\mathbf{r}}^{*} \cdot(\mathbf{P}+\mathbf{L})^{\perp} & =0 .
\end{aligned}
$$

If at the time $t=0$, the impulse of the system is null:

$$
\left[\begin{array}{l}
\mathbf{P}+\mathbf{L} \\
\Pi+\Lambda
\end{array}\right]=0
$$

this relation remains true for all $t>0$ since the Cauchy-Lipschitz Theorem ensures the uniqueness of the solution of system (3.19). We obtain here the equation:

$$
\dot{\mathbf{q}}^{*}=-\left(\mathbb{M}^{r}(\mathbf{c})\right)^{-1}\langle\mathbb{N}(\mathbf{c}), \dot{\mathbf{c}}\rangle
$$

We introduce the $3 \times 3$ block matrix:

$$
\mathcal{R}(\theta):=\left[\begin{array}{cc}
R(\theta) & 0 \\
0 & 1
\end{array}\right]
$$

and since $\dot{\mathbf{q}}^{*}=\mathcal{R}(\theta)^{T} \dot{\mathbf{q}}$, we can rewrite (3.20) in the form:

$$
\dot{\mathbf{q}}=-\mathcal{R}(\theta)\left(\mathbb{M}^{r}(\mathbf{c})\right)^{-1}\langle\mathbb{N}(\mathbf{c}), \dot{\mathbf{c}}\rangle, \quad(t>0),
$$

which allows the rigid displacement to be computed, knowing the shape changes. From Theorem 3.3, we deduce:

Proposition 3.4 (Well-posedness). For any $\mu>0$, for any physically allowable control function $\mathbf{c}:[0, T] \rightarrow \mathcal{A}^{\bullet}(\mu)$ and for any initial condition $\left(\mathbf{r}_{0}, \theta_{0}\right) \in \mathbf{R}^{2} \times \mathbf{R} / 2 \pi$, there exists one unique smooth solution to Equation (3.21) defined on $[0, T]$. 
4. Equation of Elasticity. We assume that the swimmer is hyperelastic. A hyperelastic (or Green elastic material) is an ideally elastic material for which the stress-strain relationship follows from a strain energy density function. It is, for instance, the model of material most suited to the analysis of elastomers.

In the following, we assume that there exists a scalar valued volumetric strain energy function $U(z, \mathbb{F}(\mathbf{c})(z))$ (recall that $\mathbb{F}(\mathbf{c})(z)$ is the deformation tensor) defined in the reference configuration $D$ and encapsulating all the information regarding the material behavior. From $U$, we can define the potential energy of elasticity:

$$
W(\mathbf{c}):=\int_{\Omega} U(z, \mathbb{F}(\mathbf{c})(z)) \mathrm{d} z, \quad(\mathbf{c} \in \mathcal{D}),
$$

which is actually the only quantity we will need to derive the equations governing the elastic motion of the body. Classical constitutive models for $2 \mathrm{D}$ isotropic elastic materials are usually expressed in terms of the invariants $I_{1}(\mathbf{c})$ and $I_{2}(\mathbf{c})$ of $\mathbb{B}(\mathbf{c}):=\mathbb{F}(\mathbf{c}) \mathbb{F}(\mathbf{c})^{T}$, the left Cauchy-Green tensor. They are defined by $I_{1}(\mathbf{c}):=\operatorname{Tr}(\mathbb{B}(\mathbf{c}))$ and $I_{2}(\mathbf{c}):=\operatorname{det}(\mathbb{B}(\mathbf{c}))=J(\mathbf{c})^{2}$. The modified invariants $\bar{I}_{1}(\mathbf{c}):=I_{1}(\mathbf{c}) / J(\mathbf{c})$ and $\bar{I}_{2}(\mathbf{c}):=J(\mathbf{c})$ can be used as well. Let us mention for instance the Neo-Hookean hyperelastic material (from the book [18]), whose the strain energy function is $U(z, \mathbf{c})=a I_{1}(\mathbf{c})(z)$ for some $a>0$. The relating potential energy of elasticity would be:

$$
W(\mathbf{c})=\pi a\left(\sum_{k \leq 1} \frac{\left|c_{k}\right|^{2}}{|k|+1}\right) .
$$

In the following, we will take the most of the existence of the shape variable and deal with quite general forms for the potential energy $W$.

\subsection{Infinite dimensional case.}

4.1.1. Potential energy of elasticity. Recall that to be allowable, the shape variable $\mathbf{c}$ has to remain within $\mathcal{D}$. Let us give a physical interpretation of this condition: According to the expression (2.4), we deduce that $J(\mathbf{c})(z)>0$ for all $z \in \bar{D}$ as long as $\mathbf{c}$ remains in $\mathcal{D}$. The maximum principle tells us that

$$
\min _{z \in \bar{D}} J(\mathbf{c})(z)=\left|c_{1}\right|^{2}-\max _{z \in \partial D}\left|\sum_{k \geq 0}(k+1) c_{-k-1} \bar{z}^{k}\right|^{2}=\left|c_{1}\right|^{2}-\max _{z \in \partial D}\left|\sum_{k \geq 1} k c_{-k} z^{k}\right|^{2} .
$$

So, when $\mathbf{c} \rightarrow \partial \mathcal{D}, J(\mathbf{c})(z) \rightarrow 0$ at some point $z \in \partial D$ and according to (2.3), it entails that $\varrho^{*}\left(x^{*}\right) \rightarrow+\infty\left(x^{*}=\chi(\mathbf{c})(z) \in \partial \mathcal{B}^{*}\right)$ (in other words, the density of the body becomes infinite). In order to prevent this phenomenon, we wish the potential energy $W$ to satisfy $W(\mathbf{c}) \rightarrow+\infty$ as $\mathbf{c} \rightarrow \partial \mathcal{D}$. On the other hand, we also want the body to tend to recover a given position $\mathbf{c}_{\text {ref }} \in \mathcal{D}$, so we decompose $W$ into two parts: $W=W_{1}+W_{2}$ where

1. $W_{1}(\mathbf{c}):=V\left(\mathbf{c}-\mathbf{c}_{\mathrm{ref}}\right)$ and $V: \mathcal{S} \rightarrow \mathbf{R}$ is a given smooth convex function, the minimum of which is achieved for $\mathbf{c}=0$ (a simple example of such a function could be $V(\mathbf{c})=\|\mathbf{c}\|_{\ell^{2}(\mathbf{C})}^{2}$ );

2. $W_{2}(\mathbf{c}):=(\varepsilon / 2 \alpha) \int_{\partial D} J(\mathbf{c})(z)^{-\alpha} \mathrm{d} \sigma$, where $\varepsilon$ and $\alpha$ are two positive constants (we will see later on how to choose them for $W_{2}$ to meet with the requirement that $W_{2} \rightarrow+\infty$ as $\left.\mathbf{c} \rightarrow \partial \mathcal{D}\right)$. Notice that the equilibrium shape of the body corresponds to the value of $\mathbf{c} \in \mathcal{D}$ minimizing $W$ and can be different from $\mathbf{c}_{\text {ref }}$. Concerning $W_{2}$, one can easily show the following result:

Lemma 4.1. For all $\varepsilon>0$ and $\alpha>0$, the mapping $\mathbf{c} \in \mathcal{D} \mapsto W_{2}(\mathbf{c}) \in \mathbf{R}$ is analytic. 
We can even compute the first derivative of $W_{2}$ with respect to $\mathbf{c}:=\left(c_{k}\right)_{k \leq 1} \in \mathcal{D}\left(c_{k}:=a_{k}+i b_{k}\right)$. Thus, for all $k \leq 1$, we get:

$$
\begin{aligned}
& \frac{\partial W_{2}}{\partial a_{k}}(\mathbf{c})=-\varepsilon k \int_{\partial D} \Re\left(z^{k-1} \sum_{j \geq 0}(j+1) c_{-j-1} \bar{z}^{j}\right) J(\mathbf{c})(z)^{-1-\alpha} \mathrm{d} \sigma, \\
& \frac{\partial W_{2}}{\partial b_{k}}(\mathbf{c})=-\varepsilon k \int_{\partial D} \Im\left(z^{k-1} \sum_{j \geq 0}(j+1) c_{-j-1} \bar{z}^{j}\right) J(\mathbf{c})(z)^{-1-\alpha} \mathrm{d} \sigma .
\end{aligned}
$$

Then we have, for all $\mathbf{c} \in \mathcal{D}$, the following expression for the derivative in the direction of $\tilde{\mathbf{c}} \in \mathcal{S}$ :

$$
\left\langle\frac{\partial W_{2}}{\partial \mathbf{c}}(\mathbf{c}), \tilde{\mathbf{c}}\right\rangle=\sum_{k \leq 1} \frac{\partial W_{2}}{\partial a_{k}}(\mathbf{c}) \tilde{a}_{k}+\frac{\partial W_{2}}{\partial b_{k}}(\mathbf{c}) \tilde{b}_{k} .
$$

4.1.2. Modified Lagrangian. With the notation of Subsection 3.2, the Lagrangian function can now be modified as follows:

$$
L\left(\mathbf{c}, \dot{\mathbf{c}}, \dot{\mathbf{q}}^{*}\right)=\frac{1}{2}\left\langle\mathbb{M}^{r}(\mathbf{c}), \dot{\mathbf{q}}^{*}, \dot{\mathbf{q}}^{*}\right\rangle+\frac{1}{2}\left\langle\mathbb{M}^{d}(\mathbf{c}), \dot{\mathbf{c}}, \dot{\mathbf{c}}\right\rangle+\left\langle\mathbb{N}(\mathbf{c}), \dot{\mathbf{c}}, \dot{\mathbf{q}}^{*}\right\rangle-W(\mathbf{c}) .
$$

Notice that $W$ does not depend on $\mathbf{q}$ and $\dot{\mathbf{q}}$ and hence the equation $\dot{\mathbf{q}}^{*}=-\left(\mathbb{M}^{r}(\mathbf{c})\right)^{-1}\langle\mathbb{N}(\mathbf{c}), \dot{\mathbf{c}}\rangle$ driving the rigid motion still holds. Substituting this expression into (4.3), we obtain a new Lagrangian function depending only on the shape variable:

$$
\mathcal{L}(\mathbf{c}, \dot{\mathbf{c}}):=L\left(\mathbf{c}, \dot{\mathbf{c}},-\left(\mathbb{M}^{r}(\mathbf{c})\right)^{-1}\langle\mathbb{N}(\mathbf{c}), \dot{\mathbf{c}}\rangle\right),
$$

or in short form:

$$
\mathcal{L}(\mathbf{c}, \dot{\mathbf{c}})=\frac{1}{2}\langle\mathbb{K}(\mathbf{c}), \dot{\mathbf{c}}, \dot{\mathbf{c}}\rangle-W(\mathbf{c}),
$$

where the mass matrix $\mathbb{K}(\mathbf{c}) \in \mathcal{L}_{2}(\mathcal{S} \times \mathcal{S})$ is defined for all $\mathbf{c} \in \mathcal{D}$ by:

$$
\left\langle\mathbb{K}(\mathbf{c}), \mathbf{c}_{1}, \mathbf{c}_{2}\right\rangle:=\left\langle\mathbb{M}^{d}(\mathbf{c}), \mathbf{c}_{1}, \mathbf{c}_{2}\right\rangle-\left\langle\mathbb{N}(\mathbf{c}), \mathbf{c}_{1}\right\rangle^{T}\left(\mathbb{M}^{r}(\mathbf{c})\right)^{-1}\left\langle\mathbb{N}(\mathbf{c}), \mathbf{c}_{2}\right\rangle, \quad\left(\mathbf{c}_{1}, \mathbf{c}_{2} \in \mathcal{S}\right) .
$$

According to Theorem 3.3, the mapping $\mathbf{c} \in \mathcal{D} \mapsto \mathbb{K}(\mathbf{c}) \in \mathcal{L}_{2}(\mathcal{S} \times \mathcal{S})$, like the all of the other mass matrices in this article, is analytic.

4.1.3. Constraints. We denote $(\cdot, \cdot)_{\ell^{2}(\mathbf{C})}$ the scalar product in $\ell^{2}(\mathbf{C})$ defined for all $\mathbf{c}^{1}:=$ $\left(c_{k}^{1}\right)_{k \leq 1}$ and $\mathbf{c}^{2}:=\left(c_{k}^{2}\right)_{k \leq 1} \in \mathcal{S}$ by $\left(\mathbf{c}^{1}, \mathbf{c}^{2}\right)_{\ell^{2}(\mathbf{C})}:=\sum_{k \leq 1}\left(a_{k}^{1} a_{k}^{2}+b_{k}^{1} b_{k}^{2}\right)$. According to the Riesz Representation theorem, we can identify $\ell^{2}(\mathbf{C})$ with its dual space and since the injection $\mathcal{S} \subset \ell^{2}(\mathbf{C})$ is continuous and dense, we can use the scalar product of $\ell^{2}(\mathbf{C})$ to express the duality product of $\mathcal{S} \times \mathcal{S}^{\prime}$. Via this identification, we have:

$$
\mathcal{S}^{\prime}:=\left\{\mathbf{c}:=\left(c_{k}\right)_{k \leq 1}: \max _{k \leq 1}\left\{\left|k a_{k}\right|,\left|k b_{k}\right|\right\}<+\infty\right\},
$$

and the classical inclusion chain: $\mathcal{S} \subset \ell^{2}(\mathbf{C}) \equiv \ell^{2}(\mathbf{C})^{\prime} \subset \mathcal{S}^{\prime}$. For all $\mathbf{c}:=\left(c_{k}\right)_{k \leq 1} \in \mathcal{S}$, we denote $G_{1}(\mathbf{c}):=\left(\check{c}_{k}\right)_{k \leq 1}$ and $G_{2}(\mathbf{c}):=\left(\hat{c}_{k}\right)_{k \leq 1}$ the elements of $\ell^{2}(\mathbf{C}) \subset \mathcal{S}^{\prime}$ such that:

$$
\check{c}_{k}:=k c_{k} \quad \text { and } \quad \hat{c}_{k}:=-i \frac{c_{k}}{|k|+1}, \quad(k \leq 1),
$$


and we can rewrite the constraints (2.8) and (2.14) required for a shape variable to be allowable respectively as

$$
\left(G_{1}(\mathbf{c}), \dot{\mathbf{c}}\right)_{\ell^{2}(\mathbf{C})}=0 \quad \text { and } \quad\left(G_{2}(\mathbf{c}), \dot{\mathbf{c}}\right)_{\ell^{2}(\mathbf{C})}=0 .
$$

More generally, we can introduce any additional constraint on the shape variable that can be put into the form $\left(G_{j}(\mathbf{c}), \dot{\mathbf{c}}\right)_{\ell^{2}(\mathbf{C})}=0$ where $G_{j}(j \geq 3)$ is a smooth function from $\mathcal{S}$ into $\ell^{2}(\mathbf{C})$. Let us assume that there is a number $N^{\prime}$ of such constraints $\left(N^{\prime}\right.$ an integer greater or equal to 2$)$. We assume in addition that all of the constraints satisfy:

1. $G_{j}(\mathbf{c}) \neq 0$ for all $\mathbf{c} \in \mathcal{S}$ (i.e. the constraint is always non-degenerated).

2. For all $\mathbf{c} \in \mathcal{S}$, the system $\left\{G_{j}(\mathbf{c}), 1 \leq j \leq N^{\prime}\right\}$ is free in $\ell^{2}(\mathbf{C})$ (the constraints are independent).

Observe that $\left(G_{1}(\mathbf{c}), G_{2}(\mathbf{c})\right)_{\ell^{2}(\mathbf{C})}=0$ for all $\mathbf{c} \in \mathcal{S}$. Actually, under hypotheses (1-2), we can assume without loss of generality that the system $\left\{G_{j}(\mathbf{c}), 1 \leq j \leq N^{\prime}\right\}$ is orthonormal in $\ell^{2}(\mathbf{C})$, otherwise we can apply a Gram-Schmidt process and redefine the functions $G_{j}$. It is worth remarking that since the constraints are non-degenerated, such a process does not affect the regularity of the functions $G_{j}$ with respect to $\mathbf{c}$, once redefined.

4.1.4. Least action principle. The Action associated with the Lagrangian $\mathcal{L}$ over the time interval $[0, T]$ is the functional defined for all allowable shape function $\mathbf{c}:[0, T] \mapsto \mathcal{D}$ by:

$$
A(\mathbf{c}):=\int_{0}^{T} \mathcal{L}(\mathbf{c}(s), \dot{\mathbf{c}}(s)) \mathrm{ds}
$$

For any $\mathcal{C}^{1}$ function $\mathbf{c}:[0, T] \mapsto \mathcal{S}$, we define $\|\mathbf{c}\|_{\mathcal{C}^{1}([0, T], \mathcal{S})}:=\|\mathbf{c}\|_{L^{\infty}([0, T], \mathcal{S})}+\|\dot{\mathbf{c}}\|_{L^{\infty}([0, T], \mathcal{S})}$. An allowable curve $\mathbf{c}^{\dagger}:[0, T] \mapsto \mathcal{D}$ is a critical point of $A$ if it satisfies:

$$
A\left(\mathbf{c}^{\dagger}+\delta \mathbf{c}^{\dagger}\right)=A\left(\mathbf{c}^{\dagger}\right)+o\left(\left\|\delta \mathbf{c}^{\dagger}\right\|_{\mathcal{C}^{1}([0, T], \mathcal{S})}\right)
$$

for any perturbation $\delta \mathbf{c}^{\dagger}:[0, T] \mapsto \mathcal{S}$ such that $\delta \mathbf{c}^{\dagger}(0)=\delta \mathbf{c}^{\dagger}(T)=0$ and $\mathbf{c}^{\dagger}+\delta \mathbf{c}^{\dagger}$ be allowable. For any $\mathcal{C}^{1}$ function $\delta \mathbf{c}:[0, T] \mapsto \mathcal{S}$ such that $\delta \mathbf{c}(0)=\delta \mathbf{c}(T)=0$, the perturbation:

$$
\delta \mathbf{c}^{\dagger}:=\delta \mathbf{c}-\sum_{j \geq 1}\left(G_{j}\left(\mathbf{c}^{\dagger}\right), \delta \mathbf{c}\right)_{\ell^{2}(\mathbf{C})} G_{j}\left(\mathbf{c}^{\dagger}\right),
$$

meets with these requirements. We next compute that:

$$
A\left(\mathbf{c}^{\dagger}+\delta \mathbf{c}^{\dagger}\right)=A\left(\mathbf{c}^{\dagger}\right)+\int_{0}^{T}\left\langle\frac{\partial \mathcal{L}}{\partial \dot{\mathbf{c}}}\left(\mathbf{c}^{\dagger}\right), \delta \dot{\mathbf{c}}^{\dagger}\right\rangle+\left\langle\frac{\partial \mathcal{L}}{\partial \mathbf{c}}\left(\mathbf{c}^{\dagger}\right), \delta \mathbf{c}^{\dagger}\right\rangle \mathrm{d} s+o\left(\left\|\delta \mathbf{c}^{\dagger}\right\|_{\mathcal{C}^{1}([0, T], \mathcal{S})}\right) .
$$

Integrating by parts the main term in the right hand side, we classically get:

$$
\int_{0}^{T}\left\langle\frac{d}{d t} \frac{\partial \mathcal{L}}{\partial \dot{\mathbf{c}}}\left(\mathbf{c}^{\dagger}\right)-\frac{\partial \mathcal{L}}{\partial \mathbf{c}}\left(\mathbf{c}^{\dagger}\right), \delta \mathbf{c}^{\dagger}\right\rangle \mathrm{d} s=0
$$

Substituting the expression (4.5) of $\delta \mathbf{c}^{\dagger}$ into the identity above and taking into account that $\delta \mathbf{c}$ can be any $\mathcal{C}^{1}$ function $[0, T] \rightarrow \mathcal{S}$ vanishing at both extremities, we deduce the necessary condition for $\mathbf{c}^{\dagger}$ to be a critical point of $A$ :

$$
\left(\frac{d}{d t} \frac{\partial \mathcal{L}}{\partial \dot{\mathbf{c}}}\left(\mathbf{c}^{\dagger}\right)-\frac{\partial \mathcal{L}}{\partial \mathbf{c}}\left(\mathbf{c}^{\dagger}\right)\right)-\sum_{j=1}^{N^{\prime}} \lambda_{j}\left(\mathbf{c}^{\dagger}, \dot{\mathbf{c}}^{\dagger}\right) G_{j}\left(\mathbf{c}^{\dagger}\right)=0 \quad \text { in } \mathcal{S}^{\prime}
$$


where for any allowable shape function $\mathbf{c}, \lambda_{j}(\mathbf{c}, \dot{\mathbf{c}})(j \geq 1)$ are Lagrange's multipliers defined by:

$$
\lambda_{j}(\mathbf{c}, \dot{\mathbf{c}}):=\left\langle\frac{d}{d t} \frac{\partial \mathcal{L}}{\partial \dot{\mathbf{c}}}(\mathbf{c})-\frac{\partial \mathcal{L}}{\partial \mathbf{c}}(\mathbf{c}), G_{j}(\mathbf{c})\right\rangle .
$$

Equation (4.6) can next be further expanded. Since $\mathbb{K}(\mathbf{c}) \in \mathcal{L}_{2}(\mathcal{S} \times \mathcal{S})$, we deduce that, for all $\mathbf{c} \in \mathcal{D}$, $\partial \mathbb{K}(\mathbf{c}) / \partial \mathbf{c} \in \mathcal{L}_{3}(\mathcal{S} \times \mathcal{S} \times \mathcal{S})$ (see Appendix B), and the Frechet derivative of $\mathbb{K}$ in the direction of $\tilde{\mathbf{c}} \in \mathcal{S}$ at the point $\mathbf{c} \in \mathcal{D}$ is given by:

$$
\left\langle\frac{\partial \mathbb{K}}{\partial \mathbf{c}}(\mathbf{c}), \tilde{\mathbf{c}}, \cdot, \cdot\right\rangle \in \mathcal{L}_{2}(\mathcal{S} \times \mathcal{S}) .
$$

We then define the so-called Christoffel symbol $\Gamma(\mathbf{c}) \in \mathcal{L}_{3}(\mathcal{S} \times \mathcal{S} \times \mathcal{S})$ by:

$$
\left\langle\Gamma(\mathbf{c}), \tilde{\mathbf{c}}_{1}, \tilde{\mathbf{c}}_{2}, \tilde{\mathbf{c}}_{3}\right\rangle:=\frac{1}{2}\left[\left\langle\frac{\partial \mathbb{K}}{\partial \mathbf{c}}(\mathbf{c}), \tilde{\mathbf{c}}_{2}, \tilde{\mathbf{c}}_{1}, \tilde{\mathbf{c}}_{3}\right\rangle+\left\langle\frac{\partial \mathbb{K}}{\partial \mathbf{c}}(\mathbf{c}), \tilde{\mathbf{c}}_{2}, \tilde{\mathbf{c}}_{3}, \tilde{\mathbf{c}}_{1}\right\rangle-\left\langle\frac{\partial \mathbb{K}}{\partial \mathbf{c}}(\mathbf{c}), \tilde{\mathbf{c}}_{3}, \tilde{\mathbf{c}}_{1}, \tilde{\mathbf{c}}_{2}\right\rangle\right],
$$

for all $\tilde{\mathbf{c}}_{1}, \tilde{\mathbf{c}}_{2}, \tilde{\mathbf{c}}_{3} \in \mathcal{S}$. Notice that since the entries of the mass matrix $\mathbb{K}(\mathbf{c})$ can be explicitly computed using the formula of Subsection 3.2.5, the expression of $\Gamma(\mathbf{c})$, although quite complicated, can be explicitly computed as well.

Based on the identifications $\mathcal{L}_{2}(\mathcal{S} \times \mathcal{S})=\mathcal{L}\left(\mathcal{S}, \mathcal{S}^{\prime}\right)$ and $\mathcal{L}_{3}(\mathcal{S} \times \mathcal{S} \times \mathcal{S})=\mathcal{L}_{2}\left(\mathcal{S} \times \mathcal{S}, \mathcal{S}^{\prime}\right)$, we can consider $\mathbb{K}(\mathbf{c})$ as an element of $\mathcal{L}\left(\mathcal{S}, \mathcal{S}^{\prime}\right)$ and $\Gamma(\mathbf{c})$ as an element of $\mathcal{L}_{2}\left(\mathcal{S} \times \mathcal{S}, \mathcal{S}^{\prime}\right)$. Equation (4.6) can now be rewritten in the following form:

$$
\mathbb{K}(\mathbf{c}) \ddot{\mathbf{c}}+\langle\Gamma(\mathbf{c}), \dot{\mathbf{c}}, \dot{\mathbf{c}}\rangle+\frac{\partial W}{\partial \mathbf{c}}(\mathbf{c})=\sum_{j=1}^{N^{\prime}} \lambda_{j}(\mathbf{c}, \dot{\mathbf{c}}) G_{j}(\mathbf{c}) \quad \text { in } \mathcal{S}^{\prime}
$$

Taking into account the expressions (4.2), one notices that all of the terms of this equation can be explicitly computed. The well-posedness of this ODE relies on the invertibility of the operator $\mathbb{K}(\mathbf{c}): \mathcal{S} \rightarrow \mathcal{S}^{\prime}$ which is a quite involved question. Let us rather focus on the finite dimensional case.

4.2. Finite dimensional case. We consider now that the shape variable $\mathbf{c}$ lives in the finite $(N+1)$-dimensional space $\mathcal{S}_{N}$ (for some integer $N \geq 0$; see Appendix A) which can be identified, as well as its dual $\mathcal{S}_{N}^{\prime}$, with $\mathbf{C}^{N+1}$ or $\mathbf{R}^{2(N+1)}$. We denote $\mathcal{D}_{N}:=\mathcal{D} \cap \mathcal{S}_{N}$ and for any $\mu>0$, $\mathcal{A}_{N}(\mu):=\left\{\mathbf{c} \in \mathcal{S}_{N}: \sum_{k=-N}^{1} k\left|c_{k}\right|^{2}=\mu^{2}\right\}$ and $\mathcal{A}_{N}^{\bullet}(\mu):=\mathcal{A}_{N}(\mu) \cap \mathcal{D}_{N}$. The mass matrix $\mathbb{K}(\mathbf{c})$ turns out to be the actual $2(N+1) \times 2(N+1)$ symmetric matrix $\mathbb{K}(\mathbf{c}):=\mathbb{M}^{d}(\mathbf{c})-\mathbb{N}(\mathbf{c})^{T} \mathbb{M}^{r}(\mathbf{c})^{-1} \mathbb{N}(\mathbf{c})$ (the matrices $\mathbb{M}^{d}(\mathbf{c}), \mathbb{N}(\mathbf{c})$ and $\mathbb{M}^{r}(\mathbf{c})$ being defined in $\left.\S 3.2 .3\right)$ and equation (4.7) is set in $\mathbf{C}^{N+1}$.

First, let us prove that for some suitable values of $\varepsilon$ and $\alpha, W_{2}$ does the job it has been built for, namely:

Proposition 4.2. For any integer $N \geq 1$, any $\varepsilon>0$, any $\alpha \geq 1 / 2$ and any $\mathbf{c}^{*} \in \partial \mathcal{D}_{N}$, $W_{2}(\mathbf{c}) \rightarrow+\infty$ as $\mathbf{c} \rightarrow \mathbf{c}^{*}$.

Proof. For any $\mathbf{c} \in \mathcal{D}_{N}$, the functions $f(\mathbf{c}): \theta \in\left[0,2 \pi\left[\mapsto\left|c_{1}\right|^{2}-\left|\sum_{k>1} k c_{-k} e^{i k \theta}\right|^{2}\right.\right.$ are $2 \pi$ periodic, analytic and non-negative. Consider a sequence $\left(\mathbf{c}^{n}\right)_{n \geq 0} \subset \mathcal{D}_{N}$ such that $\mathbf{c}^{n} \rightarrow \mathbf{c}^{*} \in \partial \mathcal{D}_{N}$. The sequence of functions $\left(f\left(\mathbf{c}^{n}\right)\right)_{n \geq 0}$ converges uniformly to $f\left(\mathbf{c}^{*}\right)$ on $[0,2 \pi[$ and we draw the same conclusion for the functions $F_{n}:=\max _{j \geq n} f\left(\mathbf{c}^{j}\right)$. For any $\alpha>0$ and $\varepsilon>0$, the monotone convergence Theorem applies to the sequence of functions $\left(F_{n}^{-\alpha}\right)_{n \geq 0}$ valued in $[0,+\infty]$ and tells us that:

$$
\lim _{n \rightarrow+\infty} \int_{0}^{2 \pi} F\left(\mathbf{c}^{n}\right)(\theta)^{-\alpha} \mathrm{d} \theta=\int_{0}^{2 \pi} f\left(\mathbf{c}^{*}\right)(\theta)^{-\alpha} \mathrm{d} \theta .
$$


But $f\left(\mathbf{c}^{*}\right)$ is $2 \pi$-periodic, analytic and non-negative on $\left[0,2 \pi\left[\right.\right.$ and since $\mathbf{c}^{*} \notin \mathcal{D}_{N}$, there exists at least one $\theta_{0} \in\left[0,2 \pi\left[\right.\right.$ such that $f\left(\mathbf{c}^{*}\right)\left(\theta_{0}\right)=0$. From the non-negativity together with the regularity of $f\left(\mathbf{c}^{*}\right)$, we deduce that $f\left(\mathbf{c}^{*}\right)(\theta)=C\left(\theta-\theta_{0}\right)^{2}+o\left(\left(\theta-\theta_{0}\right)^{2}\right)$ in a neighborhood of the point $\theta=\theta_{0}$ for some constant $C \geq 0$ and hence that if $\alpha \geq 1 / 2$, the right hand side term in (4.8) is equal to $+\infty$. The proof is completed after noticing that, for all $n \geq 1$ :

$$
W_{2}\left(\mathbf{c}^{n}\right)=\varepsilon \int_{0}^{2 \pi} f\left(\mathbf{c}^{n}\right)(\theta)^{-\alpha} \mathrm{d} \theta \geq \varepsilon \int_{0}^{2 \pi} F\left(\mathbf{c}^{n}\right)(\theta)^{-\alpha} \mathrm{d} \theta .
$$

Second, we want to prove that the ODE (4.7) is well posed in $\mathbf{C}^{N+1}$. We need the following lemma:

Lemma 4.3. There exists a constant $\nu_{N}>0$ such that, for all $\mathbf{c} \in \mathcal{D}_{N}$ and all $\tilde{\mathbf{c}} \in \mathcal{S}_{N}$ :

$$
\tilde{\mathbf{c}}^{T} \mathbb{K}(\mathbf{c}) \tilde{\mathbf{c}} \geq \nu_{N}\|\tilde{\mathbf{c}}\|_{\ell^{2}(\mathbf{C})}^{2} .
$$

In particular, the matrix $\mathbb{K}(\mathbf{c})$ is invertible for all $\mathbf{c} \in \mathcal{D}_{N}$.

Proof. Let us set $X:=\left(-\mathbb{M}^{r}(\mathbf{c})^{-1} \mathbb{N}(\mathbf{c}) \tilde{\mathbf{c}}, \tilde{\mathbf{c}}\right)^{T} \in \mathcal{Q} \times \mathcal{S}_{N}$ and observe that:

$$
X^{T} \mathbb{M}(\mathbf{c}) X=\tilde{\mathbf{c}}^{T} \mathbb{K}(\mathbf{c}) \tilde{\mathbf{c}},
$$

where the matrix $\mathbb{M}(\mathbf{c})$ is defined in (3.14). We next easily get that, since $\mathbb{M}^{r}(\mathbf{c})$ is positive:

$$
\begin{aligned}
X^{T} \mathbb{M}(\mathbf{c}) X & \geq \frac{1}{2}\left[\mathbb{M}^{r}(\mathbf{c})^{-1} \mathbb{N}(\mathbf{c}) \tilde{\mathbf{c}}\right]^{T} \mathbb{M}^{r}(\mathbf{c})\left[\mathbb{M}^{r}(\mathbf{c})^{-1} \mathbb{N}(\mathbf{c}) \tilde{\mathbf{c}}\right]+\frac{1}{2} \tilde{\mathbf{c}}^{T} \mathbb{M}^{d}(\mathbf{c}) \tilde{\mathbf{c}} \\
& \geq \frac{1}{2} \tilde{\mathbf{c}}^{T} \mathbb{M}^{d}(\mathbf{c}) \tilde{\mathbf{c}} \geq \frac{1}{2} \pi \varrho_{0} \sum_{k=-N}^{1} \frac{1}{|k|+1}\left(\tilde{a}_{k}^{2}+\tilde{b}_{k}^{2}\right),
\end{aligned}
$$

where $\tilde{c}_{k}=\tilde{a}_{k}+i \tilde{b}_{k}$ for all $k \in\{-N, \ldots,-1,1\}$. The proof is completed after setting $\nu_{N}:=$ $\varrho_{0} \pi /(2(N+1))$.

REMARK 4.4. Observe that the conclusion of this lemma is no longer true in the general infinite dimensional case since $\nu_{N} \rightarrow 0$ as $N \rightarrow \infty$.

This lemma allows us the rewrite the ODE (4.7) in the normal form:

$$
\frac{d}{d t}\left[\begin{array}{l}
\dot{\mathbf{c}} \\
\mathbf{c}
\end{array}\right]=\left[\begin{array}{c}
\Lambda(\mathbf{c}, \dot{\mathbf{c}})+\sum_{j=1}^{N^{\prime}} \lambda_{j}(\mathbf{c}, \dot{\mathbf{c}}) \mathbb{K}(\mathbf{c})^{-1} G_{j}(\mathbf{c}) \\
\dot{\mathbf{c}}
\end{array}\right],
$$

where

$$
\Lambda(\mathbf{c}, \dot{\mathbf{c}}):=-\mathbb{K}(\mathbf{c})^{-1}\langle\Gamma(\mathbf{c}), \dot{\mathbf{c}}, \dot{\mathbf{c}}\rangle-\mathbb{K}(\mathbf{c})^{-1} \frac{\partial W}{\partial \mathbf{c}}(\mathbf{c}) .
$$

The expressions of the Lagrange's multipliers $\lambda_{j}(\mathbf{c}, \dot{\mathbf{c}})\left(1 \leq j \leq N^{\prime}\right)$ can be somehow clarified. Differentiating with respect to the time the identity $\left(G_{j}(\mathbf{c}), \dot{\mathbf{c}}\right)_{\ell^{2}(\mathbf{C})}=0$, we obtain:

$$
\left(\left\langle\frac{\partial G_{j}}{\partial \mathbf{c}}, \dot{\mathbf{c}}\right\rangle, \dot{\mathbf{c}}\right)_{\ell^{2}(\mathbf{C}}+\left(G_{j}(\mathbf{c}), \ddot{\mathbf{c}}\right)_{\ell^{2}(\mathbf{C})}=0 .
$$


Taking into account the ODE satisfied by $\ddot{\mathbf{c}}$, we obtain that, for all $1 \leq j \leq N^{\prime}$ :

$$
\left(\left\langle\frac{\partial G_{j}}{\partial \mathbf{c}}, \dot{\mathbf{c}}\right\rangle, \dot{\mathbf{c}}\right)_{\ell^{2}(\mathbf{C})}+\sum_{k=1}^{N^{\prime}} \lambda_{k}(\mathbf{c}, \dot{\mathbf{c}})\left(G_{j}(\mathbf{c}), \mathbb{K}(\mathbf{c})^{-1} G_{k}(\mathbf{c})\right)_{\ell^{2}(\mathbf{C})}+\left(G_{j}(\mathbf{c}), \Lambda(\mathbf{c}, \dot{\mathbf{c}})\right)_{\ell^{2}(\mathbf{C})}=0
$$

and this system of equations can be rewritten in the form:

$$
\begin{aligned}
& {\left[\begin{array}{ccc}
\left(G_{1}(\mathbf{c}), \mathbb{K}(\mathbf{c})^{-1} G_{1}(\mathbf{c})\right)_{\ell^{2}(\mathbf{C})} & \cdots & \left(G_{1}(\mathbf{c}), \mathbb{K}(\mathbf{c})^{-1} G_{N^{\prime}}(\mathbf{c})\right)_{\ell^{2}(\mathbf{C})} \\
\vdots & \vdots & \\
\left(G_{N^{\prime}}(\mathbf{c}), \mathbb{K}(\mathbf{c})^{-1} G_{1}(\mathbf{c})\right)_{\ell^{2}(\mathbf{C})} & \cdots & \left(G_{N^{\prime}}(\mathbf{c}), \mathbb{K}(\mathbf{c})^{-1} G_{N^{\prime}}(\mathbf{c})\right)_{\ell^{2}(\mathbf{C})}
\end{array}\right]\left[\begin{array}{c}
\lambda_{1}(\mathbf{c}, \dot{\mathbf{c}}) \\
\vdots \\
\lambda_{N^{\prime}}(\mathbf{c}, \dot{\mathbf{c}})
\end{array}\right]=} \\
& {\left[\begin{array}{c}
-\left(G_{1}(\mathbf{c}), \Lambda(\mathbf{c}, \dot{\mathbf{c}})\right)_{\ell^{2}(\mathbf{C})}-\left(\left\langle\frac{\partial G_{1}}{\partial \mathbf{c}}, \dot{\mathbf{c}}\right\rangle, \dot{\mathbf{c}}\right)_{\ell^{2}(\mathbf{C})} \\
\vdots \\
-\left(G_{N^{\prime}}(\mathbf{c}), \Lambda(\mathbf{c}, \dot{\mathbf{c}})\right)_{\ell^{2}(\mathbf{C})}-\left(\left\langle\frac{\partial G_{N^{\prime}}}{\partial \mathbf{c}}, \dot{\mathbf{c}}\right\rangle, \dot{\mathbf{c}}\right)_{\ell^{2}(\mathbf{C})}
\end{array}\right] .}
\end{aligned}
$$

The matrix $\mathbb{K}(\mathbf{c})$ is symmetric and according to Lemma 4.9 it is definite positive. We draw the same conclusion first for $\mathbb{K}(\mathbf{c})^{-1}$ and next for the square matrix on the left hand side of (4.12). The linear system (4.12) is hence invertible for all $\mathbf{c} \in \mathcal{S}$ and the Lagrange's multipliers $\lambda_{j}(\mathbf{c}, \dot{\mathbf{c}})$ are well-defined and smooth in the variables $\mathbf{c}$ and $\dot{\mathbf{c}}$. We can now state:

Theorem 4.5. For any integer $N \geq 1$, for any $\mu>0$ and for any initial data $\left(\mathbf{c}_{0}, \dot{\mathbf{c}}_{0}\right) \in$ $\mathcal{A}_{N}^{\bullet}(\mu) \times \mathcal{S}_{N}$ satisfying the compatibility conditions $\left(G_{j}\left(\mathbf{c}_{0}\right), \dot{\mathbf{c}}_{0}\right)_{\ell^{2}(\mathbf{C})}=0$ for all $1 \leq j \leq N^{\prime}$, there exists a unique $\mathcal{C}^{2}$ solution $t \in\left[0,+\infty\left[\mapsto \mathbf{c}(t) \in \mathcal{A}_{N}(\mu)\right.\right.$ to the $O D E$ (4.10) satisfying $\mathbf{c}(0)=\mathbf{c}_{0}$, $\dot{\mathbf{c}}(0)=\dot{\mathbf{c}}_{0}$ and for all time $\left.t \in\right] 0,+\infty\left[\right.$, the constraints $\left(G_{j}(\mathbf{c}(t)), \dot{\mathbf{c}}(t)\right)_{\ell^{2}(\mathbf{C})}=0$ for all $1 \leq j \leq N^{\prime}$. Besides, if $\varepsilon>0$ and $\alpha \geq 1 / 2$ in the definition of $W_{2}$ (see Proposition 4.2) the solution is allowable (in the sense of Definition 2.2) and analytic if the functions $V$ and $G_{j}$ are.

Proof. Remark that any solution of (4.10) satisfies the identities (4.11) for all time. Hence, if the initial data $\left(\mathbf{c}_{0}, \dot{\mathbf{c}}_{0}\right)$ satisfies the constraints at the initial time, the corresponding solution will still satisfy them for all time $t>0$. In particular, if $\mathbf{c}_{0} \in \mathcal{A}_{N}(\mu)$, the solution will remain in $\mathcal{A}_{N}(\mu)$ for all time $t>0$.

All of the terms in the right hand side of equation (4.10) are analytic with respect to $(\mathbf{c}, \dot{\mathbf{c}}) \in$ $\mathcal{D}_{N} \times \mathcal{S}_{N}$. The Cauchy-Lipschitz Theorem applies and ensures the existence of a unique maximal analytic solution defined on a time interval $[0, T)$ for some $T>0$. Recall now that an equivalent formulation of ODE (4.10) is:

$$
\left(\frac{d}{d t} \frac{\partial \mathcal{L}}{\partial \dot{\mathbf{c}}}(\mathbf{c})-\frac{\partial \mathcal{L}}{\partial \mathbf{c}}(\mathbf{c})\right)-\sum_{j=1}^{N^{\prime}} \lambda_{j}(\mathbf{c}, \dot{\mathbf{c}}) G_{j}(\mathbf{c})=0,
$$

and taking the scalar product in $\ell^{2}(\mathbf{C})$ with $\dot{\mathbf{c}}$, we get, since $\left(G_{j}(\mathbf{c}), \dot{\mathbf{c}}\right)_{\ell^{2}(\mathbf{C})}=0$ for all $\left.\left.t \in\right] 0, T\right)$ :

$$
\left(\frac{d}{d t} \frac{\partial \mathcal{L}}{\partial \dot{\mathbf{c}}}(\mathbf{c})-\frac{\partial \mathcal{L}}{\partial \mathbf{c}}(\mathbf{c}), \dot{\mathbf{c}}\right)_{\ell^{2}(\mathbf{C})}=0
$$

If we introduce now the overall energy $E=(\mathbb{K}(\mathbf{c}) \dot{\mathbf{c}}, \dot{\mathbf{c}})_{\ell^{2}(\mathbf{C})} / 2+W(\mathbf{c})$, we easily obtain that

$$
\frac{d}{d t} E=\frac{d}{d t}\left(\left(\frac{\partial \mathcal{L}}{\partial \dot{\mathbf{c}}}, \dot{\mathbf{c}}\right)_{\ell^{2}(\mathbf{C})}-\mathcal{L}\right)=\left(\frac{d}{d t} \frac{\partial \mathcal{L}}{\partial \dot{\mathbf{c}}}(\mathbf{c})-\frac{\partial \mathcal{L}}{\partial \mathbf{c}}(\mathbf{c}), \dot{\mathbf{c}}\right)_{\ell^{2}(\mathbf{C})}=0,
$$


and not surprisingly, the energy is conserved. It entails, according to Proposition 4.2, that the solution remains in $\mathcal{D}_{N}$ for all $t \in[0, T)$ and hence in $\mathcal{A}_{N}^{\bullet}(\mu)$. According to Lemma 4.3 , we deduce also that $\nu_{N}\|\dot{\mathbf{c}}\|_{\ell^{2}(\mathbf{C})}^{2} \leq 2 E(0)$ and the solution cannot blow up in finite time. Eventually, classical results on the behavior of solutions of ODEs ensure us that the solution can be indefinitely continued.

4.2.1. Damping effects. The larger $N$ is, the higher the frequencies of the oscillatory elastic motion of the body are, making the numerical integration of ODE (4.10) a quite stiff problem. This problem can be overcome by adding a damping term in the right hand side of ODE (4.13), turning it into:

$$
\left(\frac{d}{d t} \frac{\partial \mathcal{L}}{\partial \dot{\mathbf{c}}}(\mathbf{c})-\frac{\partial \mathcal{L}}{\partial \mathbf{c}}(\mathbf{c})\right)-\sum_{j=1}^{N^{\prime}} \lambda_{j}(\mathbf{c}, \dot{\mathbf{c}}) G_{j}(\mathbf{c})=-\mathbb{D} \dot{\mathbf{c}}, \quad(t>0)
$$

where $\mathbb{D}$ is a square symmetric positive matrix. Regarding the normal form (4.10) of the ODE, we only have to modify the expression $(4.10 \mathrm{~b})$ of $\Lambda(\mathbf{c}, \dot{\mathbf{c}})$ as follows:

$$
\Lambda(\mathbf{c}, \dot{\mathbf{c}}):=-\mathbb{K}(\mathbf{c})^{-1}\langle\Gamma(\mathbf{c}), \dot{\mathbf{c}}, \dot{\mathbf{c}}\rangle-\mathbb{K}(\mathbf{c})^{-1} \frac{\partial W}{\partial \mathbf{c}}(\mathbf{c})-\mathbb{K}(\mathbf{c})^{-1} \mathbb{D} \dot{\mathbf{c}}
$$

Notice that the conclusion of Theorem 4.5 still holds, the only modification in the proof consisting in replacing the equality (4.14) by the energy estimate:

$$
\frac{d}{d t} E=\left(\frac{d}{d t} \frac{\partial \mathcal{L}}{\partial \dot{\mathbf{c}}}(\mathbf{c})-\frac{\partial \mathcal{L}}{\partial \mathbf{c}}(\mathbf{c}), \dot{\mathbf{c}}\right)_{\ell^{2}(\mathbf{C})}=-(\mathbb{D} \dot{\mathbf{c}}, \dot{\mathbf{c}})_{\ell^{2}(\mathbf{C})} \leq 0 .
$$

4.2.2. Locked variables. We may wish to lock some components of the shape variable. From a mechanical point of view, it means that the body is rigid with respect to these components. This can be easily expressed with the constraints $G_{j}$ and by choosing properly the initial conditions, as stated in Theorem 4.5. Suppose for instance that we want the real part $a_{m}$ of the component $c_{m}$ (for some $1 \leq m \leq N)$ to remain equal to its initial value for all times. It suffices to introduce the constant function $G_{3}: \mathbf{c} \in \mathbf{C}^{(N+1)} \mapsto \mathbf{C}^{(N+1)}$ such that $G_{3}(\mathbf{c})=(0, \ldots, 0,1,0, \ldots, 0)$ where 1 is at the $m$-th position and to set the initial velocity $\dot{\mathbf{c}}_{0}:=\left(\dot{a}_{k}^{0}+i \dot{b}_{k}^{0}\right)_{1 \leq k \leq N}$ such that $\dot{a}_{m}^{0}=0$ (i.e. $\left.\left(G_{3}\left(\mathbf{c}_{0}\right), \dot{\mathbf{c}_{0}}\right)_{\ell^{2}(\mathbf{C})}=0\right)$.

4.2.3. Degrees of freedom. The number of degrees of freedom of the overall fluid-swimmer system is also the number of unknowns to be computed. It is equal to $\mathrm{d}_{\text {free }}=2(N+1)-N^{\prime}+3=$ $2 N-N^{\prime}+5$. Indeed, there are $2(N+1)$ degrees of freedom for the deformations of the body, $N^{\prime}$ is the number of independent constraints to which we add the three degrees of freedom relating to the rigid motion of the swimmer.

4.3. Numerical simulations. The numerical scheme is straightforward. We use the explicit expressions of the mass matrices given in Subsection 3.2.5. Although quite involved, the expression of the Christoffel symbol $\Gamma$ can be computed explicitly as well (using for instance a symbolic calculator). To integrate the ODE (4.10), we use the built-in MATLAB solver ode113 (which is a variable order Adams-Bashforth-Moulton PECE solver; we refer to the MATLAB documentation for further details).

The numerics of this paper has to be read with the web page http://www.iecn.u-nancy.fr/ munnier/passive_locomotion/index.html, which contains all of the simulations and additional material. 
In all of the following examples, the function $V$ in the definition of the elastic potential energy has the form: $V(\mathbf{c}):=\mathbf{c}^{T} \mathbb{R} \mathbf{c}$ where $\mathbb{R}$ is a diagonal positive matrix, called the rigidity matrix. We set $N=7$ so $\mathbf{c}:=\left(a_{1}+i b_{1}, a_{-1}+i b_{-1}, \ldots, a_{-7}+i b_{-7}\right) \in \mathbf{C}^{8}$ (there are, at most, 16 degrees of freedom relating to the deformations. Some of them can be locked in some examples).

We will mainly address the following questions:

1. How to achieve passive locomotion (i.e. locomotion at zero energy cost)?

2. The fluid-swimmer system is conservative. What is the time evolution of the distribution of energy, within the system, between the kinetic energy of the body, the kinetic energy of the fluid and the potential energy of elasticity?

3. Locomotion results from to the exchange of impulse between the shape changes and the fluid. As an isolated system, the overall impulse of the fluid-swimmer system is conserved. What is the time evolution of the distribution of impulse within the system?

Example 1: Passive locomotion of a shape changing, non-elastic swimmer. In this first example, we show that quite surprisingly, the swimmer does not even have to be elastic to experience passive locomotion. The body is deformable but does not tend to recover any prescribed shape. Even without any potential energy of elasticity, the overall fluid-swimmer system still naturally behaves like an oscillating mechanical system. With suitable initial data, the shape variable, solution to ODE (4.10), is time-periodic and the relating shape changes are periodic strokes that make the body swim. We will show that this phenomenon is possible even when the initial velocity of the body is null to prove that the swimming is not a consequence of D'Alembert's paradox.

We first consider that all of the components of $\mathbf{c}$ are fixed, excepted $c_{-1}=a_{-1}+i b_{-1}$ and $c_{-2}=a_{-2}+i b_{-2}$. So $N^{\prime}=14$ and $\mathrm{d}_{\text {free }}=2 N-N^{\prime}+5=5$ (in particular, there are only 2 degrees of freedom relating to the shape changes). All of the data are made explicit in the table below. We use the symbol $*$ for unused quantities. The second row of the table gives the general form of the shape variable $\mathbf{c}$, and in particular the values of the locked components.

\begin{tabular}{|c|c|c|c|}
\hline $\mathrm{d}_{\text {free }}=5$ & $\varrho_{0}=1$ & $\alpha=*$ & $\varepsilon=0$ \\
\hline \multicolumn{4}{|c|}{$\mathbf{c}=\left(1,0, a_{-1}, b_{-1}, a_{-2}, b_{-2}, 0, \ldots, 0\right)$} \\
\hline \multicolumn{4}{|c|}{$\bar{R}=0$ (no potential energy of elasticity) } \\
\hline \multicolumn{4}{|c|}{$\mathbb{D}=0$ (no damping) } \\
\hline \multicolumn{4}{|l|}{$\mathbf{c}_{\mathrm{ref}}=*$} \\
\hline \multicolumn{4}{|c|}{$\mathbf{c}_{0}=\left(1, a_{-1}^{0}, b_{-1}^{0}, a_{-2}^{0}, b_{-2}^{0}, 0, \ldots, 0\right)$} \\
\hline \multicolumn{4}{|c|}{$\dot{\mathbf{c}}_{0}=\left(0, \dot{a}_{-1}^{0}, \dot{b}_{-1}^{0}, \dot{a}_{-2}^{0}, \dot{b}_{-2}^{0}, 0, \ldots, 0\right)$} \\
\hline
\end{tabular}

Let us recall that the initial data have to satisfy the compatibility conditions: $\dot{a}_{-1}^{0} a_{-1}^{0}+\dot{b}_{-1}^{0} b_{-1}^{0}+$ $2 \dot{a}_{-2}^{0} a_{-2}^{0}+2 \dot{b}_{-2}^{0} b_{-2}^{0}=0$ (conservation of the body's volume) and $\left(\dot{b}_{-1}^{0} a_{-1}^{0}-\dot{a}_{-1}^{0} b_{-1}^{0}\right) / 2+\left(\dot{b}_{-2}^{0} a_{-2}^{0}-\right.$ $\left.\dot{a}_{-2}^{0} b_{-2}^{0}\right) / 3=0$ (deformations resulting from interior forces only). When $\dot{b}_{-1}^{0}=\dot{a}_{-2}^{0}=0$, we seek a particular solution to ODE (4.10) in the form

$$
\mathbf{c}(t):=(1, \gamma \cos (\beta(t)), 0,0, \gamma \sin (\beta(t)) / \sqrt{2}, 0, \ldots, 0) .
$$

We obtain that the constant $\gamma$ has to be equal to $\sqrt{\left|a_{-1}^{0}\right|^{2}+2\left|b_{-2}^{0}\right|^{2}}$, and the function $\beta: \mathbf{R} \rightarrow \mathbf{R} / 2 \pi$ has to satisfy a second order autonomous ODE (see the phase portrait on Fig. 4.1) with initial data $\left(\beta_{0}, \dot{\beta}_{0}\right)$ satisfying $\cos \left(\beta_{0}\right)=a_{-1}^{0} / \gamma, \sin \left(\beta_{0}\right)=b_{-2}^{0} \sqrt{2} / \gamma$ and $\dot{\beta}_{0}=\sqrt{2}\left(\dot{b}_{-2}^{0} a_{-1}^{0}-\dot{a}_{-1}^{0} b_{-2}^{0}\right) / \gamma^{2}$. One can observe on Fig. 4.1 that any solution $t \mapsto \beta(t) \in \mathbf{R} / 2 \pi$ is time periodic (the period depending on the initial data) and consequently, any relating solution (4.17) is also time-periodic. We can 
next plug the function $t \mapsto \mathbf{c}(t)$ into the ODE (3.21) to compute the rigid displacement of the body in the fluid. We obtain that any (non-zero) solution yields a net displacement of the body along the $y$-axis (see Fig. 4.2 and Fig. 4.3). For any solutions, the time evolution of the distribution of energy is periodic (see Fig. 4.4). Moreover, there exists always an instant $t^{\dagger}$ at which the kinetic energy of the body relating to its rigid motion is null (see the dashed straight line on Fig. 4.4). At this time, the rigid velocity of the body is null as well. If we choose the values of $\mathbf{c}\left(t^{\dagger}\right)$ and $\dot{\mathbf{c}}\left(t^{\dagger}\right)$ as Cauchy data for the second order ODE (4.10), we will obtain the same periodic solution but $t^{\dagger}$ time-shifted. Furthermore, the corresponding initial (rigid) velocity of the body will be zero. This proves that passive locomotion, starting at zero velocity, is possible as well and that the net displacement is not a pure consequence of D'Alembert's paradox (the displacement does not result from a non-zero initial impulse) but can be seen as a generalization, to deformable bodies, of this phenomenon.

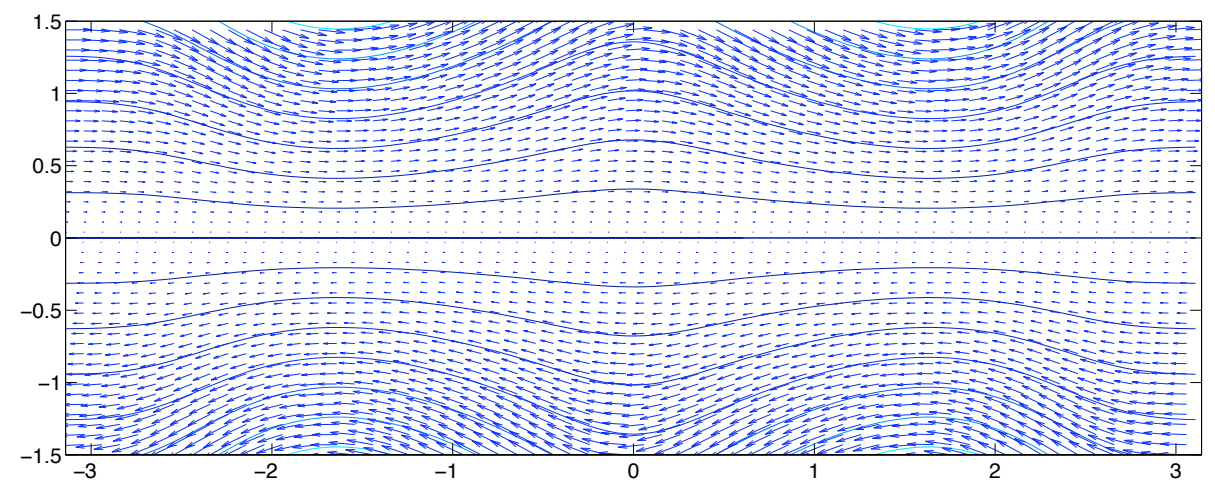

FIG. 4.1. Phase portrait of the ODE solved by the function $\beta$, introduced in Example 1. The x-coordinate is the value of $\beta$, the $y$-coordinate the value of $\dot{\beta}$ and the arrows are vectors of coordinates $(\ddot{\beta}, \dot{\beta})$. The lines are some solutions $(\beta, \dot{\beta})$ to the ODE (and are also some level sets of the overall energy of the fluid-swimmer system). Although there is no elasticity in this model, the fluid-swimmer system is oscillating. Any solution $\beta: \mathbf{R} \rightarrow \mathbf{R} / 2 \pi$ is time periodic and ranges from $-\pi$ to $\pi$. Once substituted in the ODE (3.21), any solution produces periodic strokes and a net rigid displacement of the swimmer as pictured on Fig. 4.3.

Example 2: Passive locomotion of an elastic swimmer. We consider now that the body is hyperelastic and tends to recover a given shape $\mathbf{c}_{\mathrm{ref}}$ minimizing the potential energy $W_{1}(\mathbf{c}):=$ $\left(\mathbf{c}-\mathbf{c}_{\mathrm{ref}}\right)^{T} \mathbb{R}\left(\mathbf{c}-\mathbf{c}_{\mathrm{ref}}\right)$. Like in the preceding example, all of the components of $\mathbf{c}$ are fixed, excepted $c_{-1}$ and $c_{-2}$. More precisely, we consider the following data:

\begin{tabular}{|c|c|c|c|}
\hline $\mathrm{d}_{\text {free }}=5$ & $\varrho_{0}=1$ & $\alpha=*$ & $\varepsilon=0$ \\
\hline \multicolumn{4}{|c|}{$\mathbf{c}=\left(1, a_{-1}, b_{-1}, a_{-2}, b_{-2}, 0, \ldots, 0\right)$} \\
\hline \multicolumn{4}{|c|}{$\mathbb{R}=\operatorname{diag}\left(0,0, \kappa_{3}, \kappa_{4}, \kappa_{5}, \kappa_{6}, 0, \ldots, 0\right)$} \\
\hline \multicolumn{4}{|c|}{$\mathbb{D}=0$ (no damping) } \\
\hline \multicolumn{4}{|c|}{$\mathbf{c}_{\mathrm{ref}}=\left(1, a_{-1}^{r}, b_{-1}^{r}, a_{-2}^{r}, b_{-2}^{r}, 0, \ldots, 0\right)$} \\
\hline \multicolumn{4}{|c|}{$\mathbf{c}_{0}=\left(1, a_{-1}^{0}, b_{-1}^{0}, a_{-2}^{0}, b_{-2}^{0}, 0, \ldots, 0\right)$} \\
\hline \multicolumn{4}{|c|}{$\dot{\mathbf{c}}_{0}=\left(0, \dot{a}_{-1}^{0}, \dot{b}_{-1}^{0}, \dot{a}_{-2}^{0}, \dot{b}_{-2}^{0}, 0, \ldots, 0\right)$} \\
\hline
\end{tabular}

In the definition of $\mathbb{R}$, the components $\kappa_{j}(j=3,4,5,6)$ are non-negative real numbers. When $\dot{b}_{-1}^{0}=\dot{a}_{-2}^{0}=0$, the particular form (4.17) of the shape variable still yields a particular solution to the ODE (4.10), providing that $\beta$ solves a second order autonomous ODE, of which some examples 


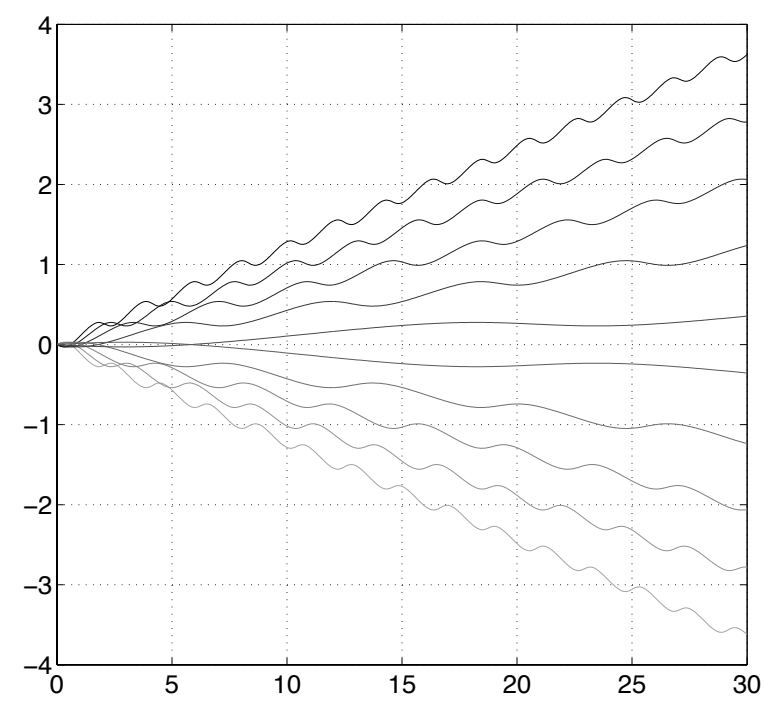

FIG. 4.2. The shape changes resulting from the use of the function $\beta$ (from Example 1) in ODE (3.21) are periodic strokes that produce a displacement of the body along the $y$-axis. In this figure is plotted the $y$-coordinate of the center of mass of the body with respect to the time for different initial data. For all of the curves, $\gamma=0.5$ and $\beta_{0}=0$. The initial velocity is, from the bottom to the top, $\dot{\beta}_{0}=2,1.55,1.1,0.65,0.2,-0.2,-0.65,-1.1,-1.55,-2$.

of phase portraits are given in Fig. 4.5, 4.6 and 4.7 for different rigidity matrices $\mathbb{R}$ and different reference shapes $\mathbf{c}_{\text {ref }}$. All of the solutions $\beta: \mathbf{R} \rightarrow \mathbf{R} / 2 \pi$ are still periodic but not all of them range from $-\pi$ to $\pi$. Indeed, one or several wells of potential appear. If the initial data $\left(\beta_{0}, \dot{\beta}_{0}\right)$ is too close to such a well, the solution will remain for all times in its vicinity. The relating shape changes turn out to be a flapping motion and when substituted into the ODE (3.21), such a trapped solution does not result in locomotion but only in a reciprocal motion. This flapping phenomenon is addressed in the so-called Purcell's scallop theorem (stated primarily in [24] for low Reynolds numbers swimmers and recently extended to high Reynolds numbers swimmers in [6]).

All of the solutions that are not trapped in a potential well are quite similar to the solutions obtained in the first example and result in passive locomotion. Such a solution will be thoroughly studied in the following example.

Example 3: Passive locomotion and distributions of energy and impulse. In this example, we are interested in studying the time evolution of the distribution of energy and impulse for a particular case of passive locomotion. The impulse is a momentum like quantity already defined in Subsection 3.3. The impulse of the fluid-swimmer system is conserved and always finite whereas the momentum is usually infinite. As usual, the data we chose are detailed in the table below. As in the preceding examples, only the components $a_{-1}$ and $b_{-2}$ play a role. The reference configuration (i.e. minimizing the potential energy) is an ellipse. 


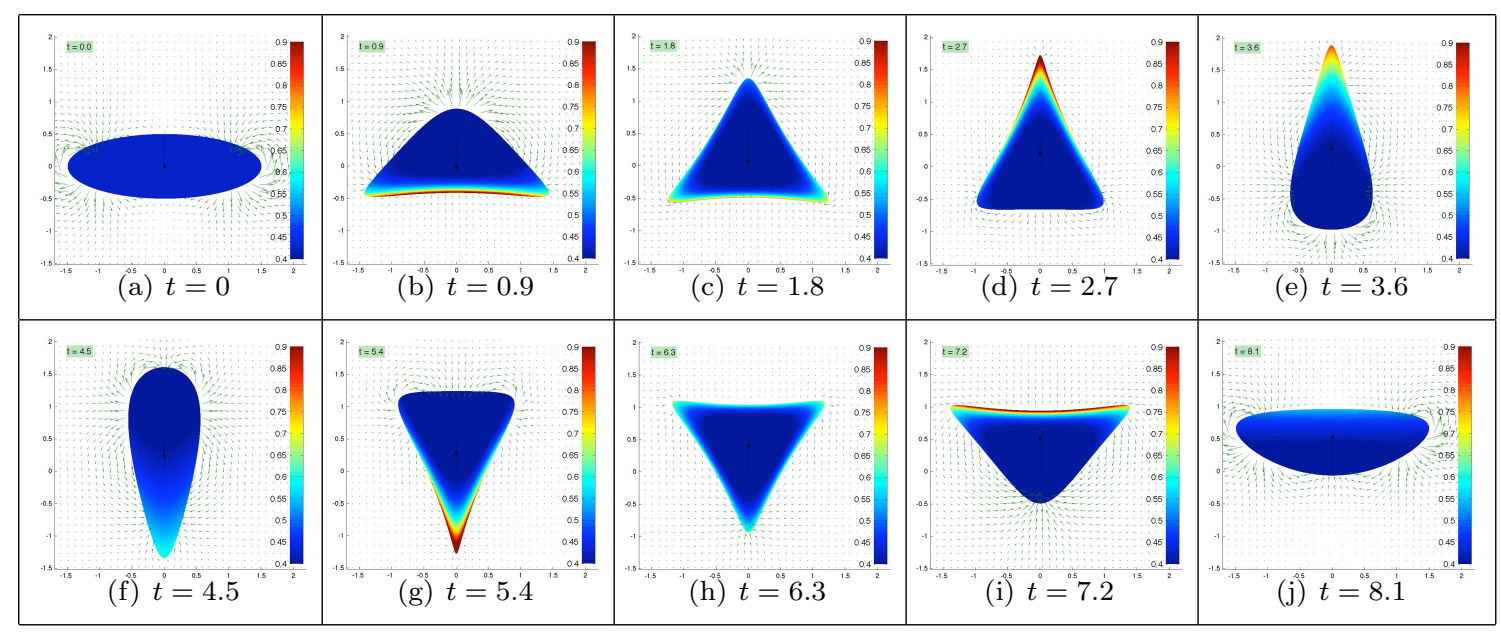

FIG. 4.3. Screenshots of the swimmer (as described in Example 1) over a stroke. Here $\gamma=0.5$ and the initial data are $\beta_{0}=0$ and $\dot{\beta}_{0}=-1$. The black spot shows the position of the center of mass and the dashed black line is its trajectory. The colors give the value of the internal density. As predicted by the maximum principle (see equalities (4.1) and the explanation therein), compression occurs mainly along the boundary. At last, the arrows represent the Eulerian velocity of the fluid (the animation can be found on the web page whose the location is given at the beginning of this subsection).

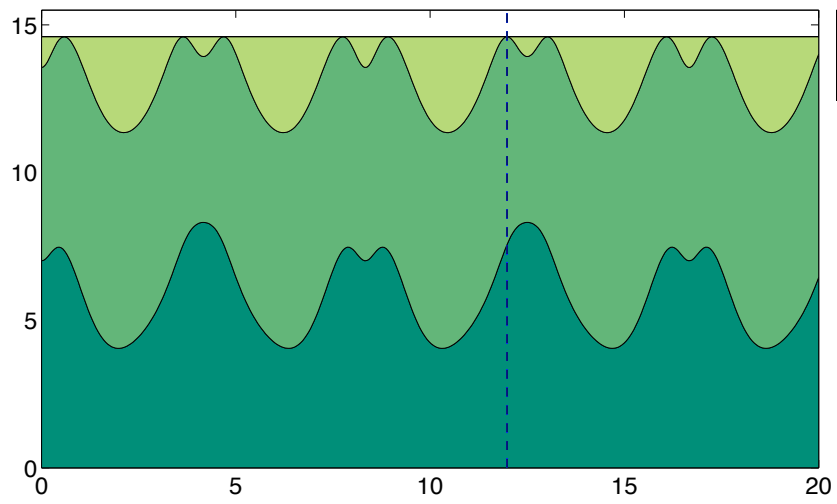

Kinetic energy of the fluid

Kinetic energy of the body (deformations)

Kinetic energy of the body (rigid motion)

FIG. 4.4. Distribution of energy for the example corresponding to the data $\gamma=0.5, \beta_{0}=0$ and $\dot{\beta}_{0}=-1$ (Example 1, screenshots on Fig. 4.3). The fluid-body system is conservative, so the overall energy is constant. It can be decomposed at any time into the kinetic energy of the fluid and the kinetic energy of the body. The latter can be decomposed into the kinetic energy resulting from the shape changes and the kinetic energy of the rigid motion. In this graphic, the amount of each type of energy is proportional to the area. The dashed line corresponds to a time at which the rigid velocity of the swimmer is null.

\begin{tabular}{|c|c|c|c|}
\hline $\mathrm{d}_{\text {free }}=5$ & $\varrho_{0}=1$ & $\alpha=*$ & $\varepsilon=0$ \\
\hline \multicolumn{4}{|c|}{$\mathbf{c}=\left(1, a_{-1}, b_{-1}, a_{-2}, b_{-2}, 0, \ldots, 0\right)$} \\
\hline \multicolumn{4}{|c|}{$\mathbb{R}=\operatorname{diag}(0,0,1,1,2,2,0, \ldots, 0)$} \\
\hline \multicolumn{4}{|c|}{$\mathbb{D}=0$ (no damping $)$} \\
\hline \multicolumn{4}{|c|}{$\mathbf{c}_{\mathrm{ref}}=(0,0,0.4899,0, \ldots, 0)$} \\
\hline \multicolumn{4}{|c|}{$\mathbf{c}_{0}=(0,0,0.4,0,0,0.2,0, \ldots, 0)$} \\
\hline \multicolumn{4}{|c|}{$\dot{\mathbf{c}}_{0}=(0,0,0.6,0,0,-0,6,0, \ldots, 0)$} \\
\hline
\end{tabular}




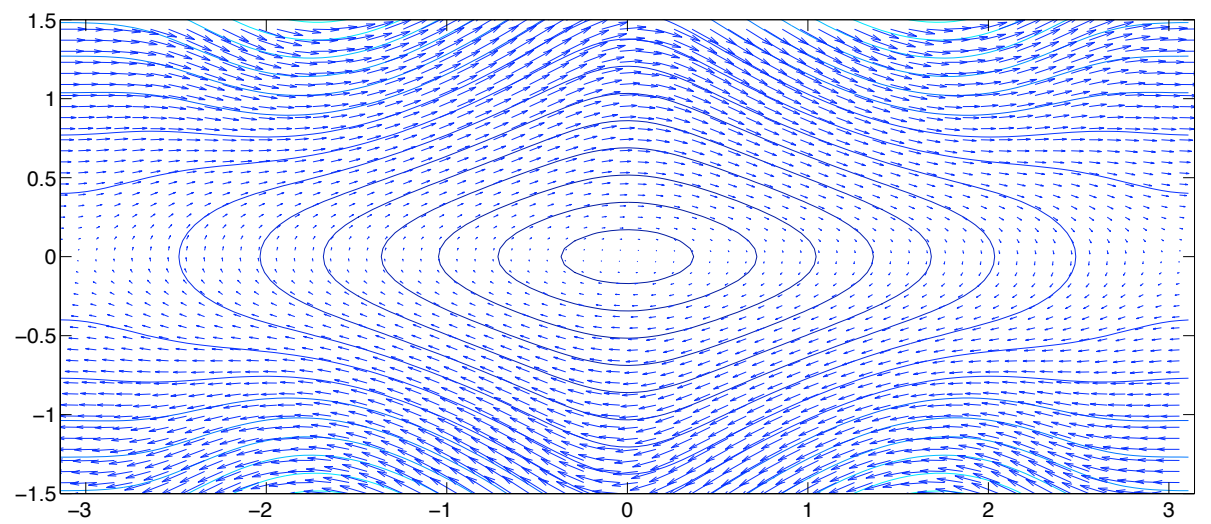

FIG. 4.5. This phase portrait of the ODE solved by $\beta$ (from Example 2) is obtained with $\gamma=0.5$ and $\kappa_{j}=0.5$ $(j=3,4,5,6)$ (all of the the components of the rigidity matrix are equal to 0.5$)$. The reference configuration minimizing the potential energy of elasticity is an ellipse whose the shape variable is $\mathbf{c}_{\mathrm{ref}}=(1,0, \gamma, 0, \ldots, 0)$ corresponding to $\beta=0$ when put in the form (4.17). So, not surprisingly, the center of the well of potential is centered at the point $\beta=0, \dot{\beta}=0$.

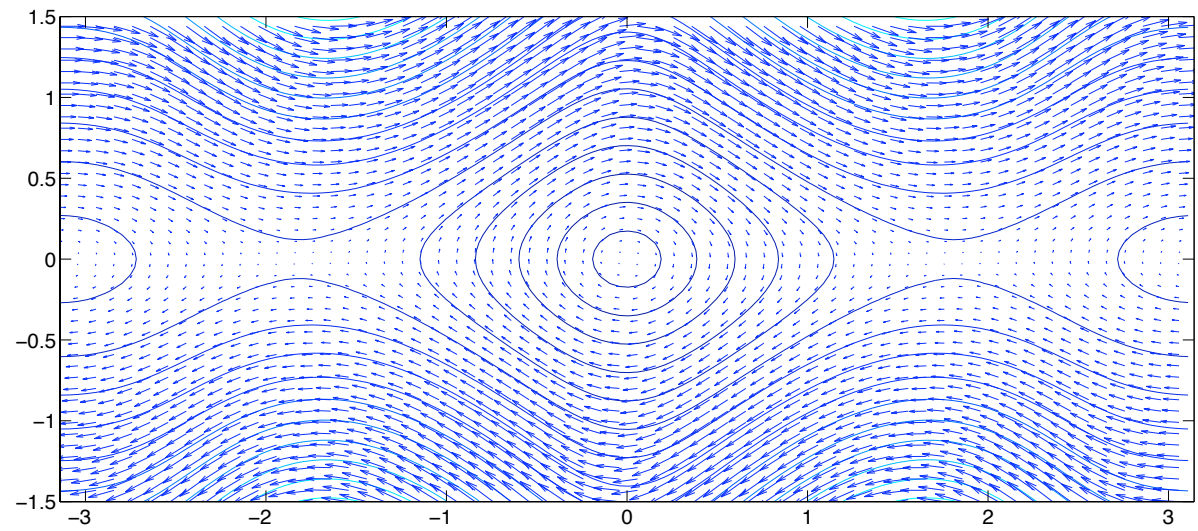

FIG. 4.6. One can observe two wells of potential on this phase portrait, connecting to Example 2. The reference configuration is still an ellipse characterized by the shape variable $\mathbf{c}_{\mathrm{ref}}=(1,0, \gamma, 0, \ldots, 0)$ as in Fig. 4.5 but the components of the rigidity matrix are here $\kappa_{3}=\kappa_{4}=0.2$ and $\kappa_{5}=\kappa_{6}=2$. The ellipse, the shape variable of which is $\mathbf{c}=(1,0,-\gamma, 0, \ldots, 0)$ is also a local minimizer of the potential energy of elasticity. This configuration, once turned into the form (4.17), corresponds to $\beta=\pi$. So the wells are centered at the points $(0,0)$ and $(\pi, 0)$.

The body experiences a net displacement along the $y$-axis. On Fig. 4.8 are displayed some screenshots of the motion of the body and on Fig. 4.9 is plotted the value of the $y$-coordinate of its center of mass with respect to the time. The shape changes are time periodic. On Fig. 4.10, are displayed the phase portraits of $a_{-1}$ and $b_{-2}$.

This locomotion can be termed passive because the motion is done at zero energy cost. The total energy of the system is conserved for all time. As shown on Fig. 4.11 and 4.12, there is, at any time, some exchanges of energy and impulse between the body and the fluid causing the net displacement. 


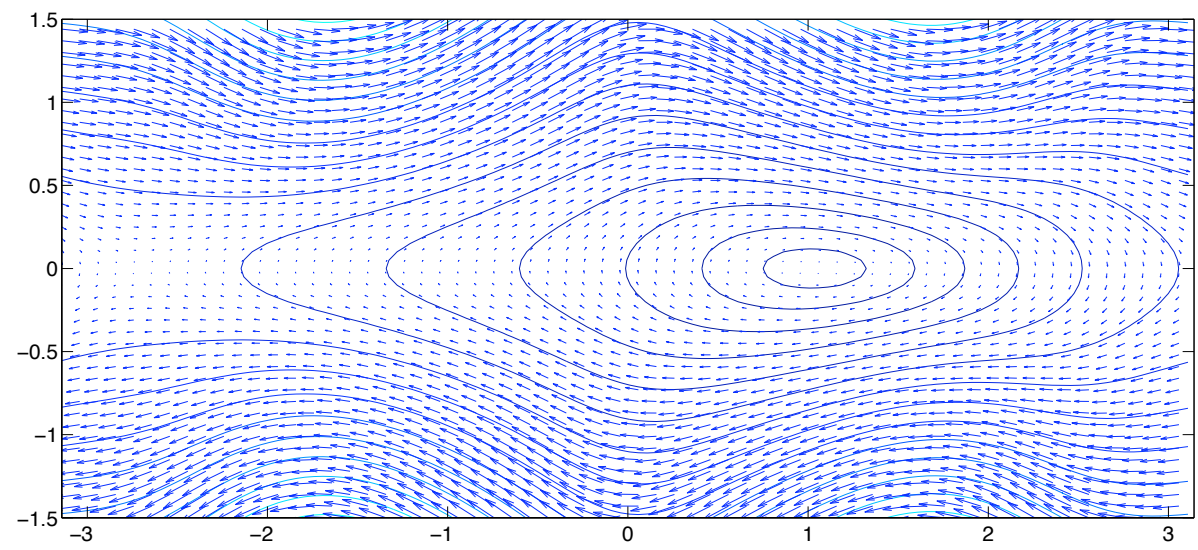

FIG. 4.7. To obtain this phase portrait for the second order ODE solved by $\beta$ (from Example 2), we have set $\gamma=0.5, \kappa_{j}=0.5(j=3,4,5,6)$ and we have chosen as reference configuration, that pictured on Subfigure 4.3(i) and whose the shape variable is $\mathbf{c}_{\mathrm{ref}}=(1,0,1 / 4,0,0, \sqrt{3} /(4 \sqrt{2}), 0 \ldots, 0)$. Once turned into the form (4.17), it corresponds to $\beta=\pi / 3$ and it can observed that the well of potential is precisely centered at the point $(\pi / 3,0)$. All the trapped solutions result in flapping motion while the others lead to a net displacement of the body along the $y$-axis.

Example 4: Non time periodic passive locomotion. Passive locomotion does not mean displacement along a straight line only. In the preceding data, we modify only the initial velocity $\dot{\mathbf{c}}_{0}$ (as detailed in the table below), exciting now the components $b_{-1}:=\Im\left(\mathbf{c}_{-1}\right)$ and $a_{-2}:=\Re\left(\mathbf{c}_{-2}\right)$ at the time $t=0$.

\begin{tabular}{|c|c|c|c|}
\hline $\mathrm{d}_{\text {free }}=5$ & $\varrho_{0}=1$ & $\alpha=0$ & $\varepsilon=*$ \\
\hline \multicolumn{4}{|c|}{$\mathbf{c}=\left(1,0, a_{-1}, b_{-1}, a_{-2}, b_{-2}, 0, \ldots, 0\right)$} \\
\hline \multicolumn{4}{|c|}{$\mathbb{R}=\operatorname{diag}(0,0,1,1,2,2,0, \ldots, 0)$} \\
\hline \multicolumn{4}{|c|}{$\mathbb{D}=0$ (no damping) } \\
\hline \multicolumn{4}{|c|}{$\mathbf{c}_{\mathrm{ref}}=(1,0,0.4899,0, \ldots, 0)$} \\
\hline \multicolumn{4}{|c|}{$\mathbf{c}_{0}=(1,0,0.4,0,0,0.2,0, \ldots, 0)$} \\
\hline \multicolumn{4}{|c|}{$\dot{\mathbf{c}}_{0}=(0,0,0.6,0.0450,0.1350,-0.6,0, \ldots}$, \\
\hline
\end{tabular}

As shown on Fig. 4.13, the body follows now a curved trajectory. Neither the strokes nor the components $a_{k}, b_{k}(k=-1,-2)$ are time periodic any more (see Fig. 4.14).

Example 5: Unlocking degrees of freedom, loss of time periodicity. We now unlock the variable $c_{1}:=a_{1}+i b_{2}$, adding two degrees of freedom to the body's shape changes (the total number of degrees of freedom for the deformations of the body is now 4). We compute the solution to the ODE (4.10) with the following data:

\begin{tabular}{|c|c|c|c|}
\hline $\mathrm{d}_{\text {free }}=7$ & $\varrho_{0}=1$ & $\alpha=0$ & $\varepsilon=*$ \\
\hline \multicolumn{4}{|c|}{$\mathbf{c}=\left(a_{1}, b_{1}, a_{-1}, b_{-1}, a_{-2}, b_{-2}, 0, \ldots, 0\right)$} \\
\hline \multicolumn{4}{|c|}{$\mathbb{R}=\operatorname{diag}(1,1,1,1,2,2,0, \ldots, 0)$} \\
\hline \multicolumn{4}{|c|}{$\mathbb{D}=0$ (no damping) } \\
\hline \multicolumn{4}{|c|}{$\mathbf{c}_{\mathrm{ref}}=(1,0,0.4899,0, \ldots, 0)$} \\
\hline \multicolumn{4}{|c|}{$\mathbf{c}_{0}=(1,0,0.4,0,0,0.2,0, \ldots, 0)$} \\
\hline \multicolumn{4}{|c|}{$\dot{\mathbf{c}}_{0}=(0.2121,0,0.6152,0,0,-0.0848,0, \ldots$} \\
\hline
\end{tabular}

With the first three components $c_{k}(k=1,-1,-2)$ of the shape-variable unlocked, it is more complicated to find a rigidity matrix $\mathbb{R}$ and initial data $\mathbf{c}_{0}$ and $\dot{\mathbf{c}}_{0}$ that produce locomotion. Although 


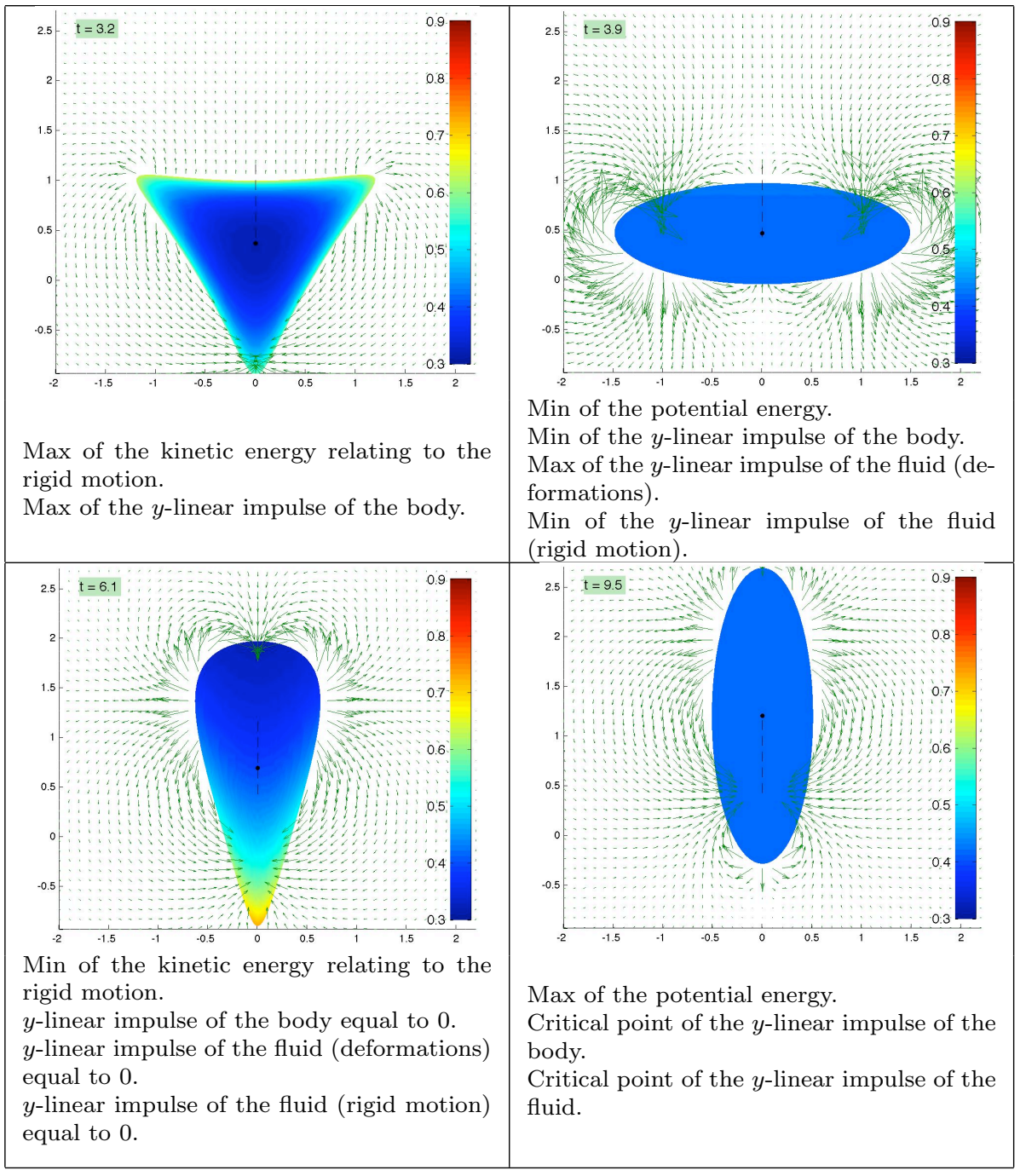

FIG. 4.8. Screenshots of the swimmer of Example 3 at the times $t=3.17,3.95,6.07$ and 9.50 corresponding to instants which are relevant to the energy and linear impulse distribution (see Fig. 4.10 and Fig. 4.11). The black spot is the position of the center of mass and the colors correspond to the density of the body. The dashed line is the trajectory of the center of mass. The Eulerian velocity field of the fluid is represented by the green arrows.

the strokes seem to be periodic on Fig. 4.15, they are actually not, as it can be observed on Fig. 4.16. In this example, the body experiences a passive locomotion with net displacement along the $y$-axis. The position of its center of mass with respect to the time is plotted on Fig. 4.17.

Example 6: Hydrodynamical coupling. The number of degrees of freedom relating to the shape changes is the same as in the preceding example but the unlocked components are now all of the $c_{k}$ for $k=-1,-2,-3$. The initial data and the rigidity matrix are the same as in Example 3. It means in particular that the component $c_{-3}:=a_{-3}+i b_{-3}$ is equal to zero and not excited at 


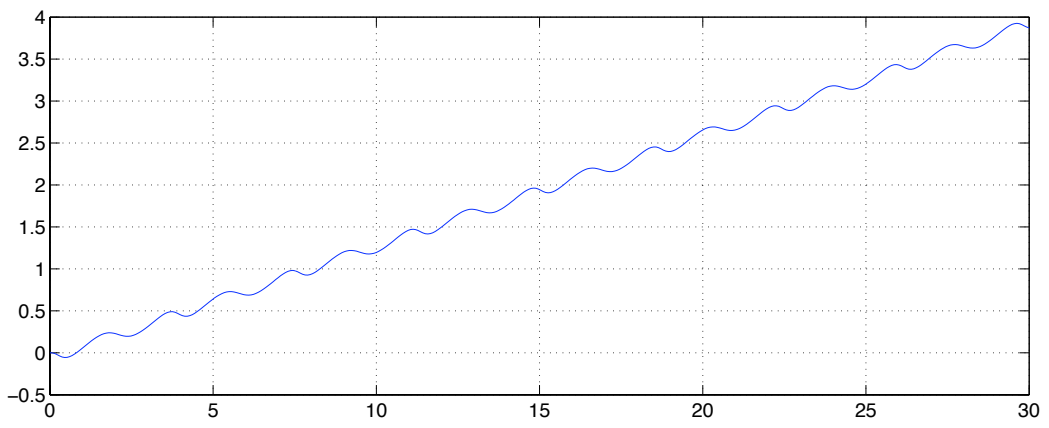

FIG. 4.9. y-coordinate of the center of mass of the swimmer (Example 3) with respect to time. In this case, the strokes causing the displacement are time periodic.

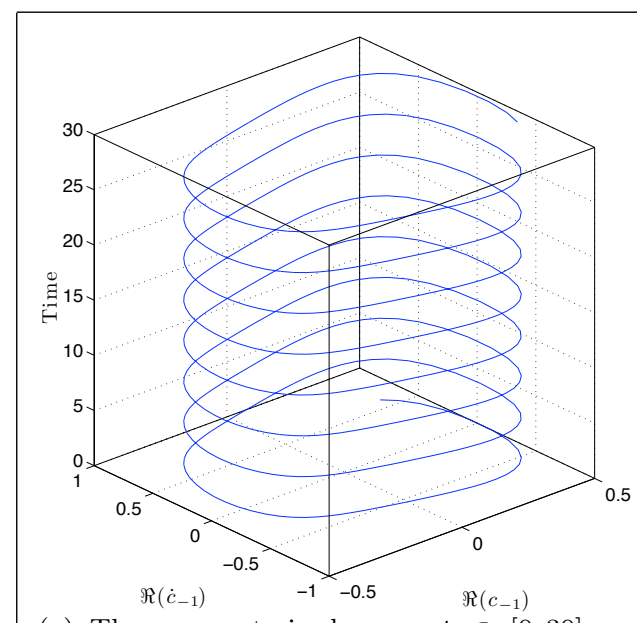

(a) The parameterized curve $t \in[0,30] \mapsto$ $\left(\Re\left(\mathbf{c}_{-1}(t)\right), \Re\left(\dot{\mathbf{c}}_{-1}(t)\right), t\right) \in \mathbf{R}^{3}$

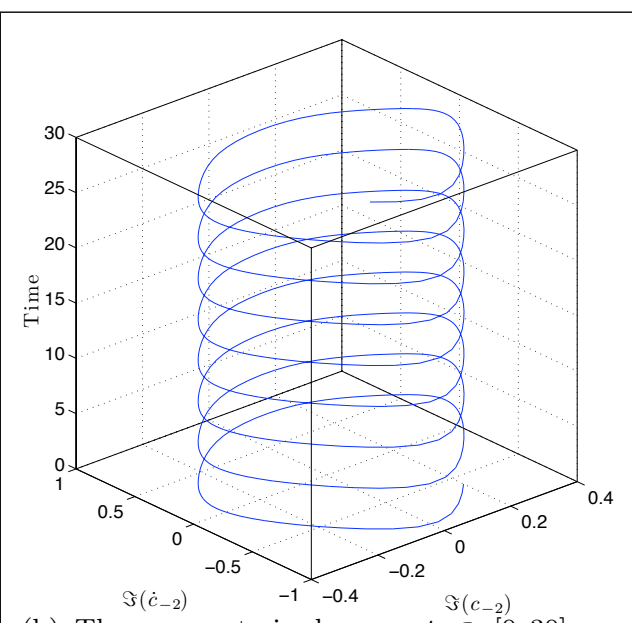

(b) The parameterized curve $t \in[0,30] \mapsto$ $\left(\Im\left(\mathbf{c}_{-2}(t)\right), \Im\left(\dot{\mathbf{c}}_{-2}(t)\right), t\right) \in \mathbf{R}^{3}$

FIG. 4.10. Phase portraits of the components $a_{-1}:=\Re\left(\mathbf{c}_{-1}\right)$ and $b_{-2}:=\Im\left(\mathbf{c}_{-2}\right)$ for the swimmer of Example 3. Both components are time periodic. The resulting shape changes are time periodic strokes as well, causing a net displacement along the $y$-axis.

the initial time.

\begin{tabular}{|l|c|c|c|}
\hline \multicolumn{1}{|c|}{$\mathrm{d}_{\text {free }}=7$} & $\varrho_{0}=1$ & $\alpha=0$ & $\varepsilon=*$ \\
\hline $\mathbf{c}=\left(1,0, a_{-1}, b_{-1}, a_{-2}, b_{-2}, a_{-3}, b_{-3}, 0, \ldots, 0\right)$ \\
\hline $\mathbb{R}=\operatorname{diag}(0,0,1,1,2,2,30,30,0, \ldots, 0)$ \\
\hline $\mathbb{D}=0$ (no damping) \\
\hline $\mathbf{c}_{\text {ref }}=(0,0,0.4899,0, \ldots, 0)$ \\
\hline $\mathbf{c}_{0}=(1,0,0.4,0,0,0.2,0, \ldots, 0)$ \\
\hline$\dot{\mathbf{c}}_{0}=(0,0,0.6,0,0,-0.6,0, \ldots, 0)$ \\
\hline
\end{tabular}

Once more, we obtain an example of passive locomotion. Some screenshots of the swimming body are displayed on Fig. 4.18. Since the rigidity matrix $\mathbb{R}$ is diagonal, the elastic modes corresponding to the component $c_{-3}$ would not have been excited in the absence of fluid (see Fig. 4.19). On 


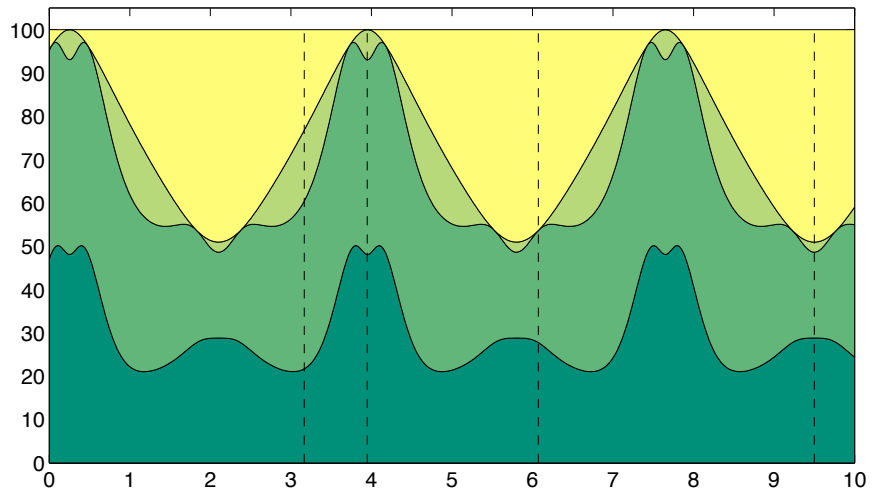

FIG. 4.11. Distribution of energy (in percents) with respect to the time for the swimmer of Example 3. The overall energy of the fluid-swimmer system can be decomposed into the kinetic energy and the potential energy of elasticity. The kinetic energy can be further decomposed into the kinetic energy of the fluid and the kinetic energy of the body. Eventually, the kinetic energy of the body is the sum of the kinetic energy caused by the shape changes and the the kinetic energy resulting from the rigid displacement. In this graphic, the amount of energy of each type is proportional to the area. The vertical dashed straight lines highlight 4 instants of interest regarding the energy and momentum distributions. They are detailed on Fig. 4.8.

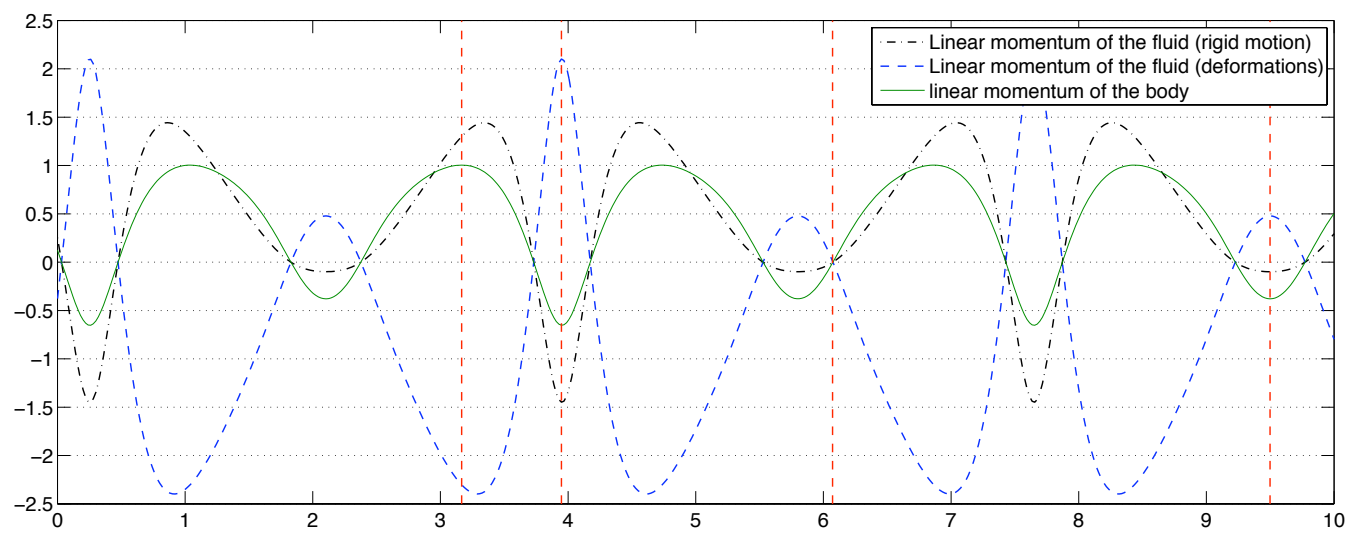

FIG. 4.12. Since the displacement of the swimmer of Example 3 is along the $y$-axis only and the shape changes are symmetric with respect to this axis, the flow is also symmetric and the $x$-component of the linear impulse of the fluid is equal to zero for all times. The y-linear impulse of the fluid-swimmer system can be decomposed into the sum of the linear impulse of the fluid (caused either by the shape changes or the rigid motion of the body) and the impulse of the body. The vertical dashed lines highlight the same instants as on Fig. 4.11, representing some interest regarding the exchange of impulse.

Fig. 4.20, one can observe the distribution of energy during a little more than 2 strokes. The body swims along the $y$-axis. The position of its center of mass with respect to the time is plotted on Fig. 4.21 .

Example 7: What happens with a even softer body?. We unlock now all of the 8 complex components of the shape variable. It means that 14 degrees of freedom are allowed in the shape changes. Locomotion is quite hard to achieve with such a soft body. The rigidity matrix $\mathbb{R}$ 


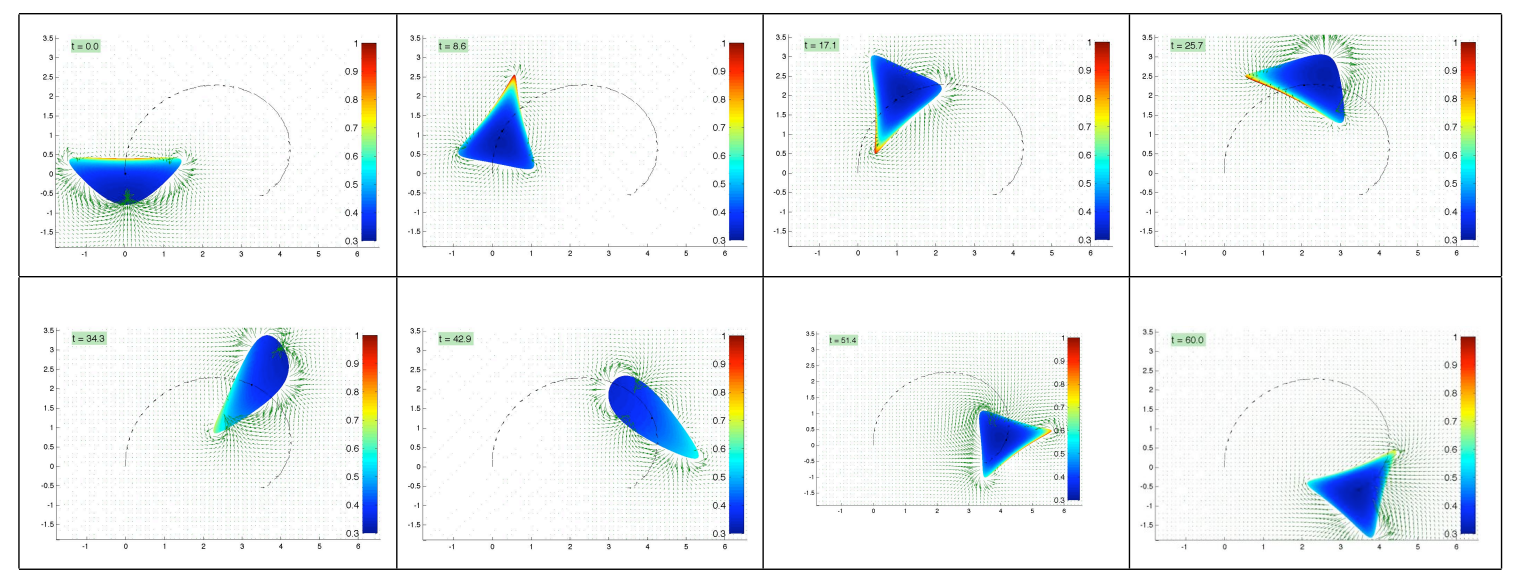

FIG. 4.13. Screenshots of the motion of the swimmer (Example 4) over the time interval [0,60]. The dashed black line shows the trajectory of the center of mass. The colors give the value of the internal density (the animation can be found on the web page of which the location is given at the beginning of this subsection).

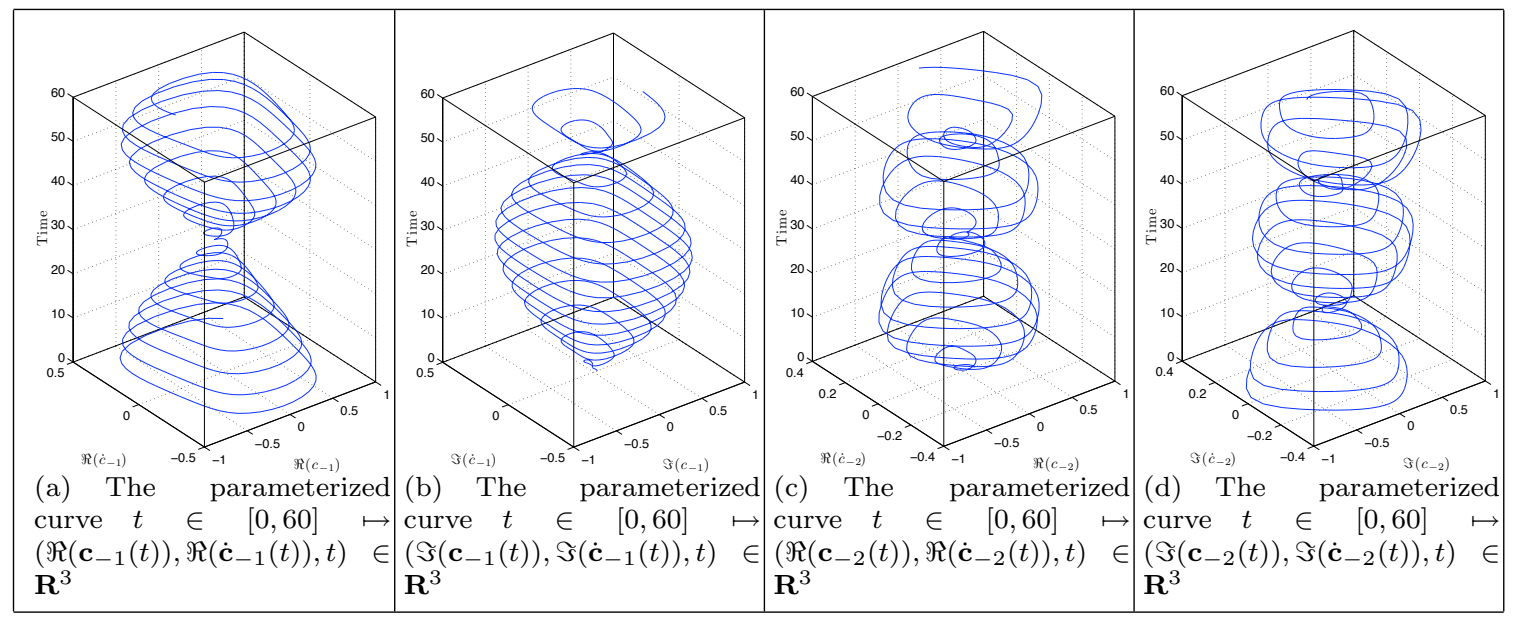

FIG. 4.14. Phase portraits of the variables $a_{-1}:=\Re\left(\mathbf{c}_{-1}\right), b_{-1}:=\Im\left(\mathbf{c}_{-1}\right), a_{-2}:=\Re\left(\mathbf{c}_{-2}\right)$ and $b_{-2}:=\Im\left(\mathbf{c}_{-2}\right)$ for the swimmer of Example 4. None of them seem to be time periodic.

is set in such a way that the modes $c_{k}$ for $k=1$ and $k \leq-3$ have a high stiffness coefficient (i.e. a large amount of energy is necessary to deform the body along these modes). We have to introduce some dissipation effects and to use the potential energy $W_{2}$ to prevent infinite concentration of material (i.e. infinite density inside the body). 


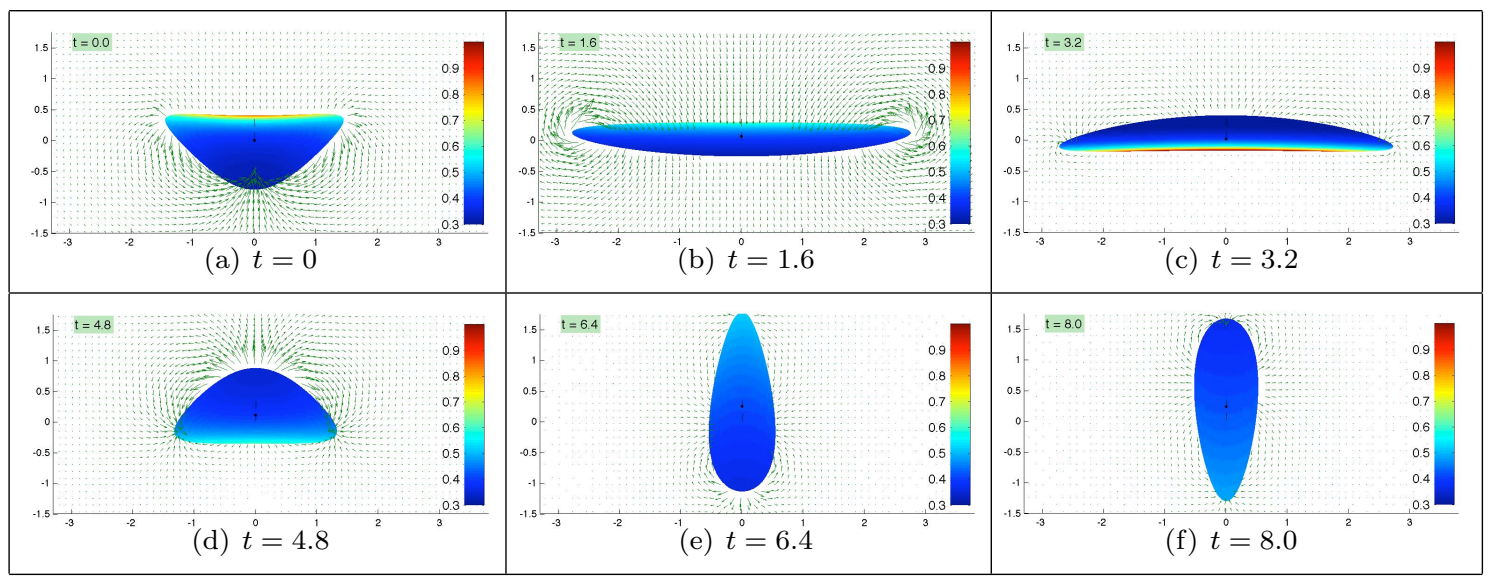

FIG. 4.15. Screenshots of the motion of the body described in Example 5 over the time interval [0,8]. The colors give the value of the internal density. The shape changes are not time periodic in this example (the animation can be found on the web page of which the location is given at the beginning of this subsection).
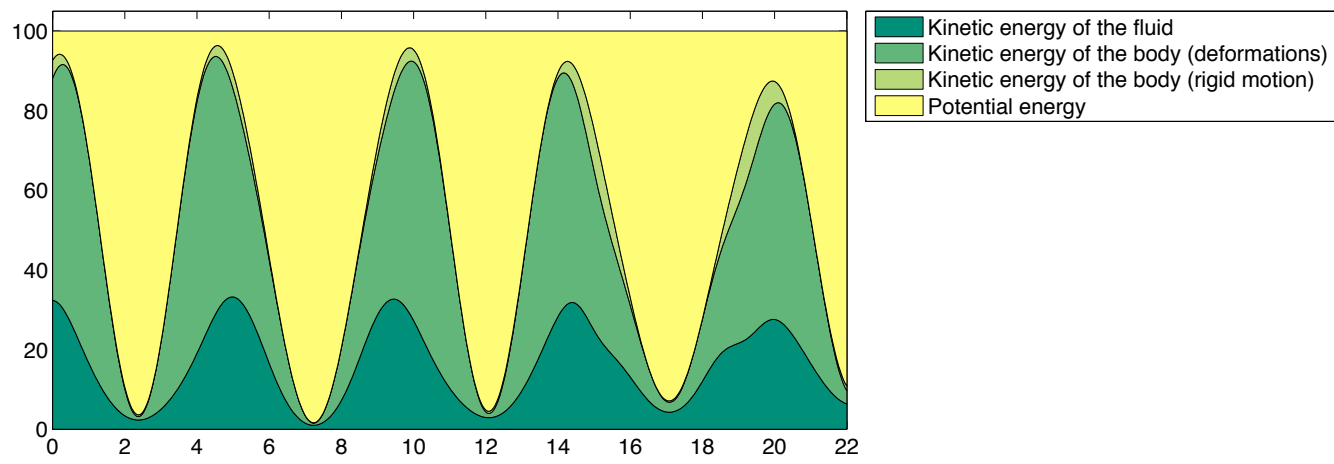

FIG. 4.16. Time evolution of the distribution of energy (in percent) for the swimmer of Example 5. Each peak corresponds to a stroke. One can observe that the peaks are different, which means that the strokes are not time periodic. Compared to Example 3, the percentage of the body's kinetic energy (relating to the rigid displacement) is smaller, making this way of swimming less efficient.

\begin{tabular}{|c|c|c|c|}
\hline $\mathrm{d}_{\text {free }}=17$ & $\varrho_{0}=1$ & $\alpha=0.5$ & $\varepsilon=0.03$ \\
\hline \multicolumn{4}{|c|}{$\mathbf{c}=\left(a_{1}, b_{1}, a_{-1}, b_{-1}, a_{-2}, b_{-2}, a_{-3}, b_{-3}, a_{-4}, b_{-4}, a_{-5}, b_{-5}, a_{-6}, b_{-6}, a_{-7}, b_{-7}\right)$} \\
\hline \multicolumn{4}{|c|}{$\mathbb{R}=\operatorname{diag}(100,100,1,1,1,1,100,100,100,100,100,100,100,100,100,100)$} \\
\hline \multicolumn{4}{|c|}{$\mathbb{D}=\operatorname{diag}(0.1,0.1,0.1,0.1,0.2,0.2,0.3,0.3,0.4,0.4,0.5,0.5,0.6,0.6,0.7,0.7)$} \\
\hline \multicolumn{4}{|c|}{$\mathbf{c}_{\mathrm{ref}}=(0.8908,0,0.3984,0, \ldots, 0)$} \\
\hline \multicolumn{4}{|c|}{$\mathbf{c}_{0}=(1,0,0.5,0,0,0.24,0, \ldots, 0)$} \\
\hline \multicolumn{4}{|c|}{$\dot{\mathbf{c}}_{0}=(0.2026,0,0.4987,0,0,-0.0973,0, \ldots, 0)$} \\
\hline
\end{tabular}

Because of the damping effects, only three strokes are possible before the total amount of energy becomes insufficient for the body to swim. Screenshots are displayed on Fig. 4.22 and the position of the center of mass with respect to time is plotted on Fig. 4.23. Although only three components out of the 16 of the shape variable are excited at the initial time, due to the hydrodynamical coupling 


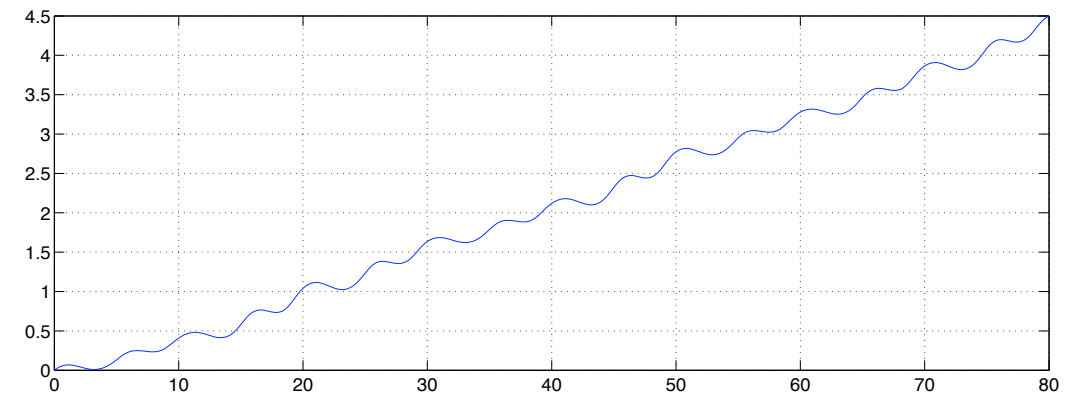

FIG. 4.17. $y$-coordinate of the center of mass of the body (Example 5) with respect to time.

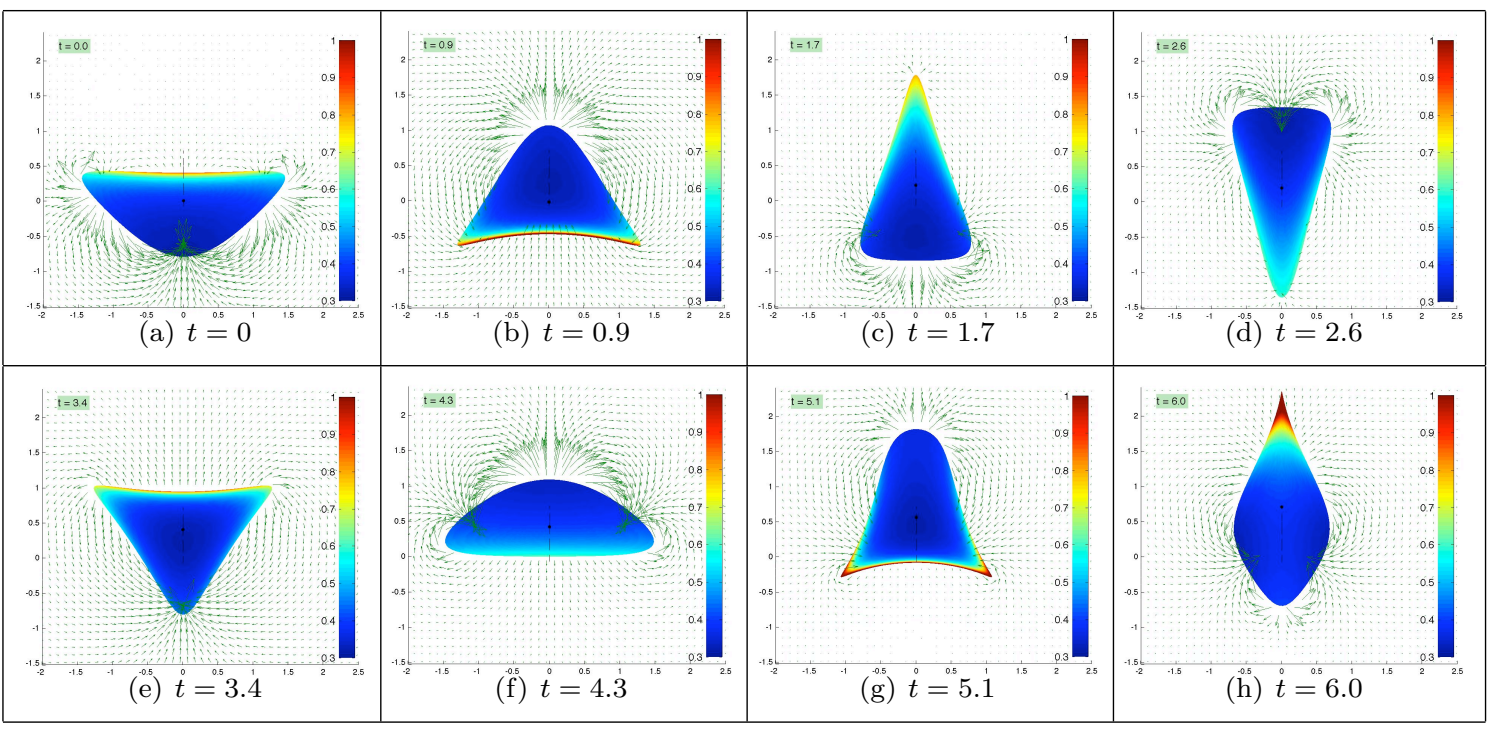

FIG. 4.18. Screenshots of the motion of the swimmer of Example 6 over the time interval [0,6]. The strokes are clearly not periodic (the animation can be found on the web page of which the location is given at the beginning of this subsection).

and the potential energy $W_{2}$, all of the modes get excited, as it can be observed on Fig. 4.24 and 4.25 .

5. Self-propelled Locomotion. We consider now that the fluid-swimmer system is at rest at the time $t=0$ (the energy of the overall system is equal to 0 ) and that the body is able to modify its shape by means of some internal forces (for instance, the forces could be resulting from the work of the muscles of a fish). This new feature can easily be incorporated to our model.

5.1. Equation of motion. Equation (4.13) has to be turned into:

$$
\left(\frac{d}{d t} \frac{\partial \mathcal{L}}{\partial \dot{\mathbf{c}}}(\mathbf{c})-\frac{\partial \mathcal{L}}{\partial \mathbf{c}}(\mathbf{c})\right)-\sum_{j=1}^{N^{\prime}} \lambda_{j}(\mathbf{c}, \dot{\mathbf{c}}) G_{j}(\mathbf{c})=\mathbf{F},
$$




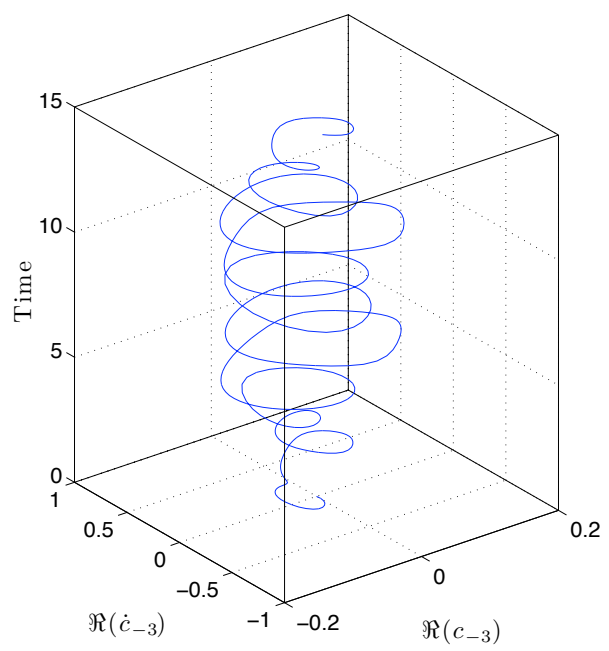

FIG. 4.19. Phase portrait of the component $c_{-3}$ (parameterized curve $t \in[0,15] \mapsto\left(\Re\left(\mathbf{c}_{-3}(t)\right), \Re\left(\dot{\mathbf{c}}_{-3}(t)\right), t\right) \in$ $\mathbf{R}^{3}$ ) connecting to Example 6. Although not excited at the initial time, this mode gets excited because of the hydrodynamical coupling.
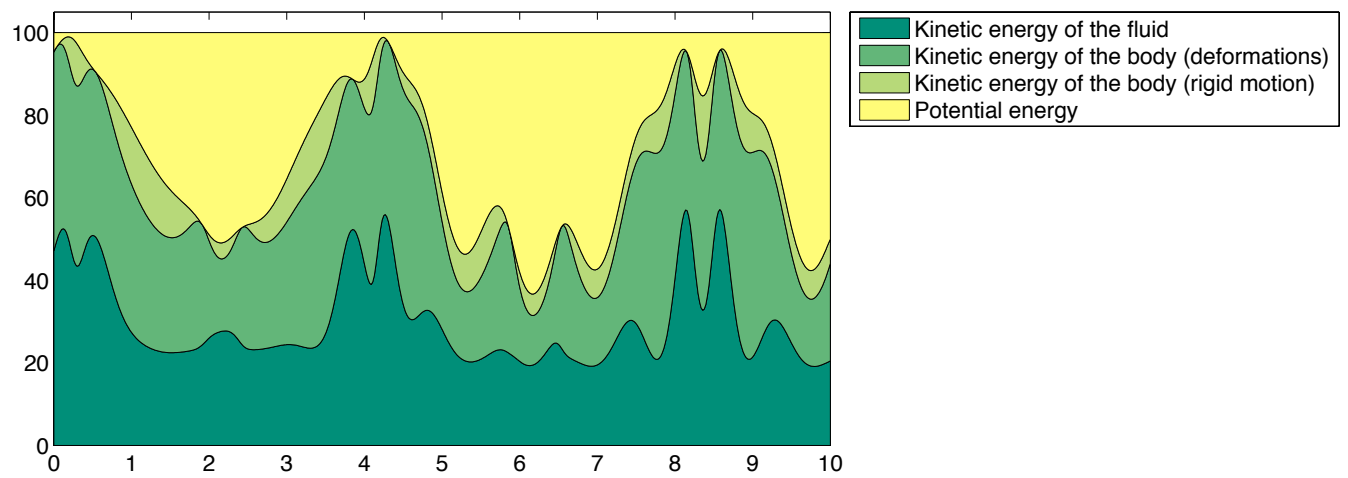

FIG. 4.20. Time evolution of the distribution of energy (in percentage) for the swimmer of Example 6. The strokes can still be identified; they correspond to the peaks. They are clearly not time periodic.

where the function $\mathbf{F}: t \in \mathbf{R}_{+} \rightarrow \mathbf{C}^{N+1}$ stands for the internal forces (usually called generalized forces in Lagrangian Mechanics) exerted by the swimming body. In the normal form (4.10), we have to modify the expression $(4.10 \mathrm{~b})$ of $\Lambda(\mathbf{c}, \dot{\mathbf{c}})$ as follows:

$$
\Lambda(\mathbf{c}, \dot{\mathbf{c}}):=-\mathbb{K}(\mathbf{c})^{-1}\langle\Gamma(\mathbf{c}), \dot{\mathbf{c}}, \dot{\mathbf{c}}\rangle-\mathbb{K}(\mathbf{c})^{-1} \frac{\partial W}{\partial \mathbf{c}}(\mathbf{c})+\mathbb{K}(\mathbf{c})^{-1} \mathbf{F} .
$$

We claim:

TheOREm 5.1. For any integer $N \geq 1$, for any $\mu>0$, for any initial data $\left(\mathbf{c}_{0}, \dot{\mathbf{c}}_{0}\right) \in \mathcal{A}_{N}^{\bullet}(\mu) \times \mathcal{S}_{N}$ satisfying the compatibility conditions $\left(G_{j}\left(\mathbf{c}_{0}\right), \dot{\mathbf{c}}_{0}\right)_{\ell^{2}(\mathbf{C})}=0\left(j=1, \ldots, N^{\prime}\right)$ and for any Lipschitz continuous function $\mathbf{F}: t \in \mathbf{R}_{+} \mapsto \mathbf{C}^{N+1}$, there exists a unique $\mathcal{C}^{2}$ solution $t \in[0,+\infty[\mapsto \mathbf{c}(t) \in$ $\mathcal{A}_{N}(\mu)$ to ODE (4.10a-5.2) satisfying $(\mathbf{c}(0), \dot{\mathbf{c}}(0))=\left(\mathbf{c}_{0}, \dot{\mathbf{c}}_{0}\right)$. Besides, if $\varepsilon>0$ and $\alpha \geq 1 / 2$ in the 


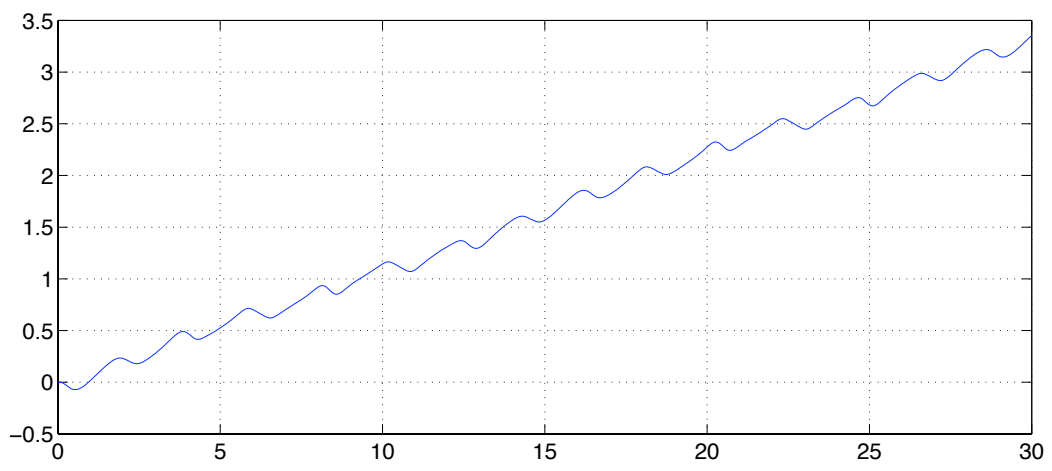

FIG. 4.21. $y$-coordinates of the center of mass of the body (Example 6) with respect to the time.

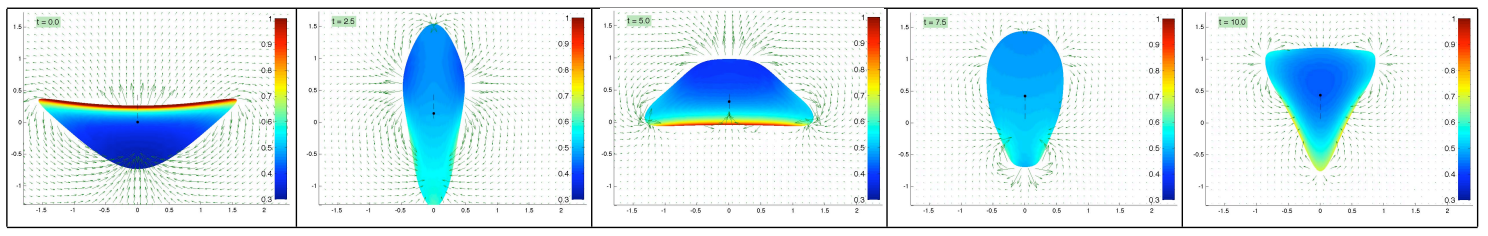

Fig. 4.22. Screenshots of the motion of the swimmer (Example 7) at the times $t=0,2.5,5,7.5$ and 10. The colors correspond to the density of the body (the animation can be found on the web page of which the location is given at the beginning of this subsection).

definition of $W_{2}$ (see Proposition 4.2) the solution is allowable (in the sense of Definition 2.2) and analytic if the functions $V, G_{j}$ and $\mathbf{F}$ are.

Proof. As in the proof of Theorem 4.5, the Cauchy-Lipschitz Theorem applies and guarantees that there exists a unique $\mathcal{C}^{2}$ (or analytic if $V, G_{j}$ and $\mathbf{F}$ are) solution over a time interval $[0, T$ ) for some $T>0$. The energy estimate (4.14) becomes, for all $0<t<T$ :

$$
\frac{d}{d t} E=\left(\frac{d}{d t} \frac{\partial \mathcal{L}}{\partial \dot{\mathbf{c}}}(\mathbf{c})-\frac{\partial \mathcal{L}}{\partial \mathbf{c}}(\mathbf{c}), \dot{\mathbf{c}}\right)_{\ell^{2}(\mathbf{C})}=(\mathbf{F}, \dot{\mathbf{c}})_{\ell^{2}(\mathbf{C})}
$$

Integrating over $[0, t]$ for any $0<t<T$ and invoking the inequality (4.9) we get:

$$
\frac{\nu_{N}}{2}\|\dot{\mathbf{c}}\|_{\ell^{2}(\mathbf{C})}^{2} \leq E(t) \leq E(0)+\int_{0}^{t}\|\mathbf{F}(s)\|_{\ell^{2}(\mathbf{C})}\|\dot{\mathbf{c}}(s)\|_{\ell^{2}(\mathbf{C})} \mathrm{d} s .
$$

Setting then:

$$
\Upsilon(t):=\int_{0}^{t}\|\mathbf{F}(s)\|_{\ell^{2}(\mathbf{C})}\|\dot{\mathbf{c}}(s)\|_{\ell^{2}(\mathbf{C})} \mathrm{d} s,
$$

we obtain after some basic algebra that, for all $0<t<T$ :

$$
\frac{\Upsilon^{\prime}(t)}{\sqrt{\Upsilon(t)+E(0)}} \leq \frac{\|\mathbf{F}(t)\|_{\ell^{2}(\mathbf{C})}}{\sqrt{\nu_{N} / 2}} .
$$




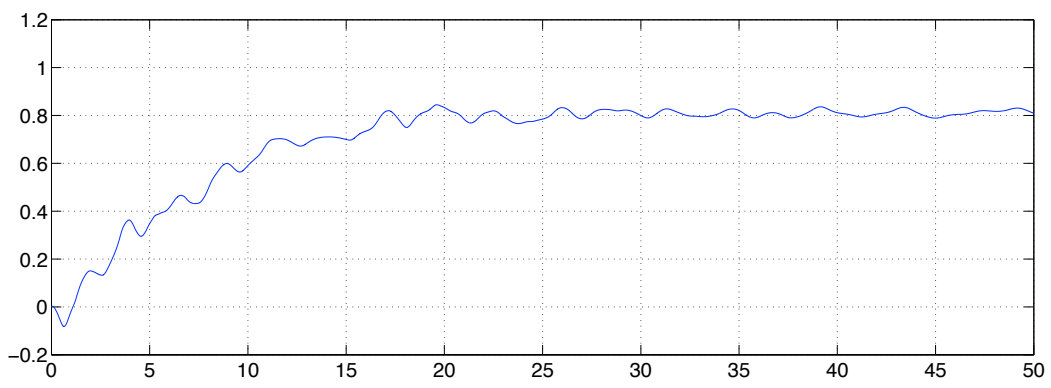

FIG. 4.23. y-coordinate of the center of mass of the swimmer (Example 7) with respect to the time.

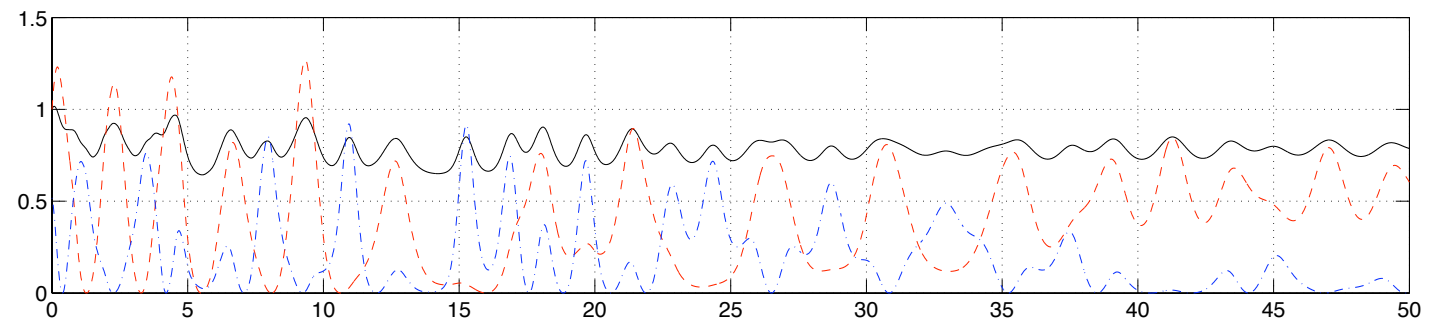

FIG. 4.24. The black, red and blue curves correspond respectively to the components $\left|c_{1}(t)\right|^{2}, 4\left|c_{-1}(t)\right|^{2}$ and $9\left|c_{-2}(t)\right|^{2}$ of the shape variable (Example 7).

Integrating this inequality with respect to time, we get the estimate:

$$
\Upsilon(t) \leq E(0)+\left[\sqrt{E(0)}+\frac{1}{\sqrt{2 \nu_{N}}} \int_{0}^{t}\|\mathbf{F}(s)\|_{\ell^{2}(\mathbf{C})} \mathrm{d} s\right]^{2} .
$$

Substituting this result into (5.4), we obtain the Gronwall-type inequality:

$$
\frac{1}{2}\|\dot{\mathbf{c}}\|_{\ell^{2}(\mathbf{C})}^{2} \leq \frac{2}{\nu_{N}} E(0)+\frac{1}{\nu_{N}}\left[\sqrt{E(0)}+\frac{1}{\sqrt{2 \nu_{N}}} \int_{0}^{t}\|\mathbf{F}(s)\|_{\ell^{2}(\mathbf{C})} \mathrm{d} s\right]^{2}
$$

meaning that $\dot{\mathbf{c}}$ remains bounded for all $t \in[0, T)$. We next conclude as in the proof of Theorem 4.5. 口

5.2. Numerical simulations: How to make a soft ball swim?. In this example, the shape of the swimmer is a ball at the initial time. This shape is also its reference configuration (minimizing the potential energy of elasticity). Under the action of some internal forces, the ball undergoes shape changes that produce a net displacement along the $y$-axis. As usual, the data are displayed in the table below. In the last row, we give the expression of the internal forces as a vector of 16 time depending components. Notice that the fluid-body system is at rest at the time $t=0$. 


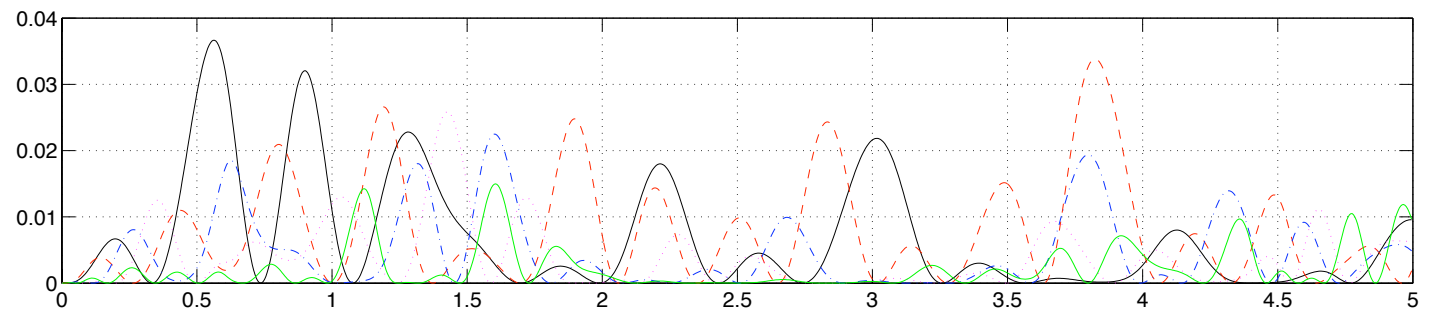

FIG. 4.25. The black, red, blue, magenta and green curves correspond respectively to the components $16\left|c_{-3}(t)\right|^{2}$, $25\left|c_{-4}(t)\right|^{2}, 36\left|c_{-5}(t)\right|^{2}, 49\left|c_{-6}(t)\right|^{2}$ and $64\left|c_{-7}(t)\right|^{2}$ of the shape variable (Example 7). These modes are not excited at the time $t=0$. They get excited through $W_{2}$ and the influence of the fluid which couples all of the modes.

\begin{tabular}{|c|c|c|c|}
\hline $\mathrm{d}_{\text {free }}=17$ & $\varrho_{0}=1$ & $\alpha=0.5$ & $\varepsilon=0.03$ \\
\hline \multicolumn{4}{|c|}{$\mathbf{c}=\left(a_{1}, b_{1}, a_{-1}, b_{-1}, a_{-2}, b_{-2}, a_{-3}, b_{-3}, a_{-4}, b_{-4}, a_{-5}, b_{-5}, a_{-6}, b_{-6}, a_{-7}, b_{-7}\right)$} \\
\hline \multicolumn{4}{|c|}{$\mathbb{R}=\operatorname{diag}(10,10,1,1,1,1,3,3,4,4,5,5,6,6,7,7)$} \\
\hline \multicolumn{4}{|c|}{$\mathbb{D}=\operatorname{diag}(0.2,0.2,0.2,0.2,0.4,0.4,0.6,0.6,0.8,0.8,1,1,1.2,1.2,1.4,1.4)$} \\
\hline \multicolumn{4}{|l|}{$\mathbf{c}_{\mathrm{ref}}=(1,0, \ldots, 0)$} \\
\hline \multicolumn{4}{|l|}{$\mathbf{c}_{0}=(1,0, \ldots, 0)$} \\
\hline \multicolumn{4}{|l|}{$\dot{\mathbf{c}}_{0}=(0, \ldots, 0)$} \\
\hline $\mathbf{F}(t)=[2(4 \operatorname{arct}$ & $\mathrm{s}(t / 3$ & 0 , & \\
\hline
\end{tabular}

On Fig. 5.1 are displayed some screenshots of the body during its swimming. The position of its center of mass with respect to time is plotted on Fig. 5.2 and on Fig. 5.3 is shown the distribution of energy.

6. Conclusion. In this paper, we have presented a model to study the dynamic interaction between a deformable body (which can be made of a hyperelastic material) and a perfect fluid. This model has allowed us to find out some interesting properties, the most noteworthy being that passive locomotion with zero initial velocity, is possible even when the body is not even elastic. In this case, the swimmer is completely passive and its deformations are caused by the hydrodynamical forces exerted by the fluid on its boundary. The fluid-swimmer system is naturally oscillating and the body, driven by the fluid, undergoes time periodic shape changes resulting in locomotion. This result casts a new light on some studies of swimming efficiency, such as [14] or [25], in which the cost function involves the forces (or the torques) exerted by the swimmer. Indeed, the locomotion strategy presented in Example 1 is optimal in terms of both the energy expanded over a stroke and the internal force exerted by the body (the cost being zero in both cases). This result should not be confused with D'Alambert's paradox but rather be understood as a generalization of this quite counterintuitive phenomenon to deformable immersed bodies.

Appendix A. Sequences of complex numbers. We recall that $\mathcal{S}$ is the Banach space consisting of the complex sequences $\mathbf{c}:=\left(c_{k}\right)_{k \leq 1}, c_{k}=a_{k}+i b_{k}, a_{k}, b_{k} \in \mathbf{R}$ such that $c_{0}=0$ and $\|\mathbf{c}\|_{\mathcal{S}}:=\sum_{k \leq 1}|k|\left(\left|a_{k}\right|+\left|b_{k}\right|\right)<+\infty$. The indexation of the elements $c_{k}$ by integers $k$ ranging from 1 to $-\infty$ may seem confusing but fits with the definition (2.2) of the conformal mapping $\phi(\mathbf{c})$.

For any $N \in \mathbf{N}$, the $(N+1)$-dimensional subspace of $\mathcal{S}$ for which $c_{k}=0$ if $k<-N$ is denoted by $\mathcal{S}_{N}$. It will be identified with $\mathbf{C}^{N+1}$ or $\mathbf{R}^{2(N+1)}$ and its elements denoted respectively by $\mathbf{c}:=\left(c_{1}, c_{-1}, \ldots, c_{-N}\right) \in \mathbf{C}^{N+}$ or $\mathbf{c}:=\left(a_{1}, b_{1}, a_{-1}, b_{-1}, \ldots, a_{-N}, b_{-N}\right) \in \mathbf{R}^{2(N+1)}$ (we drop the element $c_{0}:=a_{0}+i b_{0}$, which is always equal to 0$)$. 


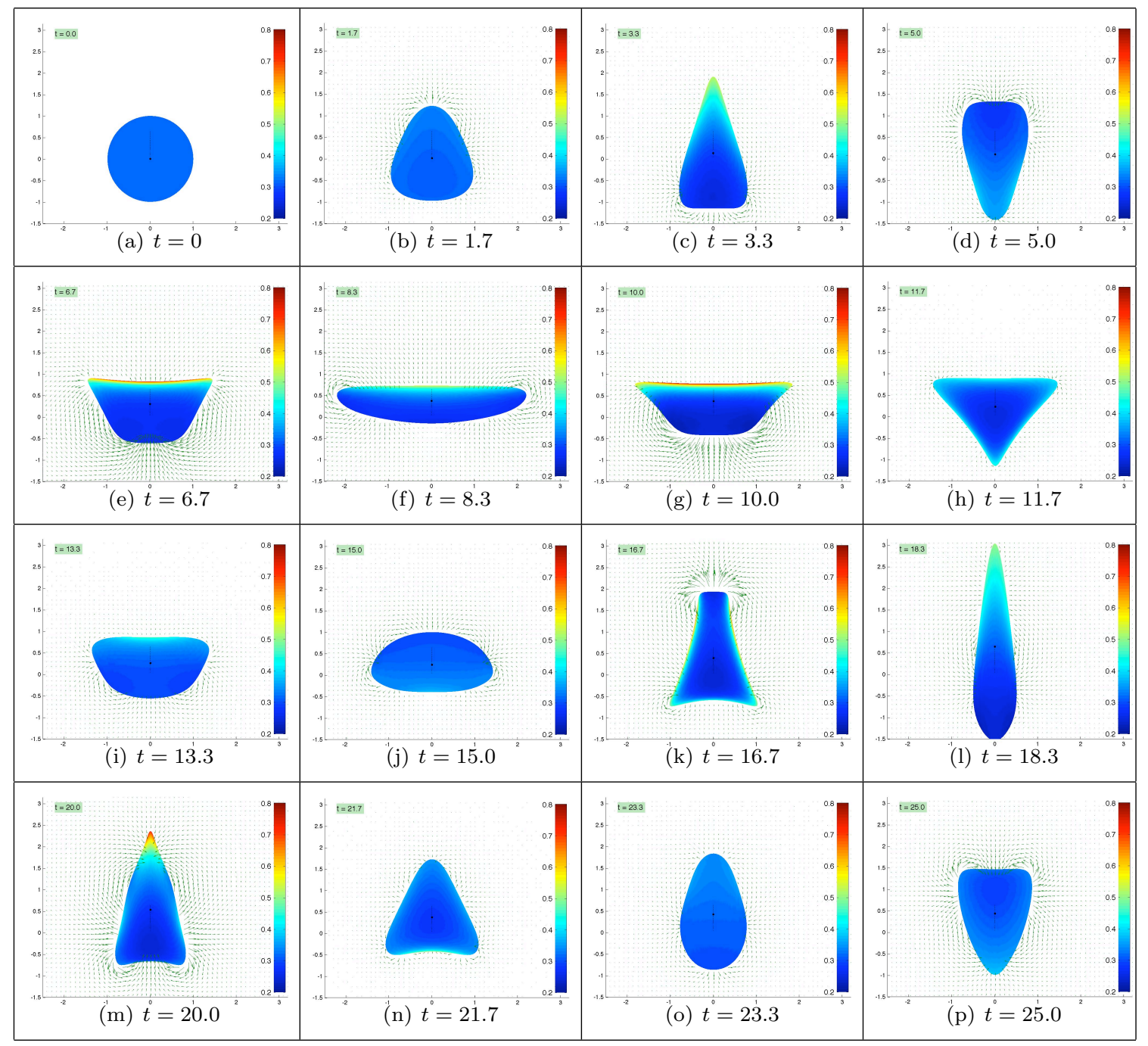

FIG. 5.1. Screenshots of the motion of the body over the time interval [0,25] (self-propeled swimmer). The colors give the value of the internal density (the animation can be found on the web page of which the location is given at the beginning of this subsection).

Appendix B. Multilinear, polynomial and analytic functions. Let $E_{1}, \ldots, E_{k}(k \geq 1)$ be Banach spaces. The set consisting of all the continuous, $k$-linear mappings from $E:=E_{1} \times \ldots \times E_{k}$ into $F$ is denoted $\mathcal{L}_{k}(E, F)$ (we will drop the subscript $k$ when $k=1$ ). It is a Banach space whose the norm is classically defined by:

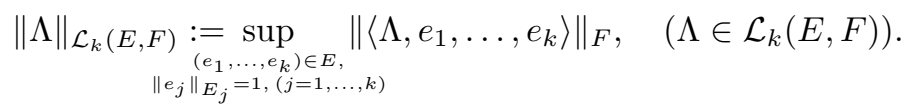

When $F=\mathbf{R}$, we are dealing with multilinear continuous forms and we denote merely $\mathcal{L}_{k}(E):=$ 


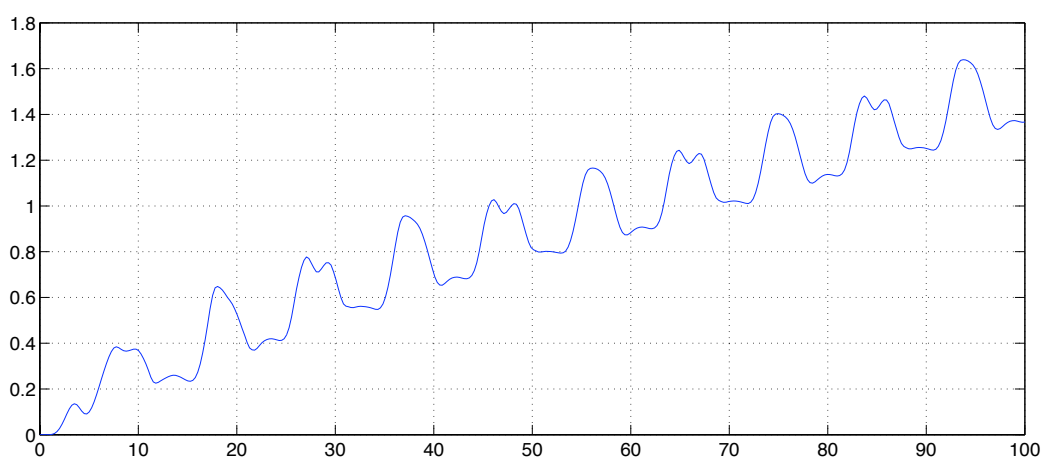

FIG. 5.2. $y$-coordinate of the center of mass of the self-propeled swimmer with respect to the time.

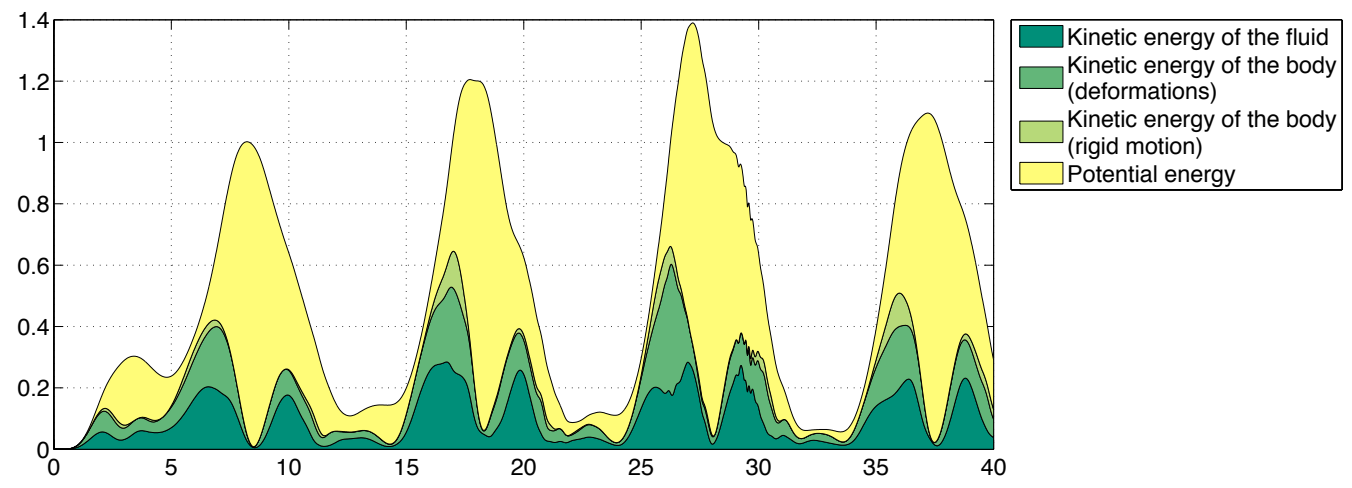

FIG. 5.3. Time evolution of the distribution of energy for the self-propeled swimmer. Due to the action of the internal forces, the overall energy of the fluid-body system is not constant any more. The system is at rest at the time $t=0$.

\section{$\mathcal{L}_{k}(E, F)$.}

We call polynomial function from a Banach space $E$ (or from only a subset of this space) into a Banach space $F$ any function $P$ such that there exists an integer $p \in \mathbf{N}$ (the degree of the polynomial $), A_{0} \in F$ and $p$ mappings $A_{k} \in \mathcal{L}_{k}\left(E^{k}, F\right)(k=1, \ldots, p)$ such that:

$$
P(e):=A_{0}+\sum_{k=1}^{p}\left\langle A_{k}, e, \ldots, e\right\rangle, \quad \forall e \in E .
$$

We denote $\mathcal{P}(E, F)$ the set of all the polynomial functions from $E$ into $F$. Observe that in particular $\mathcal{L}(E, F) \subset \mathcal{P}(E, F)$. We easily prove that if $E_{1}, E_{2}$ and $E_{3}$ are three Banach spaces and $P_{1} \in$ $\mathcal{P}\left(E_{1}, E_{2}\right), P_{2} \in \mathcal{P}\left(E_{2}, E_{3}\right)$ then $P_{2} \circ P_{1} \in \mathcal{P}\left(E_{1}, E_{3}\right)$.

We call analytic function from a Banach space $E$ into a Banach space $F$ any function $f$ such that there exists $R>0$ (the radius of convergence), $A_{0} \in F$ and a sequence $\left(A_{k}\right)_{k \geq 1}$ with $A_{k} \in \mathcal{L}_{k}\left(E^{k}, F\right)$ satisfying:

$$
\sum_{k \geq 1}|\lambda|^{k}\left\|A_{k}\right\|_{\mathcal{L}_{k}\left(E^{k}, F\right)}<+\infty, \quad \forall \lambda \in \mathbf{R},|\lambda|<R
$$


and

$$
f(e):=A_{0}+\sum_{k \geq 1}\left\langle A_{k}, e, \ldots, e\right\rangle, \quad \forall e \in E,\|e\|_{E}<R
$$

We refer to the book $[4, \S 4]$, for further details on analytic functions in Banach spaces.

Acknowledgments: The author is more than grateful to Pr. J.F. Ganghoffer (with LEMTA, Nancy) for having him introduced to the theory of hyperelastic material.

\section{REFERENCES}

[1] F. Alouges, A. DeSimone, and A. Lefebvre. Optimal strokes for low Reynolds number swimmers: an example. J. Nonlinear Sci., 18(3):277-302, 2008.

[2] M. Boulakia. Existence of weak solutions for an interaction problem between an elastic structure and a compressible viscous fluid. J. Math. Pures Appl. (9), 84(11):1515-1554, 2005.

[3] M. Boulakia. Existence of weak solutions for the three-dimensional motion of an elastic structure in an incompressible fluid. J. Math. Fluid Mech., 9(2):262-294, 2007.

[4] H. Cartan. Théorie élémentaire des fonctions analytiques d'une ou plusieurs variables complexes. Avec le concours de Reiji Takahashi. Enseignement des Sciences. Hermann, Paris, 1961.

[5] A. Chambolle, B. Desjardins, M. J. Esteban, and C. Grandmont. Existence of weak solutions for the unsteady interaction of a viscous fluid with an elastic plate. J. Math. Fluid Mech., 7(3):368-404, 2005.

[6] T. Chambrion and A. Munnier. Generalized scallop theorem for linear swimmers. http://arxiv.org/abs/ 1008.1098, 2010.

[7] T. Chambrion and A. Munnier. On the locomotion and control of a self-propelled shape-changing body in a fluid. http://hal.archives-ouvertes.fr/hal-00422429/fr/, 2010.

[8] C. H. A. Cheng, D. Coutand, and S. Shkoller. Navier-Stokes equations interacting with a nonlinear elastic biofluid shell. SIAM J. Math. Anal., 39(3):742-800 (electronic), 2007.

[9] A. Cherman, J. Delgado, F. Duda, K. Ehlers, J. Koiller, and R. Montgomery. Low Reynolds number swimming in two dimensions. In Hamiltonian systems and celestial mechanics (Pátzcuaro, 1998), volume 6 of World Sci. Monogr. Ser. Math., pages 32-62. World Sci. Publ., River Edge, NJ, 2000.

[10] D. Coutand and S. Shkoller. Motion of an elastic solid inside an incompressible viscous fluid. Arch. Ration. Mech. Anal., 176(1):25-102, 2005.

[11] J. l. R. D'Alembert. Essai d'une nouvelle théorie de la résistance des fluides. Paris, 1752.

$[12]$ G. P. Galdi. On the steady, translational self-propelled motion of a symmetric body in a Navier-Stokes fluid. In Classical problems in mechanics, volume 1 of Quad. Mat., pages 97-169. Dept. Math., Seconda Univ. Napoli, Caserta, 1997.

[13] G. P. Galdi. On the steady self-propelled motion of a body in a viscous incompressible fluid. Arch. Ration. Mech. Anal., 148(1):53-88, 1999.

[14] E. Kanso and J. Marsden. Optimal motion of an articulated body in a perfect fluid. In in IEEE Conference on Decision and Control, pages 2511-2516, 2005.

[15] E. Kanso, J. E. Marsden, C. W. Rowley, and J. B. Melli-Huber. Locomotion of articulated bodies in a perfect fluid. J. Nonlinear Sci., 15(4):255-289, 2005.

[16] E. Kanso and P. K. Newton. Passive locomotion via normal-mode coupling in a submerged spring-mass system. J. Fluid Mech., 641:205-215, 2009.

[17] H. Lamb. Hydrodynamics. Cambridge Mathematical Library. Cambridge University Press, Cambridge, sixth edition, 1993.

[18] J. E. Marsden and T. J. R. Hughes. Mathematical foundations of elasticity. Dover Publications Inc., New York, 1994

[19] J. E. Marsden and T. S. Ratiu. Introduction to mechanics and symmetry, volume 17 of Texts in Applied Mathematics. Springer-Verlag, New York, second edition, 1999.

[20] J. B. Melli, C. W. Rowley, and D. S. Rufat. Motion planning for an articulated body in a perfect planar fluid. SIAM J. Appl. Dyn. Syst., 5(4):650-669 (electronic), 2006.

[21] A. Munnier. On the self-displacement of deformable bodies in a potential fluid flow. Math. Models Methods Appl. Sci., 18(11):1945-1981, december 2008. 
[22] A. Munnier. Locomotion of deformable bodies in an ideal fluid: Newtonian versus lagrangian formalism. $J$. Nonlinear Sci., 19(6):665-715, 2009.

[23] A. Munnier and B. Pinçon. Locomotion of articulated bodies in an ideal fluid: $2 \mathrm{~d}$ model with buoyancy, circulation and collisions. Math. Models Methods Appl. Sci., 2010.

[24] E. M. Purcell. Life at low reynolds number. American Journal of Physics, 45(1):3-11, 1977.

[25] S. Ross. Optimal flapping strokes for self-propulsion in a perfect fluid. In American Control Conference, 2006, pages 4118-4122, June 2006

[26] J. San Martín, J.-F. Scheid, T. Takahashi, and M. Tucsnak. An initial and boundary value problem modeling of fish-like swimming. Arch. Ration. Mech. Anal., 188(3):429-455, 2008.

[27] A. Shapere and F. Wilczek. Geometry of self-propulsion at low Reynolds number. J. Fluid Mech., 198:557-585, 1989. 